\title{
Search for Supersymmetry in the Dilepton Final State with Taus at CDF Run II \\ By
}

RoBert DAVID ForRest

B.S. (University of California, San Diego) 2001

\section{DISSERTATION}

Submitted in partial satisfaction of the requirements for the degree of

DOCTOR OF PHILOSOPHY

in

Physics

in the

OFFICE OF GRADUATE STUDIES

of the

UNIVERSITY OF CALIFORNIA

DAVIS

Approved:

Maxwell Chertok, Chair

John Conway

Mani Tripathi

Committee in Charge

2011 
Robert David Forrest

September 2011

Physics

\title{
Search for Supersymmetry in the Dilepton Final State with Taus at CDF Run II
}

\begin{abstract}
$\underline{\text { Abstract }}$
This thesis presents the results a search for chargino and neutralino supersymmetric particles yielding same signed dilepton final states including one hadronically decaying tau lepton using $6.0 \mathrm{fb}^{-1}$ of data collected by the the CDF II detector. This signature is important in SUSY models where, at high $\tan \beta$, the branching ratio of charginos and neutralinos to tau leptons becomes dominant. We study event acceptance, lepton identification cuts, and efficiencies. We set limits on the production cross section as a function of SUSY particle mass for certain generic models.
\end{abstract}




\section{Acknowledgements}

I owe deep gratitude to countless numbers of people that helped me get to this point. I truly could not have done it alone. Some require specific mention.

Maxwell Chertok, my advisor, has been a wonderful mentor and guided me through the melee for the past seven years. Many advisors leave students to fend for themselves, yet despite me being 1800 miles away, he has always been genuinely willing to help. I'm quite grateful. Robin Erbacher has been a mentor in many, many ways. I cut my teeth on her XFT project, but the lessons learned extended beyond Physics, like the fact that there is time to sleep when we die. Also, John Conway was a great influence, and I am definitely indebted to his support over the years. Mani Tripathi is hard to forget, and has taught me about the more human side of the profession.

A number of post-docs at Fermilab have been very helpful, some becoming friends for life in the process. Aron Soha, from my very first day in the midwest, has been extremely generous both professionally and personally. Alison Lister has been a great friend, educator, and an entertaining pro-European activist. Conversations with Tom Schwarz are always entertaining, mostly because we are on the same wavelength. It's refreshing to see someone who can have intelligent conversations outside of Physics; balance work, and still get Tollestrup Award for outstanding research. Andrew Ivanov was very helpful and tolerated me as I was learning the ropes on the XFT project. Cristobal Cuenca Almenar's energy and humor is hard to forget and much of the tau work in this analysis was based on his research. Sourabh Dube and Tom Wright have selflessly helped me with the trilepton analysis, I could not have blessed that analysis without them. For my thesis project Anton Anastassov and Shalhout Shalhout have helped tremendously as I encountered emergencies and had to learn new techniques on day time scales for the project to be a success.

The students around me have kept me going, it's refreshing to have friends that can sympathize with the life of a peer. Carley Kopecky is one of the kindest people I know, and it's been great having her as a friend. I could not have passed the prelim exam without studying with Emily Ashbaugh every day, for two months. Damien Martin is very knowledgeable, and conversations with him are always unique and fun. Will Johnson and Sho Maruyama are great, smart people to spend time with. Charles and David Cox have also been quite helpful at Fermilab. 
The people associated with Fermilab have been absolutely wonderful. All the QLC folks, especially Steve and Dee Hahn are the core of the CDF family, I am deeply appreciative, they are what make CDF what it is. JJ Schmidt is another wonderful guy, I could never have run a marathon without his coaching. Ankush Mitra tolerated me in his office, and became a good friend. Farrukh Azfar is a great cook and a great person. I also thank Dave Toback for genuinely caring about my success and diligently keeping me on track.

I also would like to thank the people that started my interest in the policy world. The URA and the UEC sent me to DC for three years straight. Everyone on these trips were quite influential to me, especially Herman White, who is great to have conversations with. Also, Anne Harrington and Frutuoso Santana became good friends in Chicago.

Thank you to Chicago, doubtless one of the best cities in the world, for teaching a Californian what weather is. I think I get it now. I have made several new friends during my time in Chicago. Dan Duggan and Tyler Dorland are great guys. Mike Backfish is one of the highest quality people I know. Bjoern Penning, Marcelle Soares-Santos, and Elisabetta Pianori are friends that formed the core of my Chicago experience. My roommates Ken Eatmon, Justin Brink and Jack Silverstein have made my time in Chicago unforgettable. Also Natalie Hoover and Samir El Rashidy along with Tim Lugo and Kristina Shih have been wonderful friends in the city. Angela Rossman has been terrific, and can somehow make the drive from Wisconsin, whenever needed, in record time.

I also want to thank my long-time friends, who have stuck with me despite my falling off the radar at times. Thanks to the DYU guys: Mike Romain, Julian Millikan, Mike Hanowsky and András Nady for keeping me human. Mike Weaver and Matt Osborne-Smith will always be close friends, even if I neglect to stay in touch for far too long. Suzi Riley is a marvelous friend and has not written me off despite my lack of communication; don't worry, I haven't forgotten you.

My girlfriend Bekah has been my rock for several years now, I am extremely lucky to have her by my side.

Finally, I'd like to thank my family: my sister Laura and my parents Barbara and Ray. You have my deepest gratitude, I couldn't have done it without you. 


\section{Contents}

1 Introduction 1

2 The Standard Model 4

2.1 Introduction . . . . . . . . . . . . . . . . . 4

2.2 Quantum Electrodynamics . . . . . . . . . . . . . . 7

2.3 Quantum Chromodynamics ............... 8

2.4 Electroweak Theory . . . . . . . . . . . . . . . . . 10

2.4.1 The Higgs Mechanism . . . . . . . . . . . . . . . . . . . 12

2.5 Issues with the Standard Model . . . . . . . . . . . . . . . 13

2.6 Supersymmetry . . . . . . . . . . . . . . . . . 15

2.6.1 Supersymmetry Theory . . . . . . . . . . . . . . 16

2.6.2 Supersymmetry Breaking . . . . . . . . . . . . . 18

2.6.3 R-Parity and the LSP . . . . . . . . . . . . . . 19

2.6.4 Production of Chargino and Neutralinos . . . . . . . . . . 19

2.6.5 Minimal Super Gravity . . . . . . . . . . . . . . . 20

2.6.6 Gauge Mediated Supersymmetry Breaking . . . . . . . . . . . 21

2.6.7 Simplified Models . . . . . . . . . . . . . . . . . . . 22

2.6.7.1 Simplified Gravity Model . . . . . . . . . . . 24

2.6.7.2 Simplified Gauge Model . . . . . . . . . . . . 25

3 Experimental Setup $\quad 26$

3.1 The Accelerator Chain . . . . . . . . . . . . . . 26 
3.1.1 Pre-Acceleration: The Linac, Booster and Main Injector . . 27

3.1.2 Antiproton Production and Storage . . . . . . . . . . . . . 28

3.1.3 The Tevatron . . . . . . . . . . . . . . . . . . . 29

3.1.3.1 Luminosity and Cross Section . . . . . . . . 29

3.2 The CDF II Detector . . . . . . . . . . . . . . . . . . . . . 31

3.2.1 The Tracking System: Silicon and the COT . . . . . . . . . . 32

3.2.2 The Calorimeters . . . . . . . . . . . . . . . . . 33

3.2.3 The Muon System . . . . . . . . . . . . . . . . 35

3.2.4 Luminosity Measurement . . . . . . . . . . . . . . . 35

3.2 .5 Triggering and $\mathrm{DAQ} \ldots \ldots \ldots . \ldots . \ldots . \ldots 37$

3.2.5.1 Level 1 . . . . . . . . . . . . . . . . . . 37

3.2.5.2 Level $2 \ldots \ldots . \ldots . \ldots . \ldots 40$

3.2.5.3 Level 3 . . . . . . . . . . . . . . . . . . . . 40

4 From Data to Physics $\quad 42$

4.1 Data and Monte Carlo Used . . . . . . . . . . . . . . . . . 43

4.2 Trigger Paths . . . . . . . . . . . . . . . . . 43

4.2.0.4 Level 2 Trigger Requirements . . . . . . . . . 45

4.2.0.5 Level 3 Trigger Requirements . . . . . . . . . . . 46

4.2.1 Trigger Efficiency Measurement . . . . . . . . . . . . . 46

4.2.2 Level 2 Efficiency Measurements . . . . . . . . . . . . . . . . . 47

4.2.3 Level 3 Efficiency Measurements . . . . . . . . . . . . . 52

4.2.3.1 Note on Electron Trigger Issue . . . . . . . . . 56

4.3 Lepton Selection . . . . . . . . . . . . . . . 56

4.3.1 Electron Identification . . . . . . . . . . . . . 56

4.3 .2 Muon Identification . . . . . . . . . . . . . . . . . . . 58

4.3.3 Tau Definition . . . . . . . . . . . . . . . 60

4.3.3.1 Tau Quantities ................ 61

4.3.3.2 Tau Scale Factors and Systematics . . . . . . . 63 
4.3.3.3 Tau Energy Scale . . . . . . . . . . . . . . . . 65

4.3 .4 Jet Definition . . . . . . . . . . . . . . . . . 65

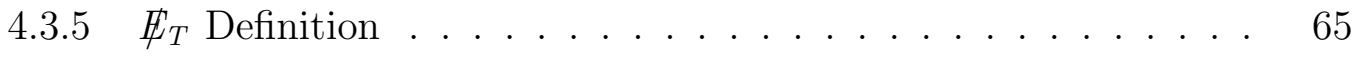

4.3 .6 Cosmic Veto . . . . . . . . . . . . . . . . 66

$\begin{array}{lll}5 & \text { Background Model } & 67\end{array}$

5.1 Monte Carlo Backgrounds . . . . . . . . . . . . . . . . . 68

5.2 Tau Fake Rate Backgrounds . . . . . . . . . . . . . . . . 68

5.2.1 Jet-to- $\tau$ Fake Rate Measurement . . . . . . . . . . . . 70

5.2.2 Jet-to- $\tau$ Fake Rate Verification . . . . . . . . . . . . . 71

5.2 .3 Jet-to- $\tau$ Fake Rate Application _. . . . . . . . . . . 73

5.2.4 Jet-to- $\tau$ Fake Rate Conversion Corrections . . . . . . . . . 79

6 Opposite-Signed Control Region $\quad 81$

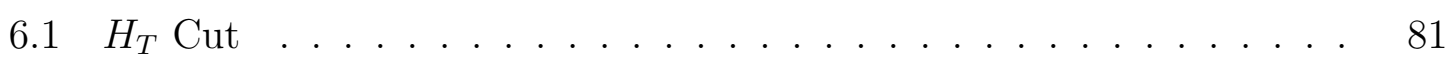

6.2 Opposite-Signed Results . . . . . . . . . . . . . . . . . . 82

$\begin{array}{lll}7 & \text { Systematic Uncertainties } & 87\end{array}$

7.1 Trigger Uncertainty . . . . . . . . . . . . . . . . . . . 87

7.2 Reconstruction and ID Uncertainty . . . . . . . . . . . 88

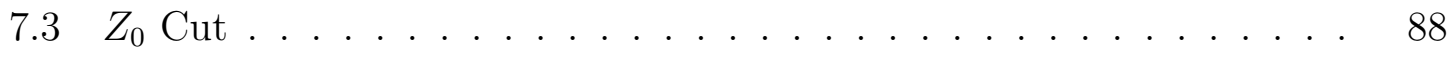

7.4 Standard Model Cross Sections . . . . . . . . . . . . . 88

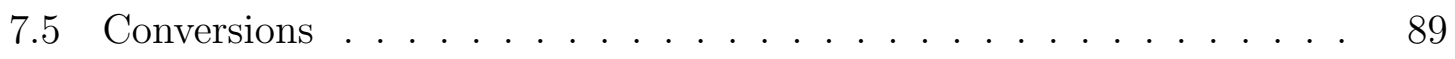

7.6 Luminosity . . . . . . . . . . . . . . . . . . . . . 89

7.7 Jet $\rightarrow \tau$ Fake Rate $\ldots \ldots \ldots \ldots \ldots \ldots \ldots$

7.8 Jet Energy Scale $\ldots \ldots \ldots \ldots$

7.9 Tau Energy Scale . . . . . . . . . . . . . . . . . . . . . . 89

$\begin{array}{lll}8 & \text { Results } & 91\end{array}$

8.1 Same Signed Data Results . . . . . . . . . . . . . . . . . . 91 
8.2 Reserved Signal Sub-Regions . . . . . . . . . . . . . . . . . . . . . 97

8.2.1 $M(\tau, \mathrm{l})>200 \mathrm{GeV} / c^{2} \ldots \ldots \ldots 9 . \ldots \ldots$

$8.2 .2 \quad H_{t}>300 \mathrm{GeV} / c \ldots \ldots . \ldots \ldots 7$

8.2.3 $P_{t}(\tau)>100 \mathrm{GeV} / c$ and $P_{t}(l)>100 \mathrm{GeV} / c \ldots . . . . . .97$

8.3 Observed Limits . . . . . . . . . . . . . . . . . . . . . . . . . . 100

8.3.1 $\mathbb{E}_{T}$ Cut Optimization ................. 100

8.3.2 Limit Contours . . . . . . . . . . . . . . . . . . . 102

9 Conclusions $\quad 106$

$\begin{array}{ll}\text { Bibliography } & 108\end{array}$

$\begin{array}{ll}\text { List of Figures } & 112\end{array}$

List of Tables $\quad 116$

$\begin{array}{ll}\text { Glossary } & 118\end{array}$ 


\section{Chapter 1}

\section{Introduction}

The standard model (SM) of Particle Physics is an astounding accomplishment. It accurately describes the interactions of the most fundamental particles of nature and, over the last century, has survived the test of a countless number of experiments. Intrinsically, the standard model is a quantum field theory consisting of $\mathrm{SU}(3)_{C} \times \mathrm{SU}(2)_{L} \times \mathrm{U}(1)_{Y}$ gauge groups wherein particles and forces emerge as resonances of the fields. The theories of the strong, weak and electromagnetic forces are consistently encompassed in their respective gauge groups. Particles emerge from the theory in two types; fermions constitute stable matter, while the exchange of bosons mediates the forces between the particles.

A generation of particle physicists have been tasked with faithfully proving time and time again the validity of the standard model. But despite its success, the maddening truth is that we know it is flawed. Among other issues, at large energies, the equations predicting cross sections diverge and must be artificially renormalized. It is also an incomplete theory, not accounting for the force of gravity, or the existence of Dark Matter, or of Dark Energy.

Supersymmetry (SUSY) is an elegant proposed theory that rectifies a number of problems with the standard model. It adds a symmetry to the standard model fermion and boson states, and effectively doubles the number of particles that would exist. For every standard model boson, there is a predicted SUSY fermion and vice-versa. If nature is supersymmetric, it would beautifully solve some of the basic problems underlying the standard model. Unfortunately, since we have not observed SUSY particles, if Supersymmetry exists it must be a broken symmetry wherein the masses 
of the SUSY partner particles are significantly higher than their standard model counterparts. The purpose of this dissertation is to detect or disprove the existence of these high mass SUSY particles.

Controlled creation of high mass particles in experiment necessitates very high energy particle collisions. The energy of the colliding particles provides the phase space for creating SUSY particles. Fermilab's Tevatron is a $6.3 \mathrm{~km}$ circumference accelerator that collides protons and antiprotons at a center of mass energy of $\sqrt{s}=$ $1.96 \mathrm{TeV}$ at two points along its circumference. Two large particle detectors, CDF and $\mathrm{D} \varnothing$, are placed at the point of interactions to measure and reconstruct events emerging from the collisions.

The analysis presented in this dissertation is a search for the production of a specific type of SUSY particles, the so-called charginos and neutralinos. Although SUSY theories can have many forms, several share a commonality in the existence and characteristic decay of the charginos and neutralinos. SUSY particles decay almost immediately after production in a cascading chain ending with the standard model particles we measure with the CDF detector. Three leptons are the only detectable particles left after the decay, although we can use non-detected particles as a characteristic missing energy signal as well. This analysis is designed to look for two of the three leptons having the same sign electric charge while requiring one of the leptons to be a tau. The choice of looking for two of the three leptons increases our detector acceptance, and choosing the same signed lepton pair dramatically decreases standard model backgrounds. Including a tau lepton increases our sensitivity to unique SUSY theory parameter space that has not already been excluded by experiment.

No SUSY signal is found over the background of the standard model, so we place 95\% confidence level upper limits on the possible production cross sections of generic SUSY models. These limits can then be translated by theorists into specific SUSY variants, and serve to exclude possible model parameter space from such theories.

In Chapter 2 I describe the standard model and its imperfections, as well as the beauty of SUSY and how it might solve some of the standard model shortcomings. Chapter 3 is a description of the experimental setup, from the accelerator chain, to the Tevatron, to the CDF detector. Chapter 4 describes how the raw data are translated into real physical objects. Our simulated detector response to the standard model is described in Chapter 5 as well as event cuts used to improve our sensitivity to SUSY. I then validate and cross check the model in Chapter 6 before examining the 
systematic errors in Chapter 7 then looking at the blinded data in the signal region and extracting the results in Chapter 8. Final conclusions are presented in Chapter 9. 


\section{Chapter 2}

\section{The Standard Model}

\subsection{Introduction}

The standard model is the crowning achievement of 20th century particle physics. It emerged in the late 1970's as an encapsulation of several previously developed theories. It unites three forces, the strong, weak and electromagnetic force as well as their respective particles into one coherent field theory. Countless experiments have validated its predictions [1]. It has been such a success that for the last 20 years the field has primarily concerned itself with discovering the final remaining piece of the standard model, the Higgs boson.

Generally, the particles of the standard model are divided into fermions and bosons. The bosons are responsible primarily for mediating the forces, they interact with the fermions which make up most of the stable matter of the universe [2]. For a listing of the fermions, see Table 2.1 and for the bosons, see Table 2.2.

\begin{tabular}{|l|c|l|l|l|}
\hline \hline & Charge & First Generation & Second Generation & Third Generation \\
\hline Leptons & -1 & Electron $(\boldsymbol{e})$ & Muon $(\boldsymbol{\mu})$ & Tau $(\boldsymbol{\tau})$ \\
& 0 & Electron neutrino $\left(\boldsymbol{\nu}_{\boldsymbol{e}}\right)$ & Muon neutrino $\left(\boldsymbol{\nu}_{\boldsymbol{\mu}}\right)$ & Tau neutrino $\left(\boldsymbol{\nu}_{\boldsymbol{\tau}}\right)$ \\
\hline \hline Quarks & $+\frac{2}{3}$ & $\operatorname{Up}(\boldsymbol{u})$ & Charm $(\boldsymbol{c})$ & Top $(\boldsymbol{t})$ \\
& $-\frac{1}{3}$ & Down $(\boldsymbol{d})$ & Strange $(\boldsymbol{s})$ & Bottom $(\boldsymbol{b})$ \\
\hline \hline
\end{tabular}

Table 2.1: The fermions of the standard model ( $\operatorname{spin} \frac{1}{2}$, antiparticles have opposite charge. 


\begin{tabular}{|l|c|l|}
\hline \hline Mediator & Charge & Force \\
\hline Gluon $(\boldsymbol{g})$ & 0 & Strong \\
\hline Photon $(\boldsymbol{\gamma})$ & 0 & Electromagnetic \\
\hline $\boldsymbol{W}^{ \pm}$ & \pm 1 & Weak (charged) \\
\hline $\boldsymbol{Z}^{\mathbf{0}}$ & 0 & Weak (neutral) \\
\hline \hline
\end{tabular}

Table 2.2: The bosonic force mediators of the standard model (spin 1).

Fermions are divided into two types: quarks and leptons, each of which contains three generations of particle pairs. Every fermion also contains an antiparticle, quarks form tightly formed constituent particles bound in pairs or triplets by the strong force. Gluons are the boson force mediators that bind these constituent particles. So for example, a proton is a collection of two up and one down type quarks (uud), tightly bound by a sea of gluons.

Leptons are the other type of fermion; each of the three generations of leptons contains a massive charged particle as well as a very light and neutral neutrino. The leptons interact with the $W^{ \pm}$and $Z^{0}$ bosons which carry the weak force [3]. The electron $\left(e^{-}\right)$for example, is the first generation lepton, and is associated with the electron neutrino $\nu_{e}$. The electromagnetic force is mediated by the photon, $\gamma$, and interacts with all charged particles.

It is important to note that the theories in the standard model are mathematically represented by symmetry groups. The strong force is a $\mathrm{SU}(3)_{C}$ color symmetric theory invariant under local gauge transformations. The electroweak theory is an $\mathrm{SU}(2)_{L}$ represented group that contains a so-called weak-isospin symmetry, as well as a hypercharge symmetry component $\mathrm{U}(1)_{Y}$. The combination of the three symmetries reflect the symmetry of the whole standard model, $\mathrm{SU}(3)_{C} \times \mathrm{SU}(2)_{L} \times \mathrm{U}(1)_{Y}$.

What follows is a more detailed description of the forces and particles of the standard model, how they fit into these groups, and why we need a Higgs boson. We will then look at some of the shortcomings of the standard model. 


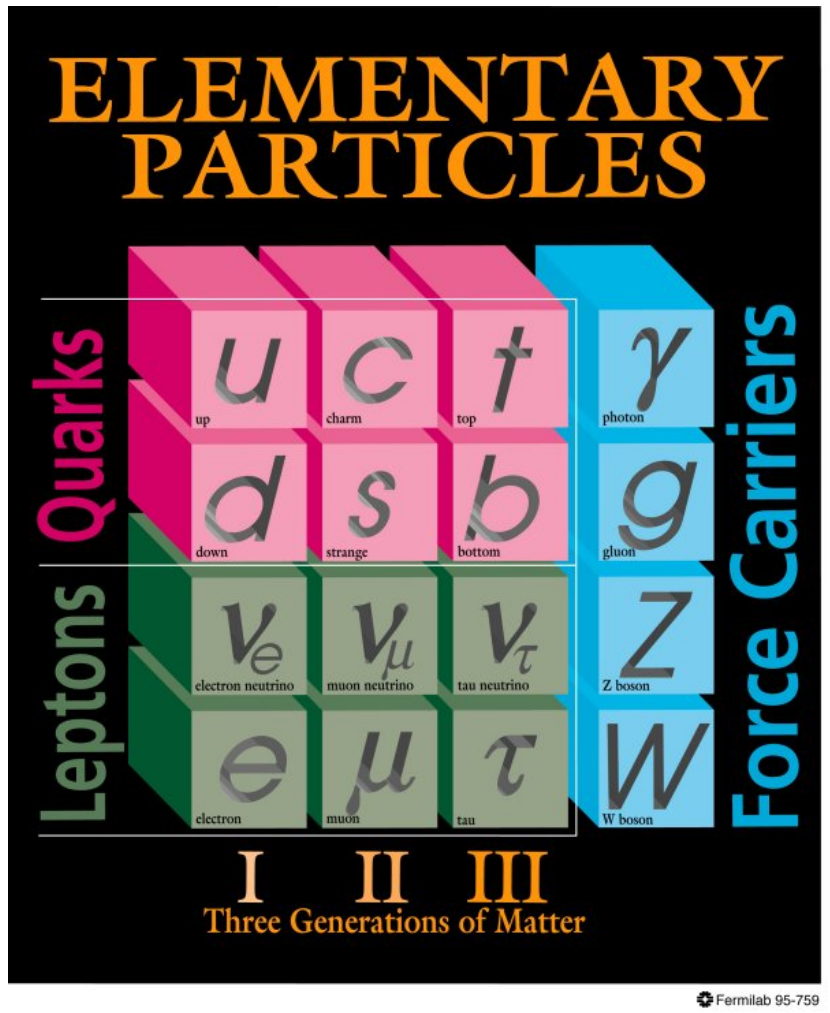

Figure 2.1: Elementary particles in the standard model. (Image courtesy of Fermilab Visual Media Services) 


\subsection{Quantum Electrodynamics}

We know through observation that we want to construct a theory that describes spin $\frac{1}{2}$ particles; the Schrodinger equation is one such example in Quantum Mechanics [4]. The relativistic, Lorentz-invariant version is the Dirac equation, whose Lagrangian takes the form:

$$
\mathcal{L}=\bar{\psi}\left(i \gamma^{\mu} \partial_{\mu}-m\right) \psi
$$

Where $\psi$ is the field, $m$ is the mass of the particle, and $\gamma^{\mu}$ are the Dirac matrices. We see the field theories of the standard model share a defining feature, that of local gauge invariance. That is, they are invariant under a continuous group of transformations called gauge transformations. If we have a field $\psi$ and we do some operation $\psi^{\prime}$ we get:

$$
\psi \rightarrow \psi^{\prime}=e^{i Q \theta} \psi
$$

Where the constant in front of $\psi$ is dependent on $Q \theta$. The $Q$ term will eventually be identified as a particle charge. $\theta$ is an arbitrary phase, and standard model equations, as a feature of gauge invariance, will generally contain terms with some form of $\bar{\psi} \psi$ serving as a location independent cancelation of the phase term. The example in equation (2.2) is the so called $\mathrm{U}(1)$ abelian gauge group.

In order to shift this global symmetry to a local one, dependent on position $(\theta \rightarrow \theta(x))$, the derivative in the Lagrangian $\left(\partial_{\mu}\right)$ must be replaced by the covariant derivative.

$$
D_{\mu} \equiv \partial_{\mu}-i e A_{\mu}
$$

where $A_{\mu}$ is a vector field (spin 1) that transforms like:

$$
A_{\mu} \rightarrow A_{\mu}^{\prime} \equiv A_{\mu}+\frac{1}{e} \partial_{\mu} \theta(x)
$$


After this replacement, the Lagrangian for the Dirac equation becomes

$$
\mathcal{L}=\bar{\psi}\left(i \gamma^{\mu} D_{\mu}-m\right) \psi=\bar{\psi}\left(i \gamma^{\mu} \partial_{\mu}-m\right) \psi+e A_{\mu}\left(\bar{\psi} \gamma^{\mu} \psi\right)
$$

Which maintains the desired local gauge invariance when we make the substitution in Equation 2.2. At this point we add a term to the above equation to include the kinematic energy of the new field. Fortunately, classical electromagnetism provides us with a perfectly acceptable and invariant term for the field strength:

$$
\mathcal{L}=-\frac{1}{4} F_{\mu \nu} F^{\mu \nu}
$$

where $\mathrm{F}$ is the electromagnetic field tensor, defined as:

$$
F_{\mu \nu} \equiv \partial_{\mu} A_{\nu}-\partial_{\nu} A_{\mu}
$$

So the total Lagrangian is now:

$$
\mathcal{L}=\bar{\psi}\left(i \gamma^{\mu} \partial_{\mu}-m\right) \psi-\frac{1}{4} F_{\mu \nu} F^{\mu \nu}+e Q A_{\mu}\left(\bar{\psi} \gamma^{\mu} \psi\right)
$$

equation (2.8) is the complete expression of QED. The first term is the original Dirac equation: a quantum mechanical wave equation for a single particle field of mass $m$. The second term is Maxwell's equation, and the third is an interaction term between an electromagnetic field and a particle of charge $e$. All of QED is a description of Feynman diagrams constructed with vertices resembling Figure 2.2.

\subsection{Quantum Chromodynamics}

Quantum ChromoDynamics (QCD) is the piece of the standard model describing the strong force, which is mediated by gluons carrying color charge [5]. QCD follows in the footsteps of QED using the same gauge invariant field description, with modifications specific to the observed behavior of strong interactions. Specifically it must account for the observation in QCD of asymptotic freedom, that the coupling con- 

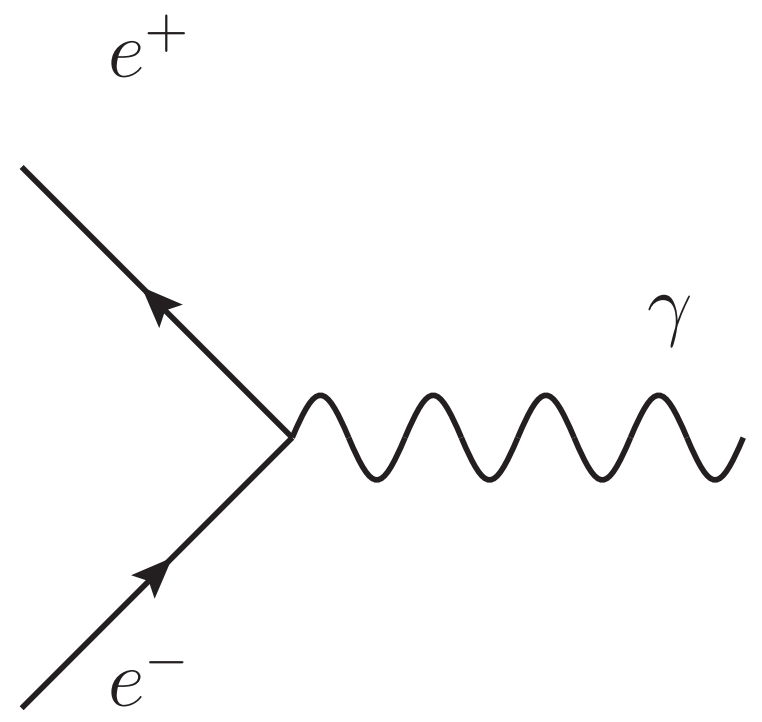

Figure 2.2: Fundamental Feynman Diagram for QED.

stant becomes weak at short distances as the momentum of interactions increases. This suggests that QCD use a non-Abelian gauge theory: a gauge theory with noncommuting local symmetry. We also know that, besides spin and flavor, quarks carry one of three color charges. This suggests the use of the symmetry group $\mathrm{SU}(3)$ to represent the gauge quantum numbers of the quarks in the color basis. If quarks are assigned the fundamental representation of the group $\mathrm{SU}(3)$, the quanta of the field are called gluons.

Under the SU(3) representation, it turns out, one can make our necessary invariant in two ways: the totally antisymmetric combination of three quark (or antiquark) colors, or the combination of a quark and antiquark:

$$
\epsilon^{i j k} q_{i} q_{j} q_{k}, \quad \epsilon_{i j k} q^{i} q^{j} q^{k}, \quad \bar{q}^{i} q_{i}
$$

These color neutral quark orderings in Equation 2.9 represent observable particles in nature. Baryons such as protons $(u u d)$ and neutrons $(d d u)$ as well as antibaryons contain three quarks or antiquarks. Mesons, such as the pion $(u \bar{d})$, contain a quark and antiquark.

To arrive at the Lagrangian, we repeat the process of QED in Section 2.2, starting 
with a Dirac equation and working with an $\mathrm{SU}(3)$ group. We apply a symmetry operation, and restore invariance by adding correction terms [6].

$$
\mathcal{L}=\sum_{\text {flavor }} \overline{q_{a}}\left(i \gamma^{\mu} D_{\mu}-m_{q}\right)_{a b} q_{b}-\frac{1}{4} F_{\alpha \beta}^{A} F_{A}^{\alpha \beta},
$$

which is very much like Equation 2.8 but we now have a sum running over all quark flavors. Again we have a term $F_{\alpha \beta}^{A}$ which is the field strength tensor of the gluon field $A_{\alpha}^{A}$ :

$$
F_{\alpha \beta}^{A}=\partial_{\alpha} A_{\beta}^{A}-\partial_{\beta} A_{\alpha}^{A}-g f^{A B C} A_{\alpha}^{B} A_{\beta}^{C},
$$

We see the sum $(\mathrm{A}, \mathrm{B}, \mathrm{C} \ldots)$ over the eight gluon degrees of freedom and observe the extra term in equation 2.11 compared to the QED equivalent as reflecting the nonAbelian description of QCD. The extra term has several consequences. First, it leads to gluon self interactions, unlike the photons of QED. Self-interactions imply that the coupling $(\mathrm{g})$ is small at large energies or short distances - asymptotic freedom. The coupling also becomes very large at small energies or long distances, an effect leading to the confinement of quarks into the color neutral groups of equation 2.9.

\subsection{Electroweak Theory}

The weak force emerges as a theory to explain flavor changing processes such as the experimental observation of neutron $\beta$ decay, the process $n \rightarrow p+e^{-}+\bar{\nu}_{e}$. While QED describes the transmission of electromagnetic force through electric charge, and QCD describes the transmission of strong force through color, neither of these theories can describe $\beta$ decay. Indeed neither QED nor QCD describe any mechanism wherein heavy particles change flavor and decay to lighter ones.

The solution, proposed by Glashow, Salam and Weinberg [7] was to combine the QED group with an $\mathrm{SU}(2)$ group according to constraints of experiment. The result is an $\mathrm{SU}(2)_{L} \otimes \mathrm{U}(1)_{\mathrm{Y}}$ theory called the Electroweak theory. Despite being unified, the discrepancy in strength between the Electromagnetic force and the Electroweak force arises from the inclusion of massive mediators of the weak force. But there are additional considerations stemming from behavioral differences between the two 
forces, namely the fact the weak charged force interacts only with left-handed helicity states of quarks and leptons. We note that the electromagnetic vertex factors are vectorial, yet the weak vertex factors are a mix of axial and vectorial. By placing what is essentially a projection operator into the particle spinor, for massless states, we end up with left handed fermions transforming as doublets and right handed fermions transforming as singlets. For example for electrons:

$$
\psi_{1}=\left(\begin{array}{c}
e \\
\nu_{e}
\end{array}\right)_{l} \quad \psi_{2}=e_{r}
$$

We follow the same prescription for the Lagrangian as in previous theories wherein the covariant derivative is defined now as:

$$
D_{\mu} \equiv \partial_{\mu}-i g \boldsymbol{T} \boldsymbol{W}_{\mu}-i g^{\prime} \frac{Y}{2} B_{\mu}
$$

Where $g$ and $g^{\prime}$ are coupling constants, $\mathrm{Y}$ is the $\mathrm{U}(1)$ charge, and $W_{\mu}$ and $B_{\mu}$ are the $\mathrm{SU}(2)$ and $\mathrm{U}(1)$ gauge bosons respectively [6]. The Lagrangian of the theory is:

$$
\mathcal{L}_{G}=\Sigma i \bar{\psi}_{j}(x) \gamma^{\mu} D_{\mu} \psi_{j}(x)-\frac{1}{4} W_{\mu \nu}^{i} W_{i}^{\mu \nu}-\frac{1}{4} B_{\mu \nu} B^{\mu \nu}
$$

Where the last two terms may be re expressed as:

$$
\begin{aligned}
W_{\mu}^{ \pm} & =\frac{1}{\sqrt{2}}\left(W_{\mu}^{1} \mp i W_{\mu}^{2}\right) \\
Z_{\mu} & =\cos \theta_{W} W_{\mu}^{3}-\sin \theta_{W} B_{\mu} \\
A_{\mu} & =\sin \theta_{W} W_{\mu}^{3}+\cos \theta_{W} B_{\mu}
\end{aligned}
$$

where $A_{\mu}$ is the photon field, which is now incorporated into the theory. $W_{\mu}^{ \pm}$and $Z_{\mu}$ are the gauge bosons that are the mediators of the weak force. The angle $\cos \theta_{W}$ is the so called weak mixing angle which is related to the couplings of the theory by $\tan \theta_{W}=g^{\prime} / g$.

We are left with four mediators: the photon emerges identically to QED, a neutral boson $Z$, and two charged bosons $W^{ \pm}$mediate of the weak force. We have managed to unite the weak and electromagnetic forces into one electroweak theory. 


\subsubsection{The Higgs Mechanism}

The problem with the bosons in section 2.4, is that these bosons are massless in the equations, and adding a mass term by hand would break gauge invariance. For them to act at very short distances and still have weak couplings, the mediators need mass. In the standard model, he Higgs mechanism is responsible for adding mass to the gauge fields while maintaining local gauge invariance [8]. To do this we break the local gauge symmetry of the $\mathrm{SU}(2)_{L}$ group.

If we consider a potential of the form:

$$
V(\Phi)=\mu^{2} \Phi^{\dagger} \Phi+\lambda\left(\Phi^{\dagger} \Phi\right)^{2}
$$

if $\mu^{2}<0$, it has two minima at :

$$
\pm \sqrt{-\frac{\mu^{2}}{2 \lambda}} \equiv \pm \frac{v}{\sqrt{2}}
$$

Choosing one of these minima breaks the $\mathrm{SU}(2) \mathrm{XU}(1)$ symmetry and the field acquires a vacuum expectation value (VEV).

$$
\langle 0|\Phi| 0\rangle \neq 0
$$

According to the Goldstone theorem fields such as 2.19 that have a VEV acquire an extra massless Goldstone boson. We can then transform away this field and essentially add them to the degrees of freedom of the Electroweak gauge particles. So our massless vector fields that originally had two degrees of transverse polarization acquire a mass, or a longitudinal polarization, from the degrees of freedom of the Goldstone boson. This Goldstone boson effectively disappears from the theory. This is the so-called Higgs mechanism, and when introduced into the standard model Lagrangian, we obtain mass relations for the fermions through Yukawa couplings, the massive gauge bosons $\left(W^{ \pm}, Z\right)$, and a new Higgs boson $(H)$. 


\subsection{Issues with the Standard Model}

The standard model has had proved enormously successful, indeed it has been experimentally verified repeatedly throughout the years. The experiment UA1 discovered the massive vector bosons of the Electroweak theory $[9,10]$ described in section 2.4. The TeVatron discovered the last predicted quark, the top quark $(t)$. And both the Large Hadron Collider and the TeVatron are quickly closing in on the Higgs boson's expected mass range. If the Higgs is discovered, the standard model will be validated and completely observed by experiment.

Unfortunately, we know the standard model is not a complete description of nature and is deficient in many ways.

Firstly, and most obviously, the standard model is incomplete in that it does not attempt to describe gravity. It is assumed gravity is far too weak to couple with the other three forces directly so we look at the standard model as an effective theory describing low energies.

Then there are some unsettling questions about the theory itself. The reasoning behind the three generational structure of the quarks and leptons is not explained. The arbitrary free parameters of the theory such as charge, masses and couplings need to be measured as well, and are not predicted by the theory. We have also recently discovered that neutrinos oscillate between different flavors implying they have a finite but very small mass [11], something the standard model does not predict and must be patched to accommodate. We also have an observed matter-antimatter asymmetry in the universe that is wholly unaccounted for. Recent Cosmic Microwave Background observations indicate the universe's expansion is accelerating, indicating dark energy makes up a vast majority $\approx 73 \%$ of the energy density of the Universe [12]; the standard model gives no indication of what this might be.

Through many observations of gravitational effects, we know the universe has an abundance of Dark Matter, that is, matter that only interacts gravitationally through its mass term or weakly through the weak force[13]. Dark matter clusters in galaxies and accounts for about $23 \%$ of the mass-energy density of the Universe. The standard model does not give a Dark Matter candidate.

Probably the most notable issue with the standard model is the fine-tuning problem wherein some of the parameters of the standard model need to be very precisely 
adjusted in order to agree with their measured values. Scalar fields like the Higgs receive corrections to their masses through radiative corrections. That is to say, high order loop corrections to the Higgs mass are required to be finite:

$$
\Delta m_{H}^{2} \propto \int^{\Lambda} f(k, \text { external lines }) d^{4} k=\text { finite. }
$$

Where $\Lambda$ goes all the way to a cutoff at the plank scale $\Lambda 10^{19} \mathrm{GeV}$ where gravity becomes important. Normally fermions and gauge bosons are protected from divergence by their intrinsic symmetries. The scalar Higgs however, is not. Therefore one loop diagrams due to fermions like Figure 2.3 as well as similar effects due to bosons contribute to the Higgs mass correction like:

$$
\int^{\Lambda} \frac{1}{k^{2}-M_{H}^{2}} d^{4} k
$$

which diverges.

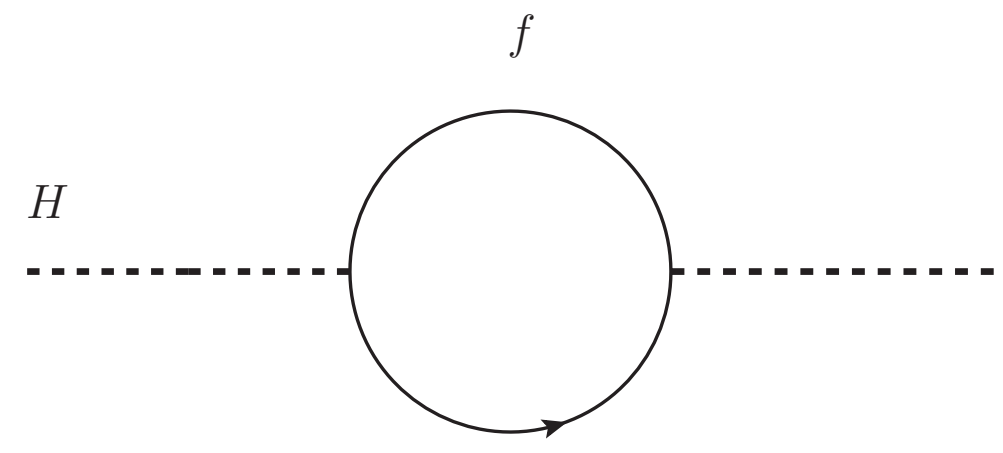

Figure 2.3: One loop corrections to the Higgs Mass due to a Dirac fermion.

The total corrections to the Higgs mass would take the form

$$
m_{\text {phys }}^{2}=m^{2}+\lambda^{2} \Lambda^{2}
$$

Where $m^{2}$ is the bare mass, $m_{\text {phys }}^{2}$ is the observed mass, which we know to be on the order of the VEV of the Higgs $v \approx 246 \mathrm{GeV}$. The value of $\lambda$ must be close to unity since we need perturbation to work. $\Lambda^{2}$ contains very large corrections from every 
particle that couples to the Higgs. The large value of $\Lambda^{2}$ must be almost exactly, but not quite completely, canceled by the bare mass term $m^{2}$ to give the expected result, a cancelation of over 30 orders of magnitude. This is so unnatural and fine tuned, it is called the hierarchy problem [14].

\subsection{Supersymmetry}

We saw in section 2.5 and equation 2.21 that the Higgs receives corrections from couplings like the diagrams shown in Figure 2.3. Specifically, the one loop correction from a fermion with a coupling in the Lagrangian like $-\lambda_{f} H \bar{f} f$ is:

$$
\Delta m_{H}^{2}=-\frac{\left|\lambda_{f}^{2}\right|}{8 \pi^{2}} \Lambda^{2}+\ldots
$$

with higher order contributions entering from other diagrams. A scalar loop, with coupling $-\lambda_{s}|H|^{2}|s|^{2}$ would enter through a loop diagram such as that in Figure 2.4 with a correction :

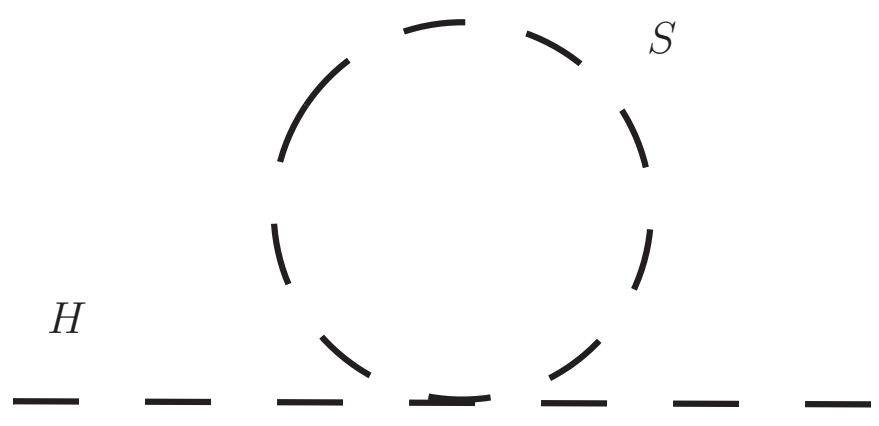

Figure 2.4: One loop scalar corrections to the Higgs Mass.

$$
\Delta m_{H}^{2}=\frac{\left|\lambda_{s}\right|}{16 \pi^{2}} \Lambda^{2}+\ldots
$$

The correction term is enticingly close to what we need to cancel out the large $\Lambda$ divergences in the expression for $\Delta m_{H}^{2}$ due to the relative minus sign. This is true for most of the higher order terms in the expression as well. If we were to have boson 
loops to systematically cancel out the divergences of the fermion loops term for term, we would have a natural solution to the large divergences and thus the hierarchy problem [15].

\subsubsection{Supersymmetry Theory}

The theory of Supersymmetry (SUSY) introduces an operator which relates fermions and bosons $[1,14]$. That is, there exists a transformation that turns bosons into fermions and vice-versa:

$$
\left.\left.Q \mid \text { Boson }\rangle=\mid \text { Fermion }\rangle \quad Q^{\dagger} \mid \text { Fermion }\right\rangle=\mid \text { Boson }\right\rangle
$$

Both $Q$ and $Q^{\dagger}$ are fermionic operators that carry spin $1 / 2$ so the symmetry they represent is a space-time symmetry. As a matter of fact, the SUSY symmetry generators represent the only other remaining symmetry of the Lorentz group.

The generators $Q$ and $Q^{\dagger}$ commute with the generators of the gauge transformations of the standard model implying they exist in the same representation of the gauge group. That is to say, the superpartners must have the same quantum numbers (charge, isospin, ...) as their standard model counterparts.

So for each chiral fermion, or fermions whose right and left handed components transform differently, we now have a bosonic counterpart. For each of the gauge bosons in the standard model we have a fermionic gaugino counterpart with spin differing by $1 / 2$ a unit. The standard model particles and their SUSY counterparts must be in the same super-multiplet.

We prepend an 's' in front of the super-partners partners of the standard model fermions to denote they are scalars, we add an '-ino' to the end of the super-partners of the standard model bosons. A tilde $\left(^{\sim}\right)$ is added to the symbol to denote the SUSY counterpart. So for example, the super-partner of the left-handed electron is called the selectron and denoted $\widetilde{e}_{L}$, although it is a spin-0 scalar and has no helicity.

We now need to organize our new particles into appropriate chiral or gauge supermultiplets. The chiral super-multiplets are shown in Table 2.3 and the gauge supermultiplets are shown in Table 2.4 . 


\begin{tabular}{|c|c|c|c|}
\hline \multicolumn{2}{|l|}{ Names } & Spin 1/2 & Spin 0 \\
\hline \multirow{5}{*}{$\begin{array}{c}\text { squarks, } \\
\text { quarks } \\
(\times 3 \text { families }) \\
\text { sleptons } \\
\text { leptons } \\
(\times 3 \text { families })\end{array}$} & $\hat{Q}$ & $\left(u_{L} d_{L}\right)$ & $\left(\tilde{u}_{L} \tilde{d}_{L}\right)$ \\
\hline & $\hat{U}$ & $u_{R}^{\dagger}$ & $\tilde{u}_{R}^{*}$ \\
\hline & $\hat{D}$ & $d_{R}^{\dagger}$ & $\tilde{d}_{R}^{*}$ \\
\hline & $\hat{L}$ & $\left(\nu e_{L}\right)$ & $\left(\tilde{\nu} \tilde{e}_{L}\right)$ \\
\hline & $\hat{E}$ & $e_{R}^{\dagger}$ & $\tilde{e}_{R}^{*}$ \\
\hline \multirow{2}{*}{$\begin{array}{l}\text { Higgs, } \\
\text { higgsinos }\end{array}$} & $\hat{H}_{u}$ & $\left(\tilde{H}_{u}^{+} \tilde{H}_{u}^{0}\right)$ & $\left(H_{u}^{+} H_{u}^{0}\right)$ \\
\hline & $\hat{H}_{d}$ & $\left(\tilde{H}_{d}^{0} \tilde{H}_{d}^{-}\right)$ & $\left(H_{d}^{0} H_{d}^{-}\right)$ \\
\hline
\end{tabular}

Table 2.3: Chiral supermultiplets of the MSSM.

\begin{tabular}{cc|c|c}
\hline \hline Names & & Spin 1/2 & Spin 1 \\
\hline \multirow{2}{*}{ EWK bosons } & $\hat{W}$ & $\tilde{W}^{ \pm} \tilde{W}^{0}$ & $W^{ \pm} W^{0}$ \\
& $\hat{B}$ & $B^{0}$ & $\tilde{B}^{0}$ \\
\cline { 2 - 4 } Strong bosons & $\hat{g}$ & $\tilde{g}$ & $g$ \\
\hline \hline
\end{tabular}

Table 2.4: Gauge supermultiplets of the MSSM.

The chiral super-multiplets containing the sfermions of SUSY in Table 2.3 contain separately the right and left handed components of the quarks and leptons. The Higgs with spin-0 also must reside in a chiral super-multiplet, but because of a gauge anomaly in quantum theory a single higgs would create, we must have two Higgs super-multiplets that essentially serve to cancel this anomaly. We end up with four higgs bosons and four higinos, a linear combination of $H_{d}^{0}$ and $H_{u}^{0}$ give us our standard model scalar higgs.

The gauge super-multiplets contain the vector bosons of the standard model and their super-partners. The SUSY fermions are called the gauginos and respect the same $S U(2)_{L} \times U(1)$ gauge symmetries of the standard model. The gluino $\widetilde{g}$ is the super-partner of the gluon and as expected, mediates the color gauge interactions of the squarks. The super-partners of the electroweak sector are the winos $(\widetilde{W} \pm, \widetilde{W})$ and bino $(\widetilde{B})$. If SUSY were unbroken, the neutral wino $(\widetilde{W})$ and bino $(\widetilde{B})$ would mix to form the photino $(\widetilde{\gamma})$ and zino $\left(\widetilde{Z}^{0}\right)$ mass eigenstates whose mass would mirror their standard model counterparts. 


\subsubsection{Supersymmetry Breaking}

We know we have yet to detect a SUSY particle in nature, so if SUSY exists it must be a broken symmetry in its natural state[16]. The SUSY breaking is not achieved by the SUSY term in the Lagrangian itself, such a thing would eliminate the cancelation between scalar and fermion terms in Equations 2.23 and 2.22. We assume then the breaking take place in a so called 'soft' term of the Lagrangian, $\mathcal{L}_{\text {soft }}$ so that

$$
\mathcal{L}=\mathcal{L}_{\mathrm{SUSY}}+\mathcal{L}_{\mathrm{soft}}
$$

So mass splittings between the standard model particles and their SUSY counterparts are wholly encapsulated in $\mathcal{L}_{\text {soft }}$ and this term will determine exactly how SUSY is broken.

An effect of the breaking of SUSY and the resultant mass splitting between the particles and the sparticles, is that the particles listed in Tables 2.3 and 2.4 may not ultimately be the mass eigenstates of the observed particles. There can be mixing between the electroweak gauginos and higgsinos, as well as within the squarks, sleptons and Higgs scalars. These will be the observables we will search for in our detector.

For example, when the electroweak gauginos and higgsinos mix, the mass eigenstates are linear combinations called charginos $\left(\tilde{\chi}^{ \pm}\right)$and neutralinos $\left(\tilde{\chi}^{0}\right)$. There are two charginos $\left(\widetilde{\chi}_{1,2}^{ \pm}\right)$and four neutralinos $\left(\widetilde{\chi}_{1,2,3,4}^{0}\right)$ ordered by increasing mass. The composition and behavior of the mass eigenstates determined by their component mixtures of the underlying particles [17].

The squarks and sleptons can mix in similar ways. Their right and left handed versions can mix to give us the mass eigenstates we label with numbers $\left(\widetilde{\tau}_{1}, \widetilde{\tau}_{2}\right)$. Depending on the specific SUSY breaking theory, the mass eigenstates are split in proportion to the standard model fermion mass, and one of the many new SUSY parameters called $\tan \beta$ :

$$
\tan \beta \equiv \frac{v_{u}}{v_{d}}
$$

Where $v_{d}=\left\langle H_{d}^{0}\right\rangle$ and $v_{u}=\left\langle H_{u}^{0}\right\rangle$ are the vacuum expectation values of the respective Higgs fields. So, depending on the SUSY breaking scheme, for large fermion 
masses and large values of $\tan \beta$, we might expect the lower end of the SUSY particle spectrum.

The model described above is the minimal supersymmetric extension of the standard model (MSSM). All told, it contains 105 new parameters, some of which are constrained by experiment, but most of which need to be measured. It must be noted that testing a theory with this many free parameters is nearly impossible, so some creative benchmark must be used.

\subsubsection{R-Parity and the LSP}

The Lagrangian for the MSSM follows the same general format and reasoning of the standard model, however it also allows for effects like the inclusion of terms that violate lepton or baryon number. The combination of these terms may lead to proton decay at tree level through the SUSY scalar partner of the down quark. This process is tightly bound by experiments measuring the lifetime of the proton, and hence would be problematic for the MSSM. The solution is to introduce a new symmetry called R-parity [18], a conserved quantum number defined as:

$$
R=(-1)^{3(B-L)+2 s},
$$

here $B$ and $L$ are the baryon and lepton numbers and $s$ is the spin of the particle. As we can see, all standard model particles will then have $R_{p}=+1$ while all SUSY particles will have $R_{p}=-1$.

The consequences of such a conserved number are vast. Firstly, when we produce SUSY particles at colliders, we produce them in pairs. All SUSY particles will then decay to a final state with at least one SUSY particle left in the decay. The lightest SUSY particle (LSP) will be stable and not decay further into standard model particles. Indeed the LSP is a prime candidate for Dark Matter. It is a massive stable particle that will only interact with standard model particles through gravity.

\subsubsection{Production of Chargino and Neutralinos}

We ultimately will search for a dilepton signal coming from the decay of $\tilde{\chi}_{1}^{ \pm} \tilde{\chi}_{2}^{0}$, so we must study how these are produced in $p \bar{p}$ collisions [19]. The production mechanism 
is common to all SUSY models with a broken symmetry leading to mass eigenstates that are mixes of the gauge and chiral super-multiplets. There are many production modes of different combinations of pairs of $\tilde{\chi}^{ \pm}$and $\tilde{\chi}^{0}$. For example, $\tilde{\chi}_{1}^{ \pm} \tilde{\chi}_{1}^{ \pm}$would be produced at comparable rates in $p \bar{p}$ collisions. Production of $\tilde{\chi}_{1}^{ \pm} \tilde{\chi}_{2}^{0}$ pairs have both s and t- channel modes, see Figure 2.5, although the two channels have slightly destructive interference, the s-channel dominates production.
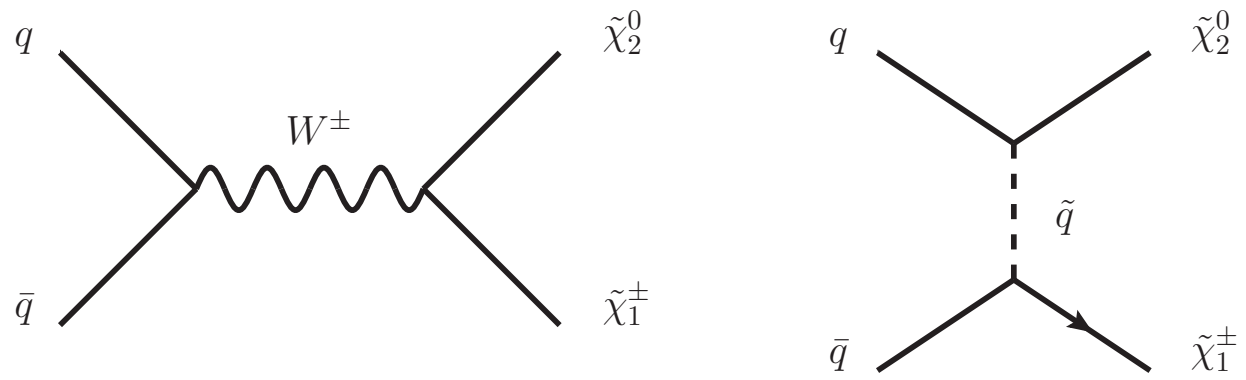

Figure 2.5: Production of Chargino $\left(\tilde{\chi}_{1}^{ \pm}\right)$and Neutralinos $\left(\widetilde{\chi}_{2}^{0}\right)$ pairs from $p \bar{p}$ collisions via the s-channel (left) and t-channel (right).

The production cross section is directly related to the mass of the pairs. A specific choice of a specific model parameters will give a mass, and therefore a production cross section.

\subsubsection{Minimal Super Gravity}

In order to study SUSY phenomenology, we must first choose the method of SUSY breaking, that is, the $\mathcal{L}_{\text {soft }}$ term in the Lagrangian and the mechanism by which it breaks. In super gravity theories, the graviton and gravitino fields play this role [14]. As implied, the graviton is the assumed force transmitter of gravity, and the spontaneous supersymmetry-breaking occurs through gravitational strength interactions in a so-called 'hidden sector' which does not directly couple to the super-multiplets of the MSSM in the 'visible sector'.

The minimal version of super gravity (mSUGRA) greatly constrain the number of free parameters in the MSSM. The theory arbitrarily sets the gluino, wino and bino mass terms equal; it also greatly simplifies other parameters in the soft SUSY Lagrangian term.

After simplification, we are left with five parameters in mSUGRA: 
1. $m_{0}$ a common scalar mass

2. $m_{1 / 2}$ a common gaugino mass

3. $A_{0}$ a common trilinear coupling value

4. $\tan \beta$ the ratio of the vevs of the two Higgs doublets, see equation 2.26

5. $\operatorname{sign} \mu$ The sign of the Higgsino mass parameter.

The LSP in mSUGRA is the lightest neutralino $\left(\widetilde{\chi}_{1}^{0}\right)$, which, because of mSUGRA mass relations and excluded parameter space, has a very high mass $(\approx 100 \mathrm{GeV})$. This leads to a large amount of energy carried away by the LSP.

The decay chain of the $\tilde{\chi}_{\mathrm{s}}$ to standard model particles we are interested in are as follows. The second lightest neutralino decays to a lepton and a slepton $\left(\widetilde{\chi}_{2}^{0} \rightarrow l, \tilde{l}\right)$; the slepton then decays to a lepton and the $\operatorname{LSP}\left(\tilde{l} \rightarrow l, \widetilde{\chi}_{1}^{0}\right)$. There are also decays through a $\mathrm{Z}$ boson if phase space allows. See Figure 2.6 for the diagrams of this decay. The Chargino decays to a slepton and a neutrino $\left(\tilde{\chi}^{ \pm} \rightarrow \tilde{l}^{ \pm}, \nu_{l}\right)$; and again the slepton decays as above. Again, decays may go through a W boson if phase space allows. The diagrams can be seen in 2.7. It is also important to note decays may occur through all fermions, and not just leptons, although we focus on leptonic decays here.

This theory is very attractive to experimentalists [14], who can now get a handle on excluding parameter space of the theory because of the reasonable number of parameters. However, this is also one reason mSUGRA is disfavored - it has been exhaustively tested and not found by experiment.

Due to its contrived nature, mSUGRA has fallen out of favor by theorists, it is now mostly used as a sensitivity comparison between experiments. It is however, useful to test gravity breaking models in general, free from the constraints of the choice of mSUGRA.

\subsubsection{Gauge Mediated Supersymmetry Breaking}

In gauge mediates supersymmetry breaking (GMSB), the $\mathcal{L}_{\text {soft }}$ term is broken not through gravity, like mSUGRA, but through gauge interactions [14]. New supermultiplets are introduced called messengers. These messenger fields couple the SUSY particles to the source of the SUSY breaking at higher scales. 


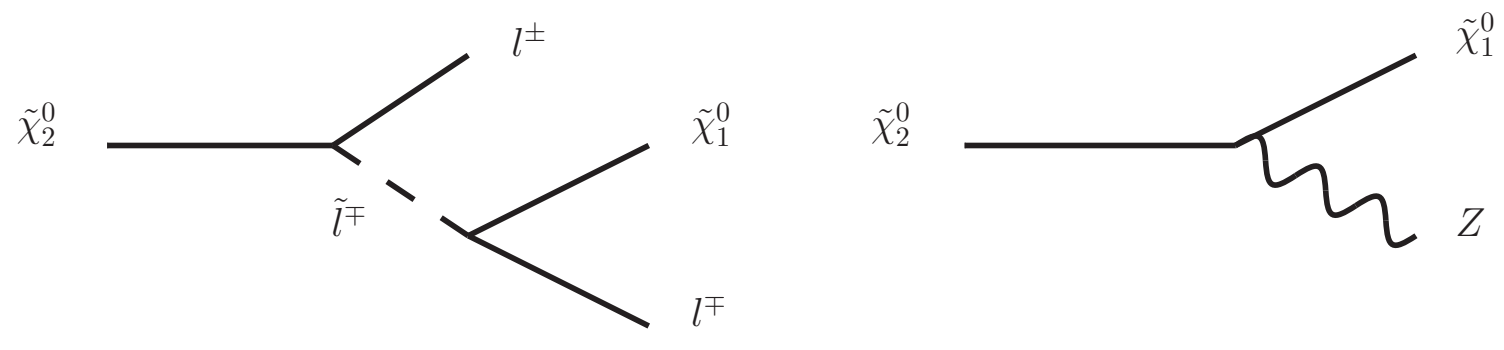

Figure 2.6: Diagrams of neutralino decay, our preferred mode (left) decays to a lepton and a slepton. Decays may go through a $\mathrm{Z}$ boson if phase space allows (left).

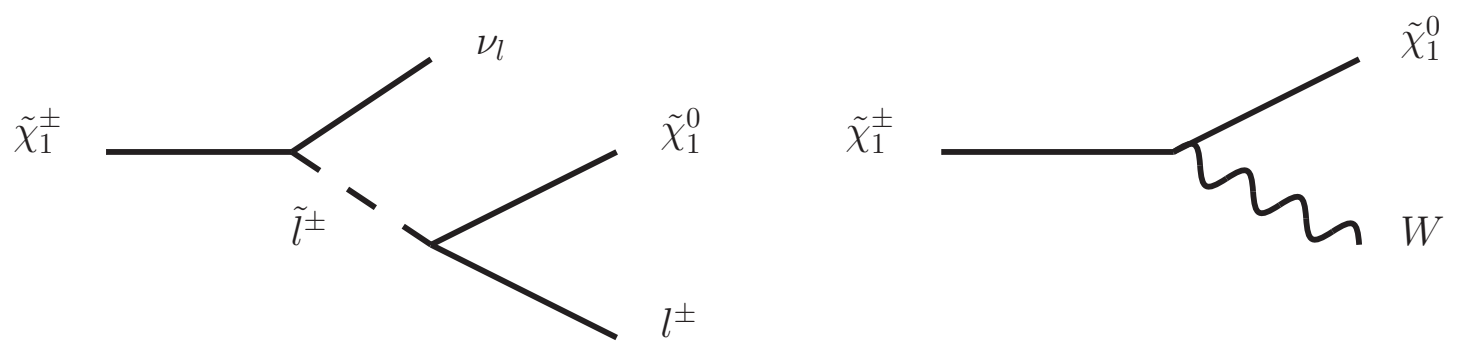

Figure 2.7: Diagrams of chargino decay, our preferred mode (left) decays to a slepton and a neutrino. Decays may go through a $\mathrm{W}$ boson if phase space allows (left).

The main effective difference between mSUGRA and GMSB is that GMSB predicts a light gravitino, having $\mathrm{keV}$ order mass. Indeed it is the LSP that escapes the detector. The next-Lightest Stable Particle (NLSP) is what classifies the types of GMSB theories decays to its super-partner and the gravitino. For our purposes detecting leptonic final states we choose the slepton as the NLSP, which will decay like $\tilde{l} \rightarrow l G$. So, the decay chain will look very similar to Figure 2.7 and 2.6, but with the final leg of SUSY branch decay going to a gravitino and not the $\widetilde{\chi}_{1}^{0}$.

\subsubsection{Simplified Models}

We have described two specific models with different SUSY breaking mechanisms in sections 2.6.6 and 2.6.5. mSUGRA in particular has only 5 free parameters, and one of those five is a sign. However, it still presents a problem to search for these theories in all possible parameter spaces. Benchmarks points have been set up, to span different interesting parameter spaces. This problem is compounded by the fact that these models have already been excluded up to high masses, and may have fallen 
out of favor. Baring a discovery, the true utility of experimental results for theorists is the ability to translate excluded mass regions into parameter space of new theories to determine if they are excluded by experiment. In mSUGRA exclusion results, for example in Figure 2.8, we have excluded a swath of parameter space for a given choice of $\tan \beta, A_{0}$ and $(\mu)$ parameters. To apply this to a new theory, one must translate the mSUGRA simplified masses to MSSM masses based on the phenomenology of mSUGRA.

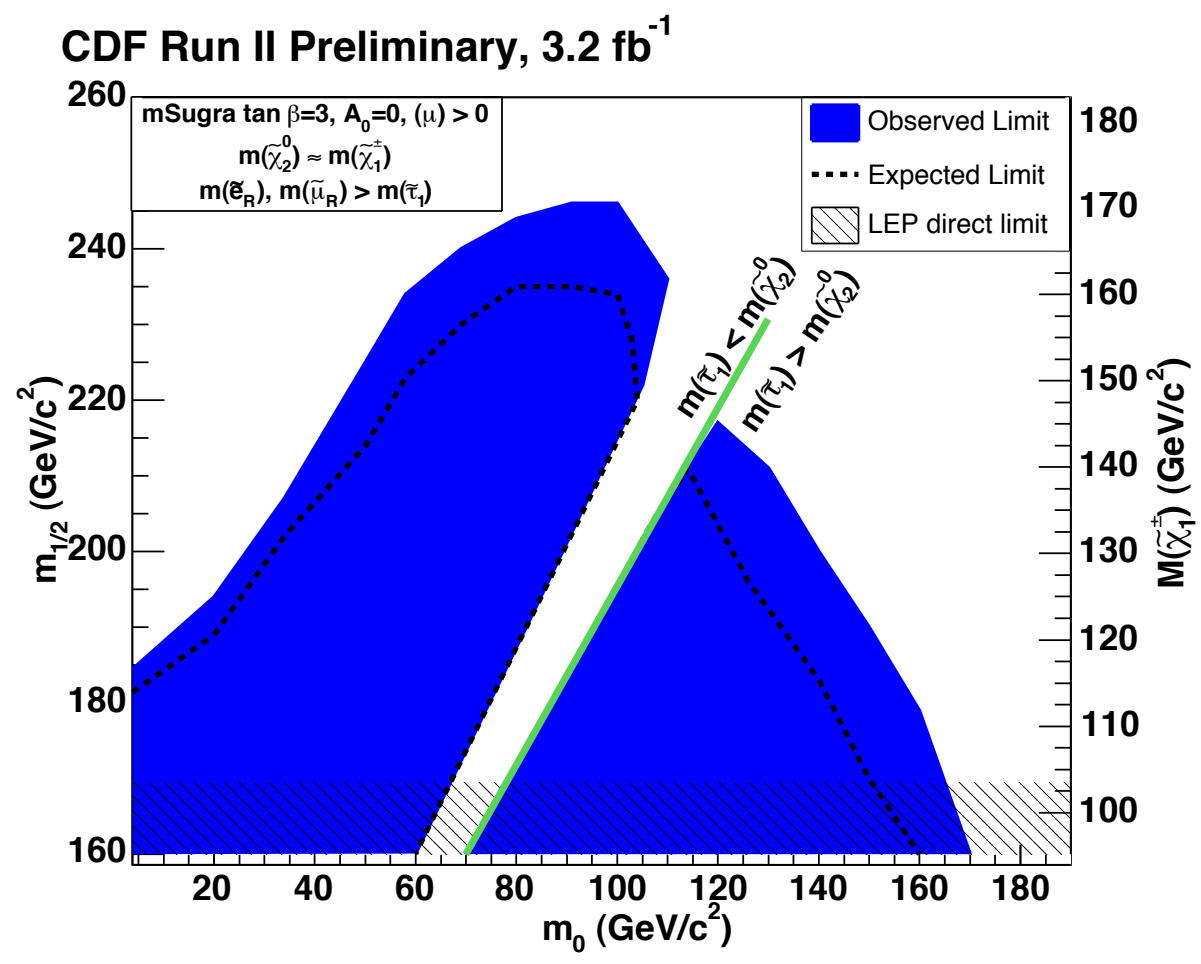

Figure 2.8: Excluded region of mSUGRA space $m_{1 / 2}$ vs. $m_{0}$ for a given choice of $\tan \beta$, $A_{0}$ and $(\mu)$ from CDF trilepton search [20].

We would like to construct simplified models of SUSY wherein we do not develop a full model of SUSY, but rather an effective theory that can be easily translated to describe kinematics of arbitrary models. We set the masses at the electroweak scale instead of describing the theory at the higher scales and we set limits as a function of these masses. We include the minimal suite of particles necessary to describe the model and effectively decouple all other particles from the process, by setting their masses $>$ TeV range. It is also possible to tune the couplings of the particles, we do this simply to mimic models that preferentially decay to taus because of a high $\tan \beta$ parameter. 
In terms of allowed decays channels of particles, specific models will determine permitted modes. Fortunately different models' SUSY breaking method will determine allowed decay modes in broad categories. Additionally, we choose a search method designed for a specific pattern of production and decay, specifically electroweak production with lepton rich decays. Therefore, we can cover a large number of model types with relatively few generic models. In this analysis we present two types of generic models inspired by the descriptions above. The first is a simplified gravity breaking model similar to mSUGRA type model from section 2.6.5. The second is a simplified gauge model, which encompasses a broad suite of theories like GMSB described in section 2.6.6.

In all these simplified models we use MadGraph/MadEvent V4.4.56 to build a model with the following generic particles: $\tilde{\chi}_{2}^{0}, \tilde{\chi}_{1}^{0}, \tilde{\chi}_{1}^{ \pm}, \tilde{l}, \nu_{\tilde{l}}, \tilde{d}, \tilde{u}, \tilde{g}$. The appropriate couplings are also created and masses of the particles specified. MadGraph/MadEvent then produces events with $\tilde{\chi}_{1}^{ \pm}, \tilde{\chi}_{2}^{0}$ pairs.

\subsubsection{Simplified Gravity Model}

The simplified gravity model is very similar to mSugra described in section 2.6.5. As is described in section 2.6.7 we decouple particles from their model specific constraints and allow their masses to run freely. Therefore we are no longer constrained by mass relations emerging from theory, such as a common relation in mSUGRA: $M\left(\tilde{\chi}_{1}^{ \pm}\right) \approx$ $2 * M\left(\tilde{\chi}_{1}^{0}\right)$. Decay channels however, are identical to mSUGRA, indeed they are generic to gravity mediated breaking models. So, for example, if $M\left(\tilde{\chi}_{1}^{ \pm}\right)+M(W)>M\left(\tilde{\chi}_{1}^{0}\right)$, the decay $\tilde{\chi}_{1}^{ \pm}+W \rightarrow \tilde{\chi}_{1}^{0}$ will occur.

The decay chain in the simplified gravity model is as follows. $\tilde{\chi}_{1}^{ \pm}$then decays to $\tilde{l}^{ \pm}, \nu_{l}$ and $\tilde{\chi}_{2}^{0}$ goes to $\tilde{l}^{ \pm} l^{\mp}$. All the sleptons decay as normal $\tilde{l}^{ \pm} \rightarrow l^{ \pm}, \tilde{\chi}_{1}^{0}$. We can tune the branching ratio to slepton flavors. For each SUSY point, we choose two branching ratios $\operatorname{BR}\left(\tilde{\chi}_{2}^{0}, \tilde{\chi}_{1}^{ \pm} \rightarrow \tilde{\tau}+X\right)=1,1 / 3$ where 1 represents a branching ratio exclusively to $\tau$ channels and $1 / 3$ represents a decay to all lepton flavors democratically. We choose the masses of the $\tilde{\chi}_{1}^{ \pm}$and $\tilde{\chi}_{2}^{0}$ to be equal. We also generally make sure $M\left(\tilde{\chi}_{1}^{ \pm}\right)+M(W)>M\left(\tilde{\chi}_{1}^{0}\right)$ to avoid intermediate decays through $\mathrm{W}$ and $\mathrm{Z}$ bosons that would severely deteriorate our acceptance due to subsequent decays to $q \bar{q}$. Decays through intermediate $\mathrm{W}, \mathrm{Z}$ and Higgs bosons are avoided.

The chosen signal points will be in a grid for final exclusion contours. A particu- 
larly interesting region is the so-called squeezed region. This is a region testing the sensitivity of phase space dilepton searches should dominate over trilepton searches. Kinematically the third lepton is very soft due to the small mass splitting between $\Delta M\left(\tilde{\chi}_{2}^{0}, \tilde{\tau}\right)$. This effect is what causes the gap region' of trilepton exclusion plots, see Figure 2.8. The dilepton search however, should have acceptance to the two higher $P_{T}$ leptons, ignoring the third.

\subsubsection{Simplified Gauge Model}

The simplified gauge model is inspired by a suite of gauge mediated SUSY breaking scenarios as described in 2.6.6. Generally, the LSP is the gravitino which is very light: in the sub-keV range [21]. Kinematically, this will be very advantageous, as less momentum will be lost through the LSP, boosting the other leptons in the event. Gauge models come in many forms, but are generally defined by their NLSP, which for our lepton rich final state is the slepton. Also, since charginos do not couple to right handed sleptons in these models, all chargino decays are to taus, so $\operatorname{BR}\left(\tilde{\chi}_{1}^{ \pm} \rightarrow\right.$ $\left.\tilde{\tau}_{1} \nu_{\tau}\right)=1$ always. The $\tilde{\chi}_{2}^{0}$ can decay to all lepton flavors. The final feature of this model is that $\tilde{\chi}_{1}^{ \pm}$or $\tilde{\chi}_{2}^{0}$ don't decay through standard model bosons, so we relax the requirement that $M\left(\tilde{\chi}_{1}^{ \pm}\right)+M(W)>M\left(\tilde{\chi}_{1}^{0}\right)$ or heavier bosons like the $\mathbf{Z}$ or Higgs.

Again, for each point we alter the couplings to adjust the branching ratio to taus, but hold $\operatorname{BR}\left(\tilde{\chi}_{1}^{ \pm} \rightarrow \tilde{\tau}_{1} \nu_{\tau}\right)=1$ as described above. We again cover a 'squeezed region' where $\Delta M\left(\tilde{\tau}, \tilde{\chi}_{2}^{0}\right)$ is small. 


\section{Chapter 3}

\section{Experimental Setup}

The large mass SUSY particles we wish to discover emerge from high energy collisions of standard model particles. Fermi National Accelerator Laboratory's Tevatron is currently one of the highest energy particle colliders in existence; the circular collider located in Batavia, Illinois has a $6.3 \mathrm{~km}$ circumference. It collides protons (p) and antiprotons $(\bar{p})$ at a center of mass energy of $\sqrt{s}=1.96 \mathrm{TeV}$. It has been in operation producing collisions since 1985 and is scheduled for termination in September of 2011.

Collisions are produced at two points along the ring of the Tevatron; two particle detectors CDF and DØ are built to encompass the two points of collisions. This thesis uses data collected by the CDF detector: a cylindrical detector consisting of a silicon tracker, a tracking chamber, solenoid magnet, calorimeters and muon systems.

This chapter describes Fermilab's chain of accelerators, including the pre-accelerators, antimatter production and storage leading up to injection and collision in the Tevatron. I then describe the CDF detector in detail including all the relevant subdetectors and how they work in concert to produce the story of a collision event. I then describe the triggering system examining how its hardware is designed to make quick decisions about which of the many events to record.

\subsection{The Accelerator Chain}

Fermilab's accelerator chain refers to the system of interconnected accelerators used in concert to produce particles suitable for collision at the experiments. Generally, 
the accelerator chain is multiple use - it provides beams of different energy and composition to several experiments at the lab. Its main purpose however is to produce high energy $p \bar{p}$ collisions, which is the process we will describe here. Particles start in an electrostatic accelerator before entering a linear accelerator that feeds a circular booster increasing the energy of the particles at each step. Further acceleration occurs in the much larger Main Injector where some protons are then used to produce antiprotons which are then stored. When a store of suitable $p$ and $\bar{p}$ particles are ready, they are injected into the Tevatron, accelerated, and forced to collide. A schematic of the accelerator chain is given in Figure 3.1.

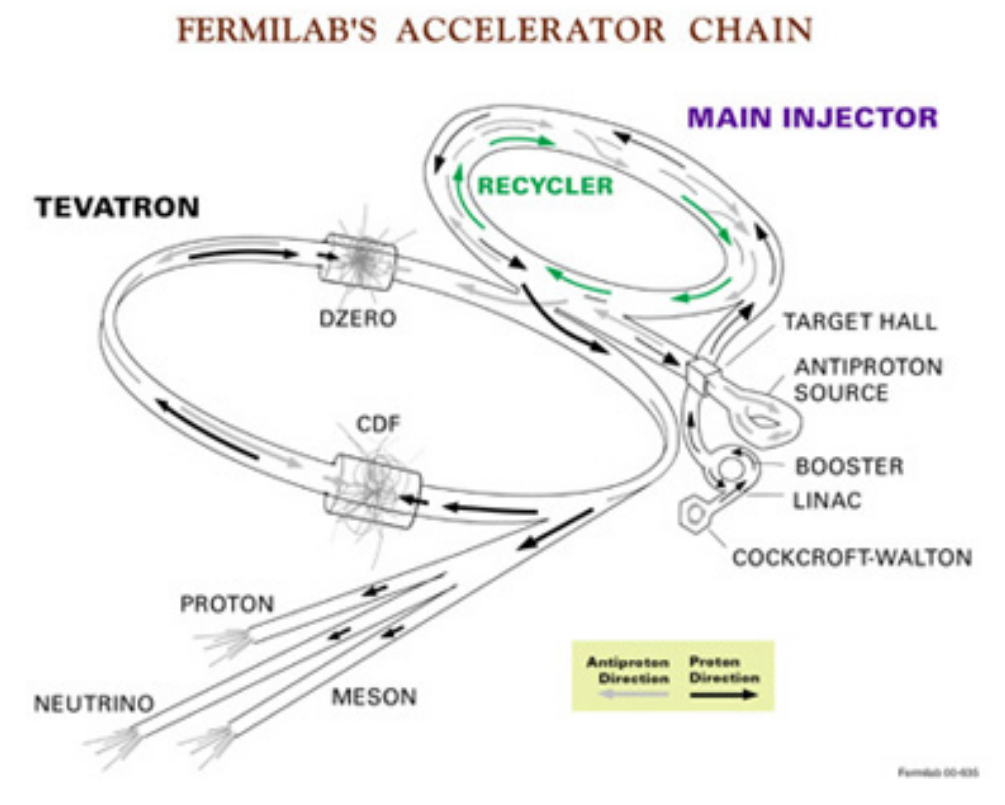

Figure 3.1: Fermilab's acceleratior chain. (Image courtesy of Fermilab Visual Media Services)

\subsubsection{Pre-Acceleration: The Linac, Booster and Main Injector}

The pre-accelerator chain consists of a series of machines that accelerate what will become protons to velocities close to $\beta \equiv \frac{v}{c} \rightarrow 1[22]$.

Particles start as hydrogen gas that is ionized to make $\mathrm{H}^{-}$. This is done in a Cockcroft-Walton accelerator, which consists of an electrically isolated dome floating at $V=-750 \mathrm{kV}$, in the center of a room whose walls are grounded. The ions are allowed to pass from the dome to the walls through a tube into the Linac. 
The Linac accelerates the ions to $400 \mathrm{MeV}$ in two sections. Radio Frequency (RF) signals at $201 \mathrm{MHz}$ are fed into accelerating cavities at $15 \mathrm{~Hz}$. Initially, the Linac uses power amplifier tubes and then uses more current Klystron technology in the latter section of the Linac. The ions are then introduced into the booster.

The next accelerating structure is the booster, a $471 \mathrm{~m}$ circumference synchrotron. Upon entering the structure, ions are sent through a foil stripping off the electrons. The remaining protons are accelerated to $8 \mathrm{GeV}$ in successive turns and sent through a transfer line to the Main Injector.

The Main Injector (MI) is a vital part of the accelerator chain which acts as a switchyard for protons. Its main function is to accelerate particles to higher energies and store both protons and antiprotons. The MI is housed in a $6 \mathrm{~km}$ circular tunnel tangential to the Tevatron into which it injects accumulated stores of protons and antiprotons. When used to introduce protons and antiprotons to the Tevatron it accelerates them to $150 \mathrm{GeV}$. It also provides protons to be used for the production of the antiprotons in which case it operates at $120 \mathrm{GeV}$ directing its proton beam to the antiproton source and then later receiving stores of antiprotons from the accumulator.

At the start of a store, or shot of particles used to load the Tevatron, the MI accepts protons from the booster, accelerates them, and injects them at $150 \mathrm{GeV}$ into the Tevatron. It then accepts antiprotons from the accumulator at $8 \mathrm{GeV}$, which circulate in the opposite sense in the MI, accelerates them, and injects them into the Tevatron. In this way it acts as the main interface between the all the pre-accelerators and the Tevatron.

\subsubsection{Antiproton Production and Storage}

Antiproton production rate is the limiting factor in the ultimate luminosity of the Tevatron. The antiproton source is a synchrotron accepting $120 \mathrm{GeV}$ protons from the MI which hit a target station made of nickel alloy. These collisions produce a spray of many types of particles; antiprotons are selected through their appropriate charge and momentum after being bent by a magnet. The result is a stream of 8 $\mathrm{GeV}$ antiprotons that are introduced into the debuncher - a synchrotron in the same tunnel as the target. The debuncher cools the antiproton bunches coming off the target, reducing their momentum spread.

The cooled antiprotons are stored in the Accumulator, housed in the same tunnel 
as the debuncher. Additional cooling takes place until the antiprotons can be sent to the recycler for longer term $(\approx 10$ hour) storage.

The Recycler is housed in the same tunnel as the MI, it uses permanent magnets to store higher intensities of $8 \mathrm{GeV}$ antiprotons than is possible in the Accumulator. As the intensity of antiprotons increases, additional cooling is needed to decrease emittance and avoid excessive antiproton losses. Electron cooling introduces a very low emittance electron beam collinear to the antiproton beam; momentum transfer between the two particle types cools the antiprotons. This is a newly implemented upgrade that has recently led to a dramatic jump in Tevatron instantaneous luminosity.

\subsubsection{The Tevatron}

The main accelerator at Fermilab, the Tevatron has a circumference of $2 \pi \mathrm{km}$. The large radius is needed due to bending dipole magnet limitations as well as synchrotron radiation losses from the co-circulating beams of protons and antiprotons. The magnets are cryogenically cooled niobium/titanium alloy superconductors kept at a temperature of $4.6 \mathrm{~K}$ by liquid helium. The ring consists of 774 dipole magnets, used to bend the beam around the tunnel, and 240 quadrupole magnets used to focus the beam. As noted above in Section 3.1.1 the Tevatron is fed $150 \mathrm{GeV}$ beams from the MI. During a ramping process, the beams are accelerated to 980 $\mathrm{GeV}$, where they remain for several hours during collisions until component failure or loss of useful luminosity. The particle structure inside the accelerator is 3 trains of 36 particle bunches separated by 396 ns with about $3.0 \times 10^{11}$ protons/bunch and $7.0 \times 10^{10}$ antiprotons/bunch. The crossing rate at each detector is about $1.7 \mathrm{MHz}$. The protons and antiprotons circulate in the same structure in opposing directions traveling in helictical paths, electrostatic separators ensure the beams do not interfere as they cross. At two points along the beam, the center of the CDF and D $\varnothing$ detectors, magnets focus and tighten the helictical structure to a point, initiating particle collisions.

\subsubsection{Luminosity and Cross Section}

Instantaneous luminosity is a quantity of the colliding beams that, when multiplied by a cross section, characterizes the rate $(\mathrm{R})$ of collisions per second $(R=\sigma L)[23]$. 
It is given by the expression:

$$
L=\frac{f_{b c} N_{b} N_{p} N_{\bar{p}}}{2 \pi\left(\sigma_{p}^{2}+\sigma_{\bar{p}}^{2}\right)} F\left(\frac{\sigma_{l}}{\beta^{*}}\right)
$$

where $f_{b c}$ is the revolution frequency $N_{b}$ is the number of bunches in either beam, $N_{p}$ and $N_{\bar{p}}$ are the number of particles in each bunch. The $\sigma_{p}$ terms are a measure of the width of the bunches and the $F\left(\frac{\sigma_{l}}{\beta^{*}}\right)$ term is a percentage based on beam focusing at the interaction point. Currently the maximum luminosity achieved is $414.0 \mu \mathrm{b} / \mathrm{s}$ See Table 3.1 for the parameters of the Tevatron in the Run II configuration.

\begin{tabular}{l|c}
\hline \hline Parameter & Run II \\
\hline Energy $(\mathrm{GeV})$ & 980 \\
Number of bunches $\left(\mathrm{N}_{b}\right)$ & 36 \\
Revolution frequency $[\mathrm{MHz}]\left(\mathrm{f}_{b c}\right)$ & 1.7 \\
Bunch rms $[\mathrm{m}] \sigma_{l}$ & 0.37 \\
Bunch spacing $[\mathrm{ns}]$ & 396 \\
Protons/bunch $\left(\mathrm{N}_{p}\right)$ & $2.7 \times 10^{11}$ \\
Antiprotons/bunch $\left(\mathrm{N}_{\bar{p}}\right)$ & $3.0 \times 10^{10}$ \\
Antiproton Production Rate $(\overline{\mathrm{p}} / \mathrm{hr})$ & $2.0 \times 10^{11}$ \\
Total antiprotons & $1.1 \times 10^{12}$ \\
$\beta^{*}[\mathrm{~cm}]$ & 35 \\
\hline \hline
\end{tabular}

Table 3.1: Tevatron parameters for Run II .

The instantaneous luminosity of the Tevatron has been upgraded through the years leading to an exponential rise in instantaneous luminosity. Integrated over time we get integrated luminosity, $\mathcal{L}=\int L d t$, see Figure 3.2. As of this writing the Tevatron has delivered $11.5 \mathrm{fb}^{-1}$ of data to the experiments, on track to deliver over $12.0 \mathrm{fb}^{-1}$ by the time the Tevatron program is terminated at 2:00 pm September 30th, 2011. 


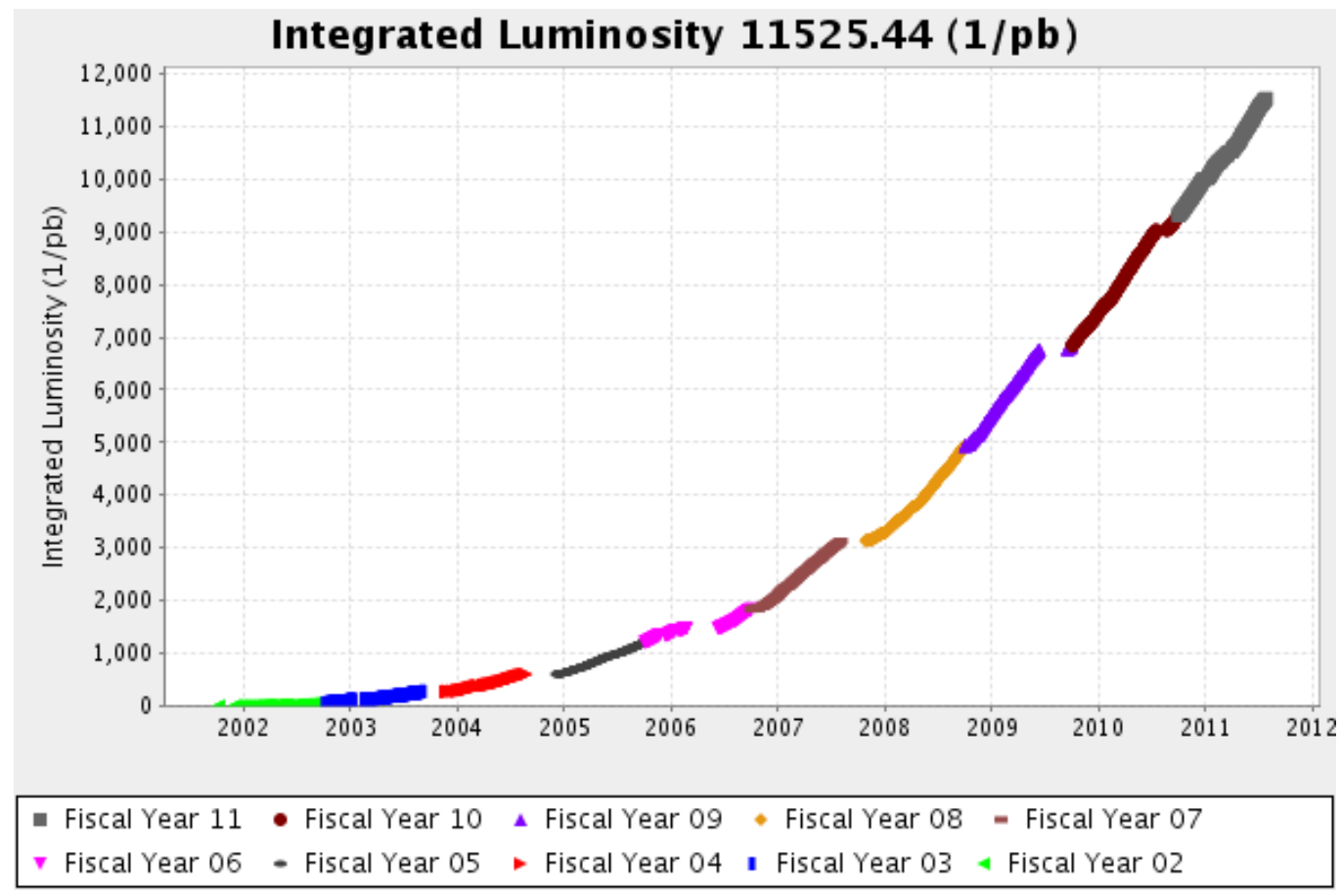

Figure 3.2: Tevatron Collider Run II Integrated Luminosity.

\subsection{The CDF II Detector}

The CDF Run II detector is a cylindrical multi-purpose particle detector designed to study $p \bar{p}$ collisions at the Tevatron [24]. It is actually a combination of many sub-detectors working in concert, with the purpose of reconstructing particles on an event-by-event basis. Although there are many sub-detectors, they can generally be categorized by their function and location relative to the beam. The inner most detector is a silicon strip detector with micron level position resolution. A large gas filled tracking chamber surrounds the silicon and provides additional charged particle tracking resolution. These two tracking systems sit emerged in a superconducting solenoid magnet that bends the tracks of the charged particles inside, allowing momentum and charge determination for tracks. Outside of these systems a calorimeter measures the energy of incident particles. The outermost layer consists of drift chambers that tag muons escaping the inner detector. A schematic of the CDF Run II detector can be seen in Figure 3.3.

CDF uses a cylindrical coordinate system wherein the positive $z$-axis lies along the direction of the incident proton beam, $\phi$ is the azimuthal angle and $\theta$ is the polar 


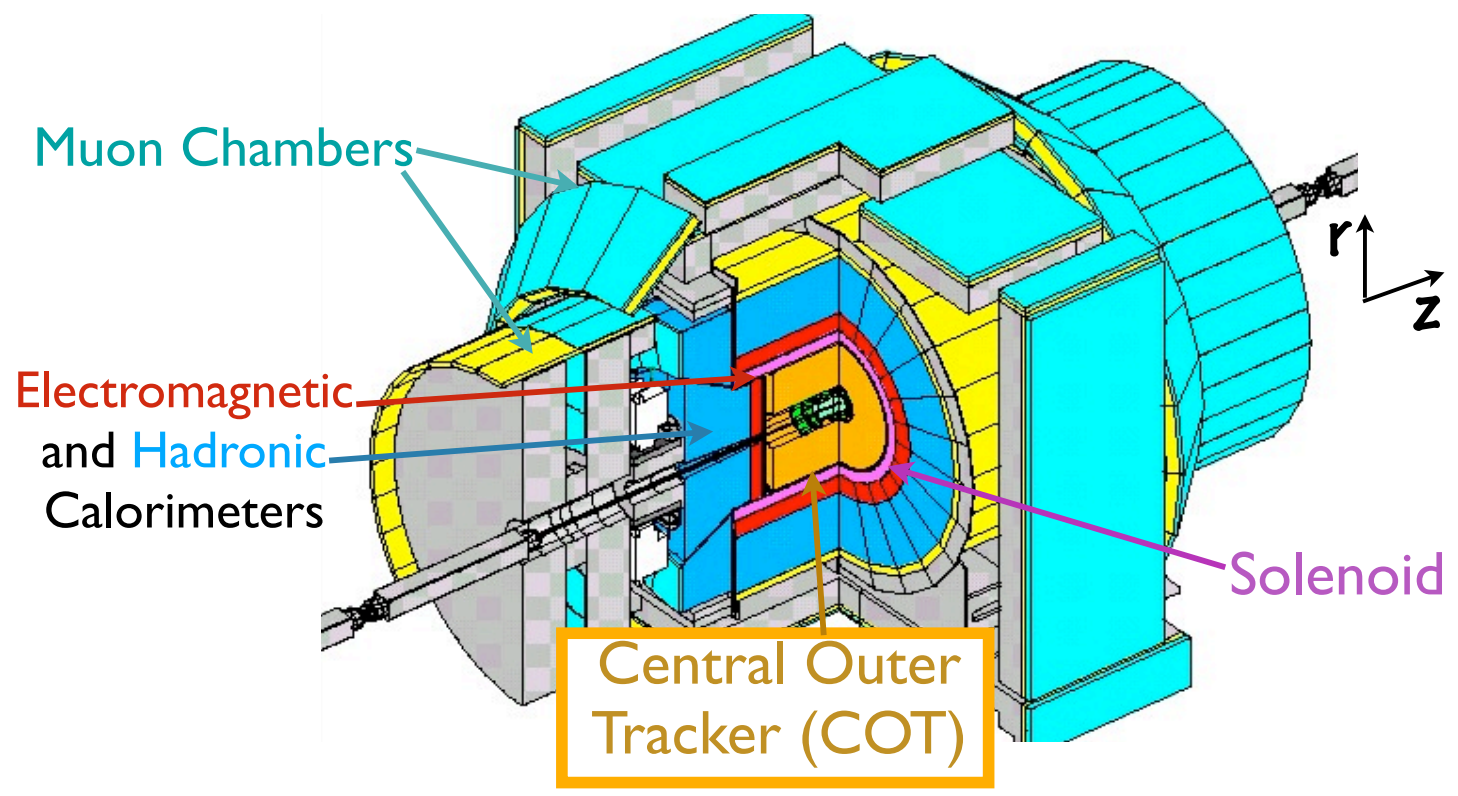

Figure 3.3: CDF Run II Detector Overview.

angle. We commonly use pseudorapidity as opposed to $\theta$ where:

$$
\eta=-\ln \left[\tan \left(\frac{\theta}{2}\right)\right]
$$

is the definition of pseudorapidity.

In what follows, I will describe each of the relevant detectors of the CDF Run II detector as well as the triggering system tasked with recording interesting events in real time.

\subsubsection{The Tracking System: Silicon and the COT}

The tracking system measures charged particle tracks with great accuracy, at its center it consists of a series of delicate silicon strip detectors, these lie inside a large gas-filled tracking chamber.

The silicon system consists of three micro strip detectors [25]. The first, called Layer 00 (L00), is placed as close to the interaction point as possible to reduce premeasurement material interactions. L00 is a single sided detector glued directly to the outside of the Beryllium beam pipe at a radius of $r=1.5 \mathrm{~cm}$. It provides a measurement in $r$ and $\phi$ of a charged track. Outside of L00 is a larger detector called 
the Silicon Vertex Detector (SVXII), it has five layers of double-sided strip sensors and spans $2.5 \mathrm{~cm}<r<10.6 \mathrm{~cm}$. There is a slight angle between the $p$ and the $n$ doped strips, enabling stereo $(z)$ resolution. The SVX II is the only detector used to trigger on events with displaced vertices. The ISL is the largest of the silicon detectors, it consists of three double-sided layers, two of which provide forward coverage (high $\eta$ ). The ISL extends to $r=29 \mathrm{~cm}$ and serves to connect the silicon tracks to the tracking chamber.

Immediately outside the ISL, the Central Outer Tracker (COT) begins [26]; it is a Argon-Ethane filled cylindrical chamber spanning $40 \mathrm{~cm}<r<137 \mathrm{~cm}$ radially, \pm $3.1 \mathrm{~m}$ in $z$ and extends to $|\eta|=1.0$. As a charged particle passes through the COT, gas is ionized and ions are directed by an electric field to sense wires.

The wires in the COT are arranged in 8 super-layers (SL) as can be seen in Figure 3.4. The odd SLs are alternately tilted at $\pm 2^{\circ}$ relative to $Z$ providing the ability to measure the $Z$ position of tracks. Each SL is segmented in $\phi$ into cells, or groupings of alternating potential and sense wires. Potential wires set up an electric field together with other structural components in the cell.

As a particle passes through the COT, the argon-ethane gas is ionized and the ions drift at $50 \mu \mathrm{m} / \mathrm{ns}$ along the electric field to the sense wires. Because the COT is immersed in a solenoid magnet, the ions drift at a Lorentz angle of $35^{\circ}$ relative to the radial, and the super-cells are tilted to accommodate. The position resolution of the COT is $140 \mu \mathrm{m}$, corresponding to a momentum resolution of $\sigma\left(P_{T}\right) / P_{T}^{2}=$ $0.15 \% / \mathrm{GeV} / c[26]$.

\subsubsection{The Calorimeters}

The calorimeter system sits just outside the solenoid and is responsible for destructively measuring the energy of incident particles such as electrons, photons, and hadronic jets [27]. As the particles collide with the material in the calorimeters, a shower of particles develop. These particles are sampled by embedded scintillators whose signals are read by PMT's.

The calorimeter system is arranged in wedges or segments in $\eta \times \phi$ space extending all $2 \pi$ of $\phi$ and $|\eta|<1.1$. A wedge in the central system is $15^{\circ}$ in $\phi$ and 0.11 units of $\eta$, The plug calorimeters extend further in $1.1<|\eta|<3.6$ and operate similarly to the central calorimeters, but are not used to explicitly reconstruct objects in this 


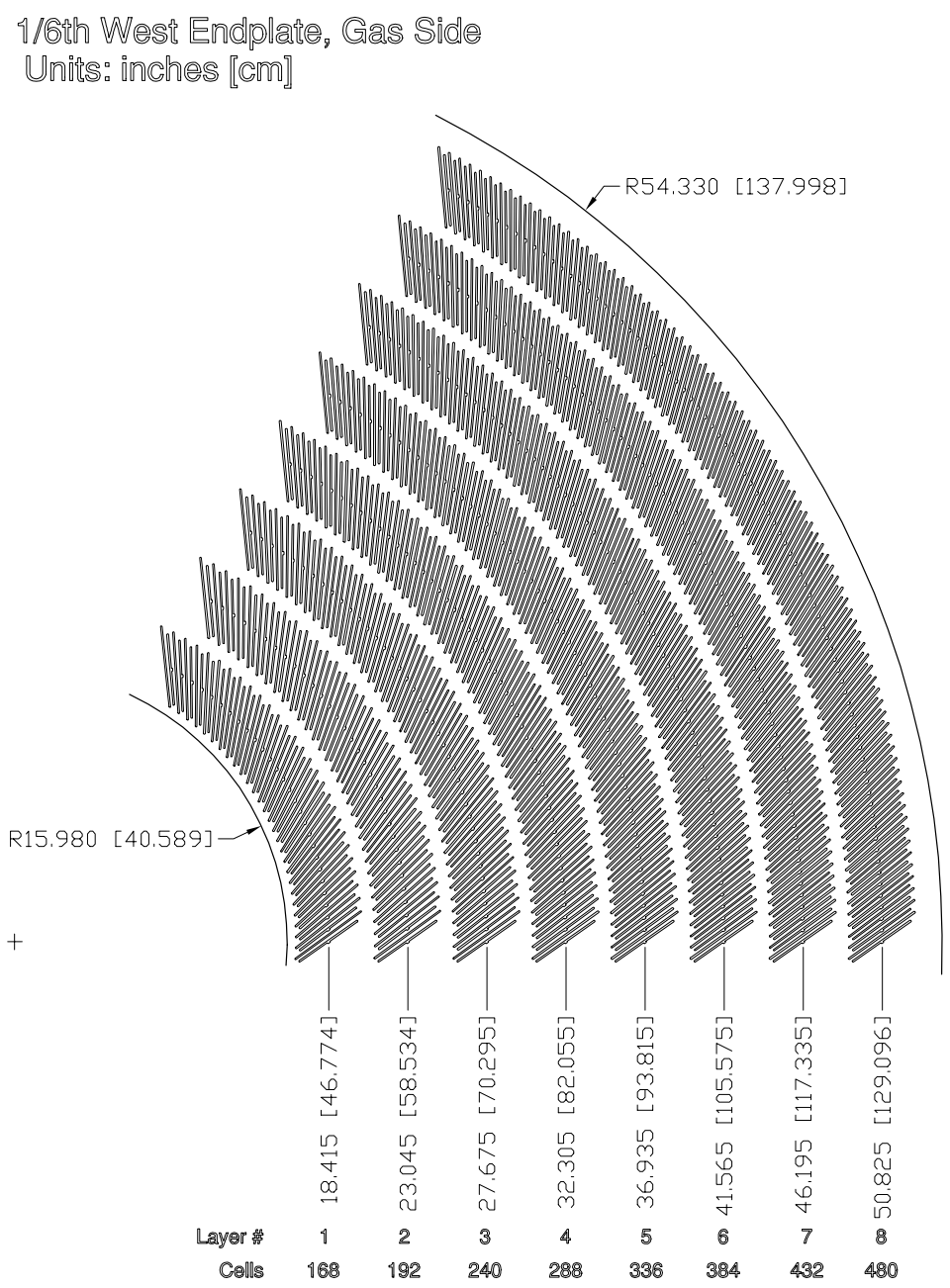

Figure 3.4: Wire planes on a COT endplate.

analysis.

Each tower has three main components: an electromagnetic calorimeter (CEM), a shower maximum (CES) detector, and a hadronic calorimeter (CHA). Electromagnetic objects such as electrons and photons are normally absorbed in this first CEM stage. The CEM has alternating layers of scintillator and lead, spanning 18 radiation lengths $\left(X_{0}\right)$. The CHA contains alternating layers of steel and scintillator and is 4.7 interaction lengths deep. Light absorbed by the scintillating layers is combined in a wedge and directed in wavelength-shifting fibers to photomultiplier tubes. Calibration test beam measurements of the calorimeters upon construction correlate sampled light to particle energy. Energy resolution for electrons and photons in the CEM is $\frac{\sigma\left(E_{\mathrm{T}}\right)}{E_{\mathrm{T}}}=\frac{13.5 \%}{\sqrt{E_{\mathrm{T}}}} \oplus 1.5 \%$. Pions were used to calibrate the CHA, whose energy resolution is $\frac{\sigma\left(E_{\mathrm{T}}\right)}{E_{\mathrm{T}}}=\frac{50 \%}{\sqrt{E_{\mathrm{T}}}} \oplus 3 \%$. 
The shower maximum detector (CES) is embedded inside the CEM at a radiation length of $5.9 X_{0}$, precisely where we expect the maximum deposition of energy from a shower of particles resulting from a $15 \mathrm{GeV}$ electron. Wires and strips arranged orthogonally to each other provide a crude 2-D reconstruction of position and extent of the shower.

\subsubsection{The Muon System}

Charged particles emerging from the calorimeter system are most likely muons. Drift chamber detectors and scintillators are positioned as the outer most layer of the CDF detector to tag muon candidates. The muon detectors used in this analysis are the Central Muon Detector (CMU) the Central Muon Upgrade Detector (CMP) and the Central Muon Extension Detector (CMX).

The CMU consists of four layers of staggered single wire drift chambers with a readout. They are embedded just on top of the central calorimeter wedges and have a coverage of $|\eta|<0.6$. The $\phi$ position can be calculated from the drift time, and $z$ position can be found through charge division between readout on respective ends of the chambers.

The CMP is arranged in rectangular geometry around the whole detector, it provides confirmation of CMU tracks as well as background rejection. This system is again a series of drift tubes four deep, resembling the CMU.

The final muon system is the CMX which provides extended coverage from $0.6<$ $|\eta|<1.0$, it is shaped in large conical arches on either side of the detector. Because of physical constraints, it is not instrumented in several $\phi$ regions. Complete muon geometrical coverage can be found in Figure 3.5.

\subsubsection{Luminosity Measurement}

The luminosity of CDF is measured by gas Cerenkov counters called the CLC [28-30]. They are designed to measure luminosity using inelastic collisions, so they are positioned at large $\eta, 3.7<|\eta|<4.7$. There are two detector assemblies placed on each side of the beam; each assembly contains 3 concentric rings of 16 counters each. Each counter is a long Mylar cone that directs light to PMT's at the end of the counters. 


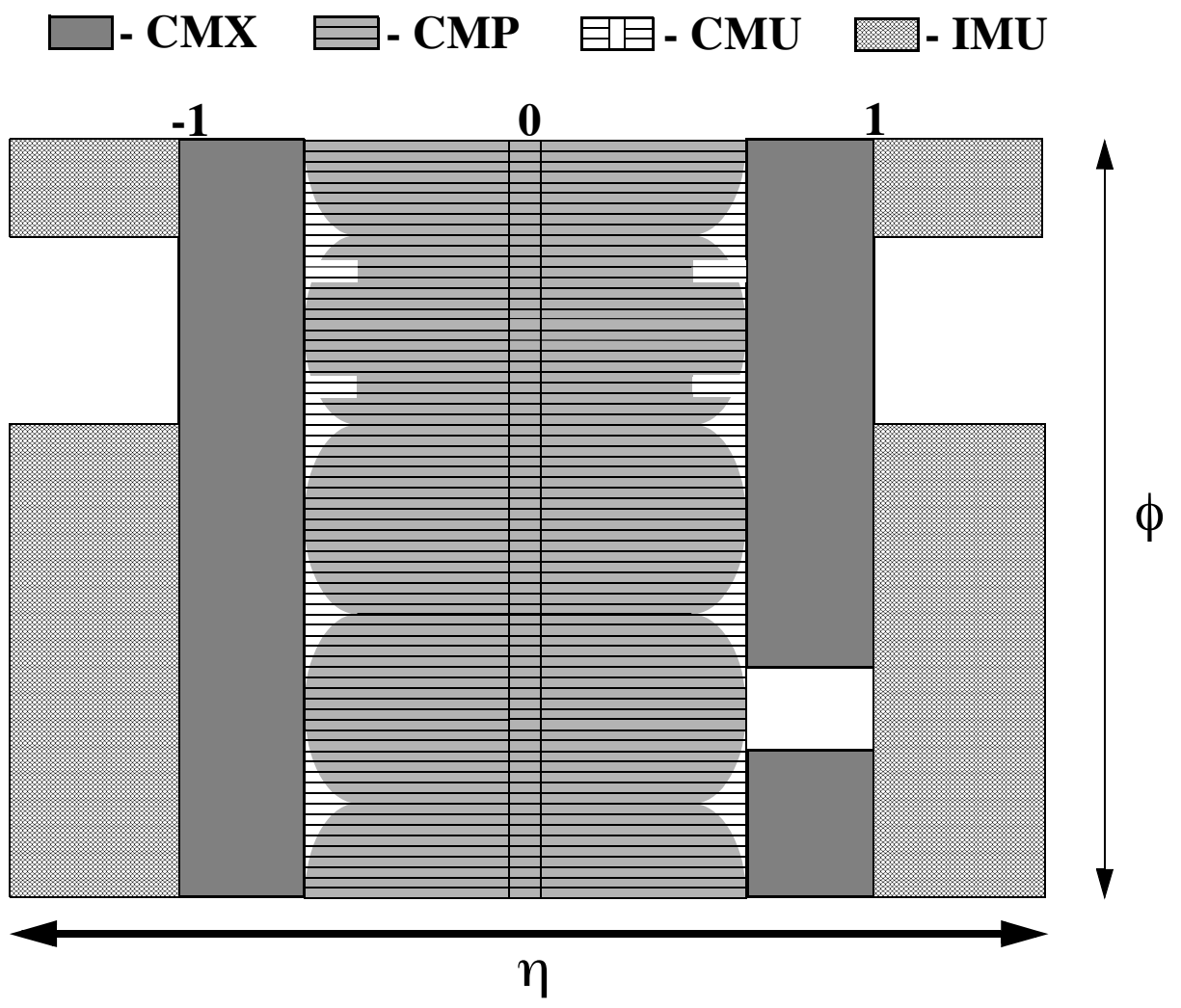

Figure 3.5: CDF Muon system coverage in $\phi$ vs $\eta$.

Luminosity is calculated with the relation:

$$
\mathcal{L}=\frac{\mu \cdot f_{b c}}{\sigma_{i n}}
$$

where $\mu$ is the average number of primary interactions, $f_{b c}$ is the $1.7 \mathrm{MHz}$ bunch crossing frequency of the Tevatron, and $\sigma_{i n}$ is the inelastic cross section. The value of $\sigma_{i n}$ was extrapolated from lower energy CDF Run I measurements, and then standardized with $\mathrm{D} \varnothing$, leading to a small offline correction factor of 1.019 .

The CLC measures empty crossings, that is, a bunch crossing with no interactions. An interaction is defined as a bunch crossing with hits in both sides of the CLC detector above a certain threshold. The probability of having no hits in either side of the CLC can be calculated and corrected for threshold and acceptance effects. The total systematic error on the luminosity is $5.8 \%$ combining an uncertainty on measurement of $4.2 \%$ and on the inelastic cross section of $4 \%$. 


\subsubsection{Triggering and DAQ}

The CDF triggering system is a vital part of the physics program. While the bunch crossing rate of $2.53 \mathrm{MHz}$ it is only feasible to record events at about $150 \mathrm{~Hz}$. The order of magnitude between these two numbers necessitates the prioritization of physics events as well as a filtering system that can trigger on the pre-defined desired event types. Each of the trigger levels must be efficient enough that the output accept rate is lower than the next level's input capacity, or dead-time results [31].

To realize this, CDF has constructed an intricate dead-timeless three-leveled triggering system, see Figure 3.6. Level one is a hardware-based system that takes crude information from detector components at the crossing rate, stores the information in a pipeline, and accepts events at $25 \mathrm{KHz}$. Level two is mostly hardware based, and buffers events four deep, accepting them at $800 \mathrm{~Hz}$. Level three is a massively parallel computing farm that writes events to tape at $300 \mathrm{~Hz}$. The whole system is synchronized to the master clock of the Tevatron and controlled by the Trigger Supervisor Interface (TS).

Below I describe the abbreviated quanta of information sent to each trigger level for accept or reject decisions, following the block diagram of Figure 3.7.

\subsubsection{Level 1}

The Level 1 trigger accepts or rejects at the rate of bunch crossings. It contains a 42 clock cycle FIFO pipeline allowing it to provide trigger decisions with about $5.5 \mu \mathrm{s}$ latency at a rate typically around $25 \mathrm{KHz}$, and in this time, the input signals must be digitalized, pushed along the pipeline, have trigger primitive objects formed and sent to the counting room to form trigger objects. The trigger objects are checked against a trigger table, and a decision is sent to the TS.

The Global Level 1 decision is based on three L1 trigger systems: a calorimeter object (L1 CAL), a Muon object (L1 MUON) and a track object (L1 TRACK).

The L1 TRACK system is special in that it must be used by the other L1 systems for pointing. It detects tracks in the COT by assembling hits on the Axial and Stereo layers into tracks. The eXtremely Fast Tracker (XFT) finds tracks over a certain $p_{\mathrm{T}}$ threshold according to a lookup table of pre-determined patterns or 'roads'. The list of tracks is then sent to the XTRP which extrapolates the tracks and distributes 


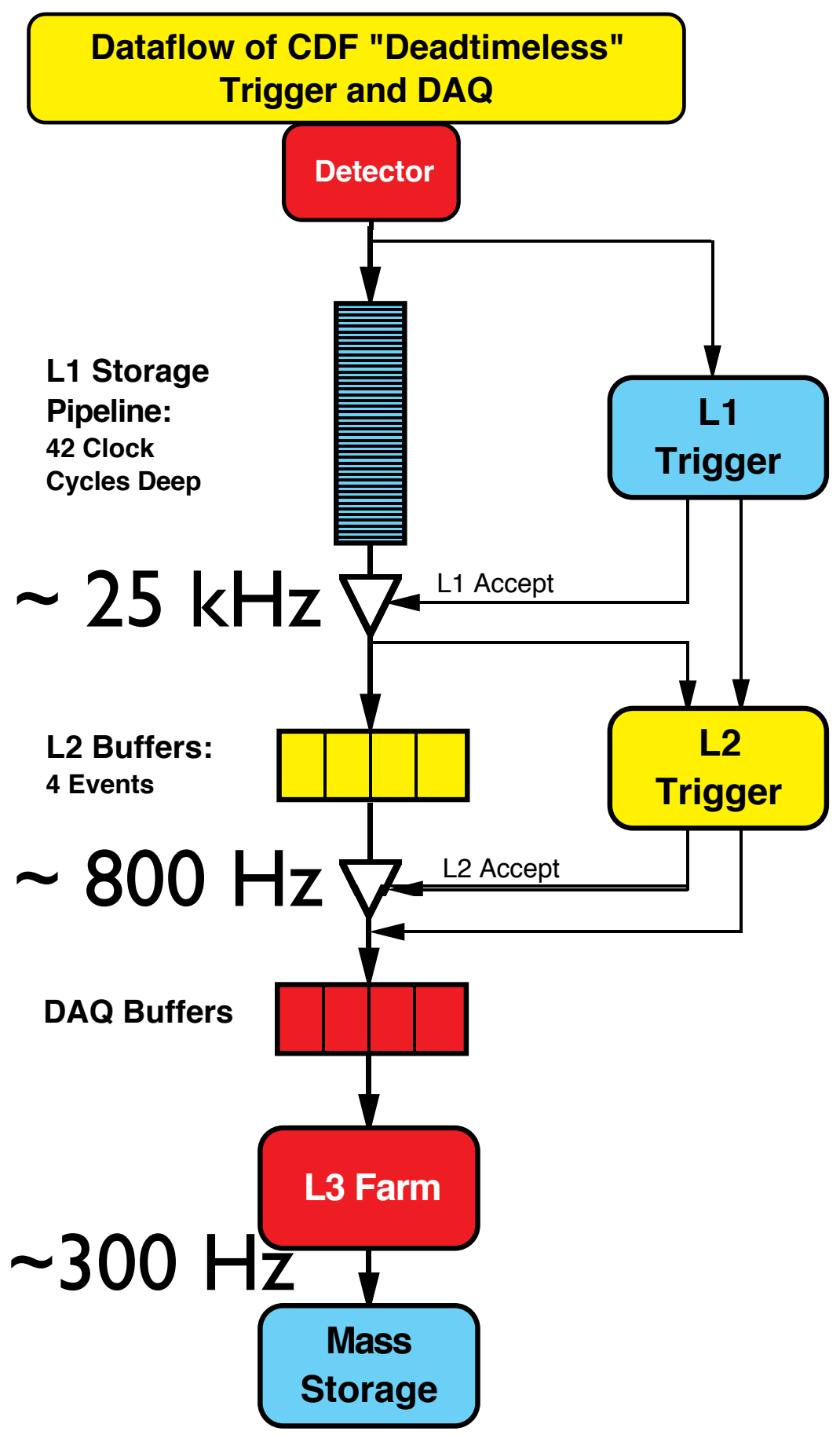

Figure 3.6: The Schematic of the three levels of the CDF dead-timeless trigger.

derivative information to all other L1 systems as well as the SVT for Level 2 decisions.

The L1 CAL calorimeter system is designed to detect both objects (photons, electrons, jets) and event level quantities $\left(\mathscr{E}_{T}\right)$. For speed, calorimeter towers are grouped into objects larger than their physical size - the trigger uses a $24 \times 24$ grid 


\section{RUN II TRIGGER SYSTEM}

Detector Elements

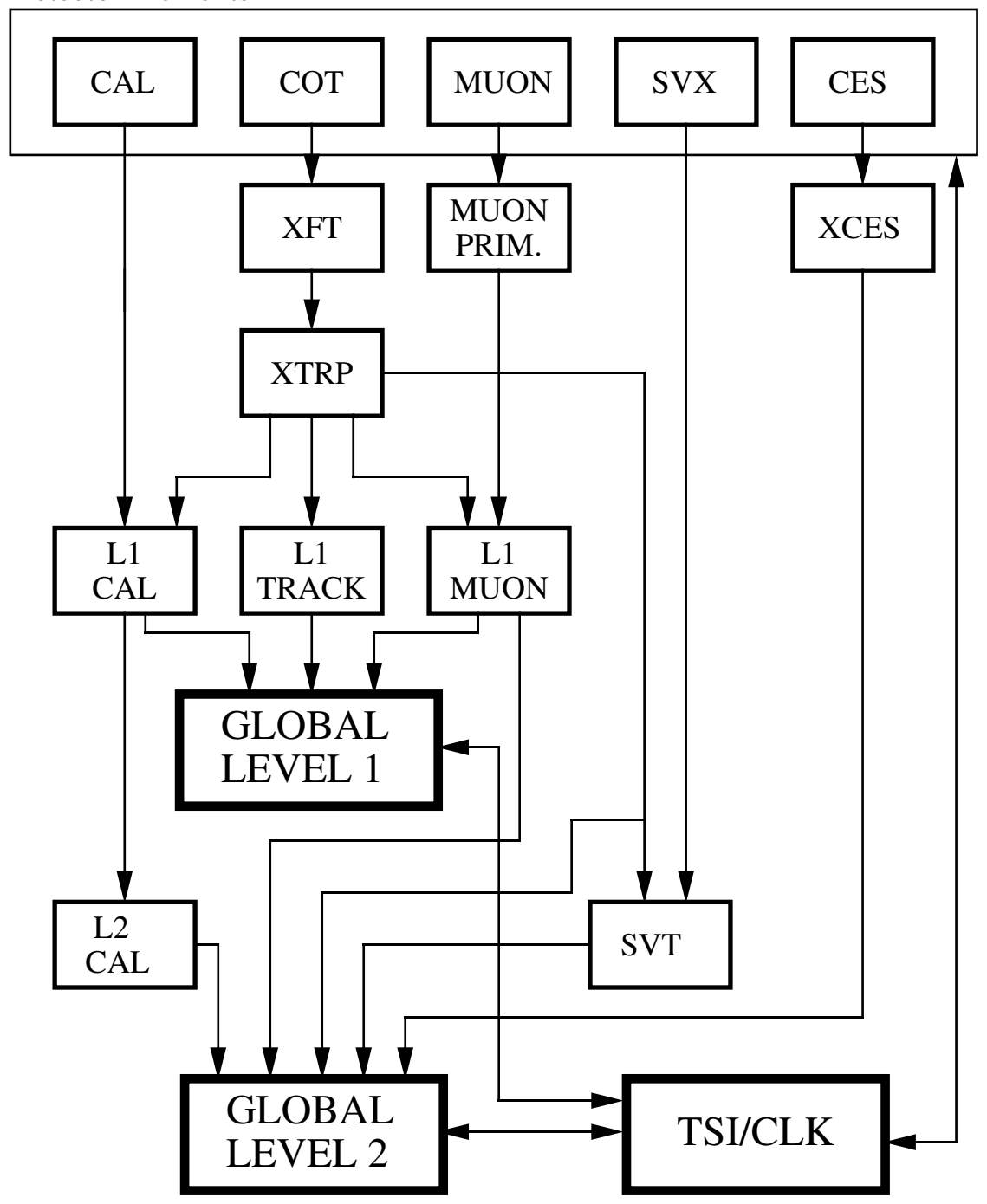

PJW 9/23/96

Figure 3.7: Block Diagram of the CDF L1 and L2 trigger system. L3 follows after an L2 Accept from Global Level 2.

of detectors in the $\phi \times \eta$ space.

The L1 MUON system uses muon primitives from all the muon systems, as well as tracks from XTRP to confirm or reject muon hit candidates. There are also scintillators embedded near the muon systems that are used to confirm or deny hits. 
These objects are then sent to Global Level 1, which checks against a trigger table and accepts or rejects the event, sending the decision to the TS. See Figure 3.7 for the schematic.

\subsubsection{Level 2}

The Level 2 trigger is an asynchronous system with a 4 event buffer the reduces the event rate to about $300 \mathrm{~Hz}$. The TS keeps track of empty buffers and sends an L1 accept command to fill the buffer with event information. If no buffer is free, CDF is dead. The whole process takes about $40 \mu \mathrm{s}$. The Level 2 system uses all the information L1 uses with additional information from the Silicon (SVX), the CES (XCES) and the Calorimeters (L2 CAL).

The L2 CAL enhances the information found at L1 by forming clusters of calorimeter towers corresponding to a characteristic particle energy deposit. Although cluster finding algorithms vary, generally there is a 'seed' tower with a certain required energy and neighboring 'shoulder' towers that combine to make a cluster.

The XCES system uses information from the CES and can provide much better position resolution than the calorimeter towers. It combines energy clusters of a certain threshold from adjacent CES wires, associates them with a track and generates the deliverable for GLOBAL L2.

The Silicon Vertex Trigger (SVT) is a vital component of the triggering system and comes into play at L2. It uses tracks from the XTRP as well as from SVXII. The SVXII system assembles silicon hits from at least four silicon layers and one XTRP track. If the pattern of hits and XTRP tracks matches a predetermined, characteristic 'road', the actual hits are retrieved from silicon buffers and calculated more precisely. The impact parameter resolution is $35 \mu \mathrm{m}$, enabling triggering on displaced vertices at L2.

If all conditions are met for a certain trigger in the L2 Trigger Table, the Global L2 issues an L2 accept to the TS. This happens at about $300 \mathrm{~Hz}$.

\subsubsection{Level 3}

Upon L2 Accept, full event data are read out from every sub-detector to be sent to the L3 farm. Like L1, if the L2 Accept rate is too large, CDF experiences dead-time. 
The Event Builder (EB) is in charge of assembling event information from every component, as well as metadata from triggering. It then sends the events to the L3 farm, consisting of a few hundred computing nodes. Each node has several seconds then to do full event reconstruction and can therefore take advantage of full detector information. If an event matches a L3 trigger Table requirement, it is sent to a data logger to eventually be written to tape for offline analysis.

The trigger table consists of many L3 trigger paths which in turn, consist of a combination of primitives from L1 and L2. One event may satisfy many trigger paths.

At high instantaneous luminosities the effective cross section, or accept rate, of the triggers increases because of the combinatorics of noise, so the trigger must be continuously tuned to avoid excessive L1 and L2 accepts. This necessitates prescaling, or auto-rejecting certain common trigger elements to avoid high dead-time. High luminosity also requires adjusting the trigger table to reflect the priority of the physics program. 


\section{Chapter 4}

\section{From Data to Physics}

After data are recorded to tape, we reconstruct and identify the physics events that took place in the collisions. The interpretation of physics from raw data to useful information is a complicated multistep process. As stated in Chapter 1, we are interested in reconstructing dilepton events with one electron or muon and at least one hadronic tau decay.

We start with the data gathered on the specific trigger path that will give us our final desired signature. The so-called 'lepton+track' series of triggers at CDF is such a trigger. It is a dilepton trigger with two legs, one leg requiring and electron or muon, the 'lepton', and the other leg requiring a track object that will be the genesis for the tau. We describe the L1, L2 and L3 requirements for this trigger, then the process of calculating the efficiency of the trigger to fire on real lepton + tau events.

After we have triggered objects, we need to reconstruct and identify real physics objects: electrons, muons, taus, and hadronic jets. This necessitates constructing a long series of cuts in each detector that distills each object based on the detector response to their intrinsic properties. We also measure the efficiency and misidentification rate of our detector to real objects. Finally we construct event level objects such as vertices and $\mathbb{E}_{T}$. 


\subsection{Data and Monte Carlo Used}

The data used was taken by the CDF detector from 4 Feb, 2002 until 25 Feb, 2010 representing $\mathcal{L}=6760 \mathrm{pb}^{-1}$ of raw data. We then filter out the runs of data where the used detectors were not validated as fully operational; to do this we apply a good run list (version 33). This list includes all electron/muon detectors and ignores the requirement for silicon functionality. The list of runs and segments is used to calculate the luminosity when associated with a certain trigger path.

We simulate common standard model processes using Monte Carlo events to later use in understanding the complete composition of our data. We generate events through a multi-step process. Firstly, we use PYTHIA [32] to generate random $p \bar{p}$ collisions of certain standard model processes. PYTHIA uses leading order matrix calculations for hard scattering events and parton showering for radiation effects. 'Truth' information refers to the actual generated object before detector simulation. For signal events, we generate our own models with MADGRAPH/MADEVENT [33], the events are decayed by BRIDGE [34] and then fed to detector simulation like the background events. The software package GEANT3 [35] is used to simulate the interaction of the particles with the complete detector simulation, this give us detector level hit information. We then process these events with the exact same methods and algorithms we use to process real data. We also take into account run dependent beam conditions, like luminosity profile and beam offset. All the samples were generated using a consistent parton distribution function, CTEQ5L [36].

\subsection{Trigger Paths}

This analysis used the 'lepton + track' triggers where the lepton can be a CEM, CMX or CMUP lepton, and the track will become a tau lepton in the analysis. The abbreviated name of the lepton is associated with the detector in which it was detected. Electrons that lose all energy in the electromagnetic calorimeter are Central Electromagnetic objects (CEM); muons are either detected in the overlapping CMU/CMP detectors (CMUP) or in the CMX. We measure the efficiency of these triggers relative to the specific tau we will ultimately use in the analysis. As described below, there are several trigger effects that need to be taken into account.

These triggers have undergone many changes over the years to increase their purity 
and reduce their effective cross section in the trigger system. At some instantaneous luminosity a certain trigger may have an unacceptably high cross section in the trigger system, so events must be thrown out. When we throw out a certain fraction of events, it is called a prescale. When the prescale fraction adjusts during a Tevatron store relative to the instantaneous luminosity, it is called a dynamic prescale. When a trigger path is turned on after the instantaneous luminosity falls below a predetermined threshold, it is called a luminosity enabled trigger. All these effects must be taken into account when determining the luminosity of a certain trigger since we have all three of these effects in out lepton + track triggers. This is done through scripts that determine the trigger prescale at the smallest quanta it can change, called a run-section. The instantaneous luminosity is multiplied by the prescale on a per runsection basis and then integrated over all run sections to determine total luminosity.

The trigger path history is very complex for each of the types of triggers, but they can be broken down to more manageable version ranges called Categories containing substantive differences in trigger efficiency. They are listed below in Table 4.1 grouped by similar tau side trigger version types. The triggers are simply named by their most basic composition so, for example TAU_CMUP8_TRACK5_ISO_DPS corresponds to a $p_{\mathrm{T}}=8 \mathrm{GeV} / c$ CMUP muon and a $p_{\mathrm{T}}=5 \mathrm{GeV} / c$ track isolated from other tracks and the DPS signifies that it is dynamically prescaled. We avoid simultaneous overlapping triggers in any one run. Since we use common trigger efficiencies for the lepton side of the trigger, we denote trigger changes by substantive changes to the tau side.

When calculated and multiplied by a correction factor of 1.019 as described in Section 3.2.4, we have an integrated luminosity of $\mathcal{L}=6031 \mathrm{pb}$ for Electron plus track; $\mathcal{L}=5709 \mathrm{pb}$ for the CMX plus track; and $\mathcal{L}=6029$ pb for the CMUP plus track. The CMX trigger was more heavily prescaled due to its intrinsically high rates. These are the luminosities for the analysis. The electron luminosity is the quoted number for the analysis, a correction factor is applied to muon Monte Carlo to account for the prescale.

Several changes to the triggers have taken place over the years, and we will describe them here. Generally, the trigger requires a track at Level 2 of a certain quality and at Level 3 imposes an isolation cone around the track. Additional trigger object requirements are added to reduce the rate. 


\begin{tabular}{|c|c|c|}
\hline Trigger Path Family & Trigger Paths involved & Category \\
\hline \multirow[t]{3}{*}{ Electron plus track } & TAU_ELECTRON8_TRACK5_ISO v 4 to 7 & $\mathrm{I}$ \\
\hline & TAU_ELECTRON8_TRACK5_ISO v 8 to 10 & II \\
\hline & TAU_ELECTRON8_TRACK5_ISO v 12 to 14 & III \\
\hline \multirow[t]{4}{*}{ CMUP plus track } & TAU_CMUP8_TRACK5_ISO, v 2 to 7 & $\mathrm{I}$ \\
\hline & TAU_CMUP8_TRACK5_ISO, v 8 & II \\
\hline & TAU_CMUP8_TRACK5_ISO, v 9 & III \\
\hline & TAU_CMUP8_TRACK5_ISO, v 10 to 12 & IV \\
\hline \multirow[t]{6}{*}{ CMX plus track } & TAU_CMX8_TRACK5_ISO, v1 to 6 & $\mathrm{I}$ \\
\hline & TAU_CMX8_TRACK5_ISO, v7 & II \\
\hline & TAU_CMX8_TRACK5_ISO, v8 & III \\
\hline & TAU_CMX8_TRACK5_ISO_LUMI_200 & III \\
\hline & TAU_CMX8_TRACK5_ISO_LUMI_250 & III \\
\hline & TAU_CMX8_TRACK5_ISO_DPS & IV \\
\hline
\end{tabular}

Table 4.1: "Lepton plus track" trigger paths used in this analysis, grouped by common trigger features or categories.

\subsubsection{Level 2 Trigger Requirements}

A XFT track with $P_{t}>5 \mathrm{GeV} / c$ is required for all CEM triggers (category $\geq \mathrm{I}$ ) and later, categories of CMUP and CMX triggers (category $\geq$ II). There was no track requirement for muons at level 2 for category I triggers.

Starting with category II CEM triggers, the XFT track must match a cluster in the calorimeter that satisfies the requirements in Table 4.2. With the trigger system upgrade completed in 2009, more advanced clustering was available to the trigger system and the cluster requirement was changed (III).

As for the muon triggers, the trigger was upgraded to enable stereo confirmation of the track corresponding to category IV triggers above. This 3DMatch ability was added afterwards, it enables the triggering system at L2 to point to different sides of the detector, its addition increases signal purity, but has no effect on efficiency. The electron channel needed no upgrade. 


\begin{tabular}{|c|c|}
\hline \hline L2 cluster requirements (II) & L2 cluster requirements $(\geq \mathrm{III})$ \\
\hline$E_{T}>4 \mathrm{GeV}$ & $E_{T}>5 \mathrm{GeV}$ \\
Number of towers $\leq 5$ & No requirement on NTowers \\
\hline
\end{tabular}

Table 4.2: L2 cluster cuts.

\begin{tabular}{c|c|c}
\hline & Category I & Categories $\geq \mathrm{II}$ \\
\hline Track $P_{t}$ & $>5 \mathrm{GeV} / c$ & $>5 \mathrm{GeV} / c$ \\
Track $|\eta|$ & $<1.5$ & $<1.5$ \\
L3 isolation annulus & $0.175<\Delta R<0.524$ & $10^{\circ}<$ Angle $<30^{\circ}$ \\
isolation track cuts & $|\Delta Z|<15 \mathrm{~cm}$ & $|\Delta Z|<5 \mathrm{~cm}$ \\
& $p_{T}>1.5 \mathrm{GeV}$ & $p_{T}>1.5 \mathrm{GeV}$ \\
\hline \hline
\end{tabular}

Table 4.3: L3 Isolation annulus definition, and cuts for isolation tracks

\subsubsection{Level 3 Trigger Requirements}

The first change to the triggers was in the isolation cone requirement. We require that no tracks be within a $|\Delta Z|$ of a certain distance as well as a certain isolation cone. About halfway through data taking the isolation cone definition was changed and the $|\Delta Z|$ was tightened. See Table 4.3 for the definition.

\subsubsection{Trigger Efficiency Measurement}

We measure the effect of every requirement of the lepton + track triggers that might lead to inefficiency. Here we refer to tight tau objects, which we will define explicitly later in this chapter. Hadronic jet data samples are used for the measurement since they have no track requirements. The jet sample however, is dominated by fake taus introducing a bias due to track multiplicity differences in the isolation study of Level 3. The electron side cluster requirement would also be biased if measured in the jet samples.

For most studies, we select tight taus in good runs from the jet sample, which includes implementing Level 3 isolation replication. We then use this sample to measure quantities against the trigger requirements in the lepton + track triggers. 
Additional studies must be done to take into account other effects. To get the absolute normalization of level 3 efficiencies we select non-isolated $Z \rightarrow e e, Z \rightarrow \mu \mu$ events by selecting standard leptons and making a cut on the $Z$ mass window. We then make tau-like isolation cones around these leptons and measure the effect of isolation by checking if they would pass the isolation requirements in the trigger.

\subsubsection{Level 2 Efficiency Measurements}

The three requirements on level 2 that are applied to different versions of the trigger are the requirement of the existence of an XFT track; the requirement that track be SLAM confirmed; and the cluster matching requirement.

The primary cause of track finding inefficiency in the XFT is due to spacers located in the $Z=0$ plane at the location of the superlayers. It has been noticed that when a track crosses this plane at the radius of these superlayers, efficiency degrades significantly. We parameterize tracks by the radius at which they cross the plane $(R Z 0)$. Tracks that do not cross the central plane of the COT have increasing efficiency with longer distances traveled in the COT. We therefore parameterize the track finding efficiency by a constant plateau with gaussian dips at the radii of the superlayers. For normal axial tracks, the stereo layers are not used in XFT track finding, so we get four dips $(\mathrm{N}=4)$. Requiring SLAM confirmation uses the additional stereo layers, and we need 7 dips $(\mathrm{N}=7)$ for total parameterization:

$$
\epsilon(x)=\epsilon_{\text {plateau }}-\sum_{i=1}^{N} A_{i} e^{-\frac{\left(\frac{x-C_{i}}{S_{i}}\right)^{2}}{2}} .
$$

Where $A_{i}, C_{i}, S_{i}$ are constants to be fit, see Table 4.4 for these fits to Figure 4.1. Note that even though the difference between category III and II triggers happens because of a level 3 trigger change, we remeasure the L2 effects for this body of data. They are essentially unchanged.

If a track does not cross $\mathrm{Z}=0$, we parameterize by the length in $r$ and $z$ of the track in the COT, Lrz. The efficiency is fit to a turn-on curve as follows:

$$
\epsilon(x)=\frac{\epsilon_{\text {plateau }}}{1+e^{\frac{C-x}{S}}}
$$


As an additional complication, the XFT system had the option to use 3 superlayer tracks before August 2006, and only used 4 layer tracks afterwards, decreasing the efficiency. We parameterize this 4 Layer track period as follows:

$$
\epsilon(x)=\epsilon_{\text {plateau }}-A e^{-\frac{\left(\frac{x-C}{S}\right)^{2}}{2}}
$$

The path length dependence largely goes away after the removal of 3 layer tracks, see Figure 4.3 and Table 4.5. For the period that requires stereo information (category IV triggers) the efficiency is $3 \%$ lower and not shown here.
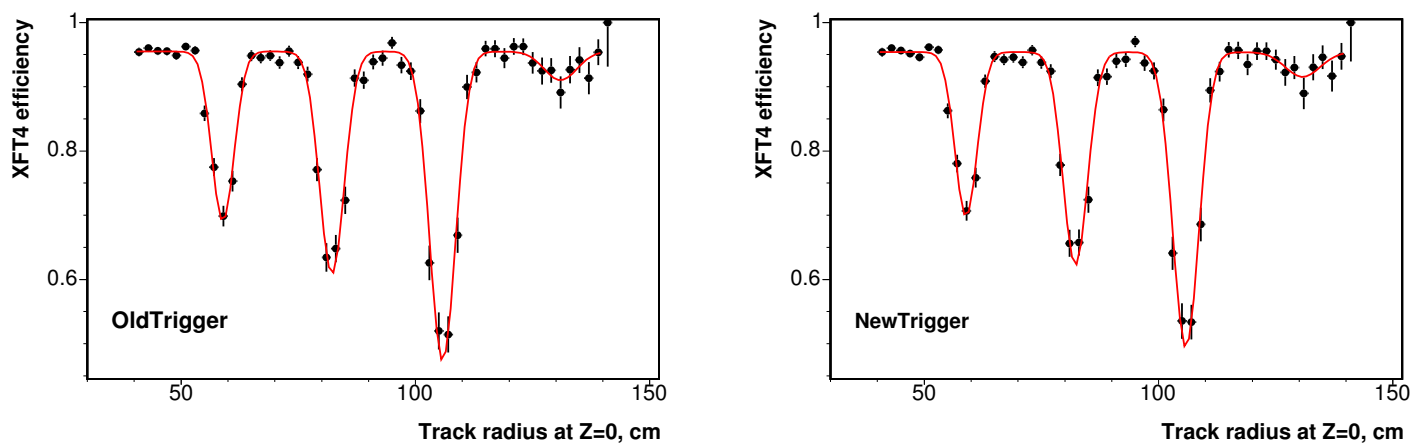

Figure 4.1: XFT track finding efficiency for tracks that cross the $Z=0$ plane for two periods corresponding to category I and II triggers. We parameterized this efficiency as a function of the radius at which the tracks cross that plane, $R Z 0$.

As for the clustering requirement, we have not found a way to measure the efficiency of the L2 cluster matching with data. Instead, we measure the L2 clustering efficiency from Monte Carlo and try to take the behavior of data versus MC into account. We derive a L2 cluster scale factor correction between data and MC that we ultimately apply to the efficiency measured from the truth information in $Z \rightarrow \tau \tau$ Monte Carlo. To derive this scale factor, we look at the cluster matching efficiency in Jets with $E_{\mathrm{T}}>20 \mathrm{GeV}$ data as well as with QCD MC. The same method is used as above, wherein we select tight taus and look at the efficiency of having a triggered L2 cluster. We then derive a Data/MC scale factor parameterized by the number of towers in the tau cluster. This scale factor corrects for differences between data and $\mathrm{MC}$ efficiencies. We then apply this scale factor to the efficiencies found for cluster 


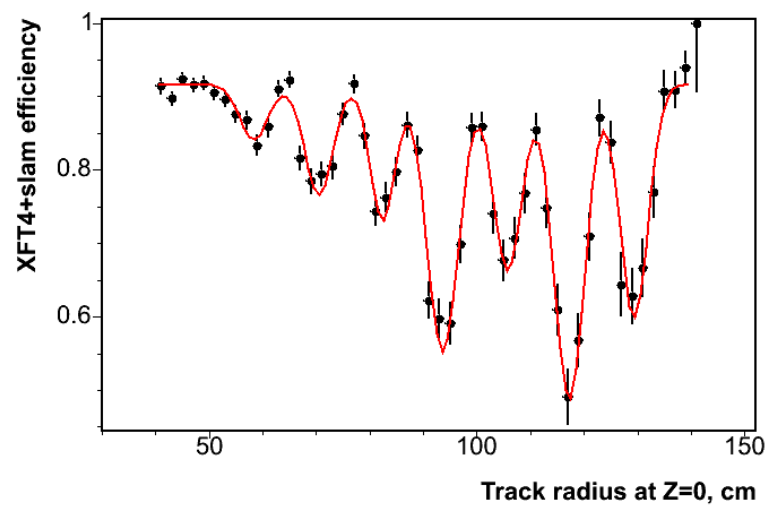

Figure 4.2: XFT track finding efficiency for tracks that cross the $Z=0$ plane wit SLAM confirmation (category IV triggers). We parameterized this efficiency as a function of the radius at which the tracks cross that plane, $R Z 0$.
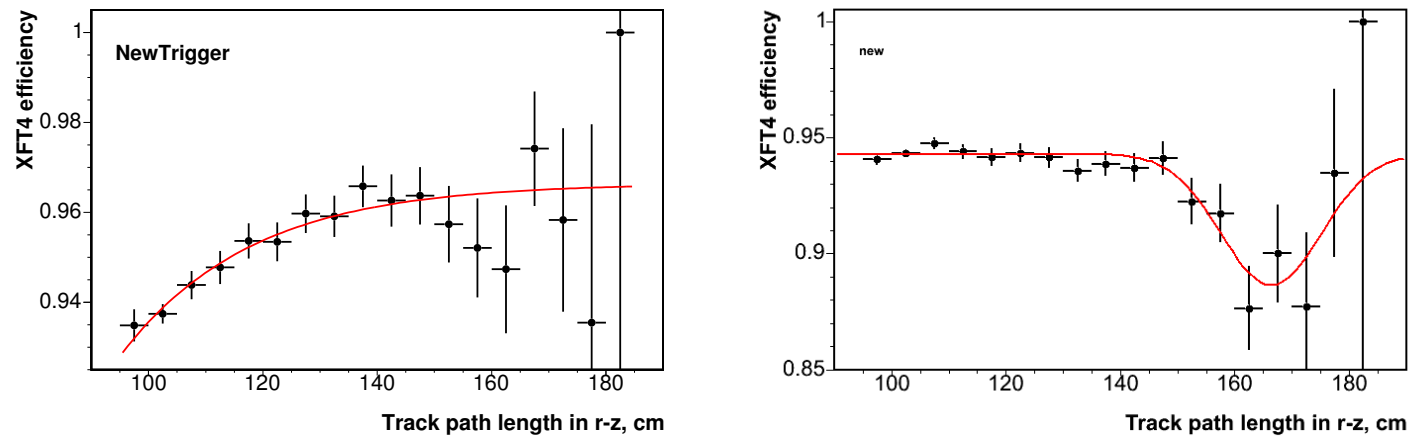

Figure 4.3: XFT track finding efficiency for tracks that do not cross the $Z=0$ plane. We parameterized this efficiency as a function of the length of the track path in the $r-z$ plane. We measure for several different periods, here we show early data 3 layer tracks (left) as well as newer data from 4 layer tracks in the COT (right). 


\begin{tabular}{|c|c|c|c|}
\hline parameter & I & III & IV \\
\hline$\epsilon_{\text {plateau }}$ & 0.9548 & 0.9539 & 0.9445 \\
\hline$A_{1}$ & 0.2646 & 0.2563 & 0.086 \\
\hline$C_{1}$ & 58.92 & 58.91 & 58.38 \\
\hline$S_{1}$ & 2.3810 & 2.3504 & 2.5063 \\
\hline$A_{2}$ & 0.3468 & 0.3320 & 0.1643 \\
\hline$C_{2}$ & 82.17 & 82.23 & 82.08 \\
\hline$S_{2}$ & 2.6352 & 2.624 & 2.413 \\
\hline$A_{3}$ & 0.4823 & 0.4604 & 0.4060 \\
\hline$C_{3}$ & 105.8 & 105.8 & 105.5 \\
\hline$S_{3}$ & 2.796 & 2.812 & 2.527 \\
\hline$A_{4}$ & 0.4457 & 0.3854 & 0.3483 \\
\hline$C_{4}$ & 131.0 & 130.8 & 129.4 \\
\hline$S_{4}$ & 3.489 & 3.695 & 2.464 \\
\hline$A_{5}$ & - & - & 70.11 \\
\hline$C_{5}$ & - & - & 2.579 \\
\hline$S_{5}$ & - & - & 0.1882 \\
\hline$A_{6}$ & - & - & 93.64 \\
\hline$C_{6}$ & - & - & 2.771 \\
\hline$S_{6}$ & - & - & 0.2933 \\
\hline$A_{7}$ & - & - & 117.3 \\
\hline$C_{7}$ & - & - & 2.980 \\
\hline$S_{7}$ & - & - & 0.4348 \\
\hline
\end{tabular}

Table 4.4: Fitted parameters for the L2 trigger efficiency as a function of $R Z 0$ (see Sub-Section 4.2.2) for tracks that cross $Z=0$.

matching in $Z \rightarrow \tau \tau$ where the tau is found with truth information. Scaled efficiency is capped at unity. This series of steps is shown in Figure 4.4. We see that efficiency is essentially $100 \%$ except for the 6 tower bin.

The same study was performed for the upgraded clustering found in category III triggers, but the clustering was found to be perfectly efficient after applying this procedure. 

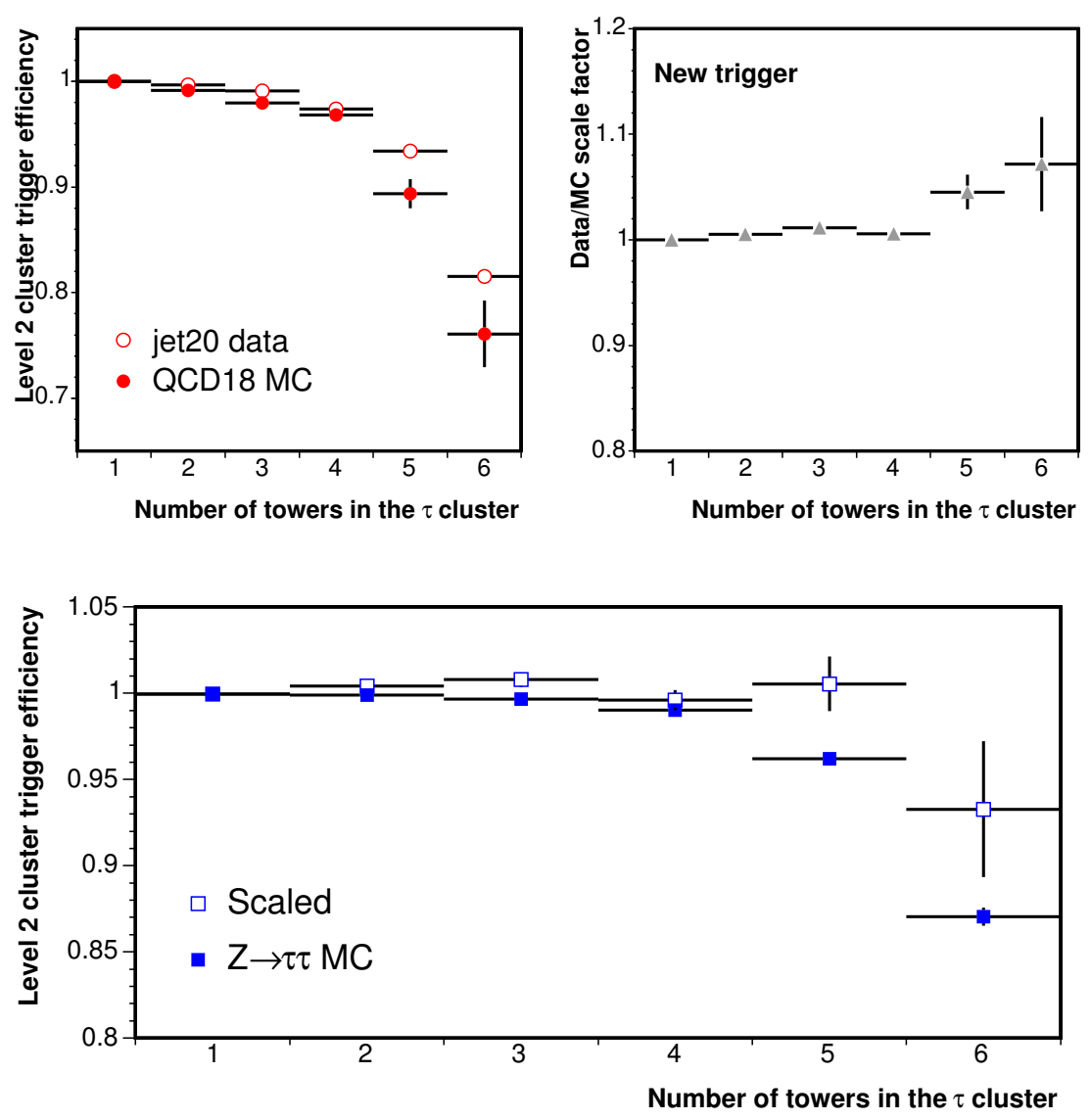

Figure 4.4: We compare the efficiency in Jet20 data and QCD $18 \mathrm{MC}$ (top left) in order to obtain a correction scale factor (top right) that we then apply to $Z \rightarrow \tau \tau$ MC efficiency (bottom). 


\begin{tabular}{c|r|r}
\hline parameter & $\mathrm{I}$ & $\mathrm{III}$ \\
\hline$\epsilon_{\text {plateau }}$ & 0.9750 & 0.9665 \\
$C$ & 1.958 & 24.63 \\
$S$ & 30.32 & 22.09 \\
\hline \hline
\end{tabular}

\begin{tabular}{c|r}
\hline parameter & $\mathrm{III}$ \\
\hline$\epsilon_{\text {plateau }}$ & 0.9448 \\
$A$ & 0.07155 \\
$C$ & 173.8 \\
$S$ & 13.59 \\
\hline
\end{tabular}

Table 4.5: Fitted parameters for the L2 trigger efficiency as a function of $\operatorname{Lr} z$ for 3 (left) and 4 (right) Layer tracks that do not cross $z=0$.

\subsubsection{Level 3 Efficiency Measurements}

Level 3 requires a tau-like isolation cone around a seed track in the COT. There are two effects that determine the trigger efficiency at Level 3. The first is the absolute normalization, which is used at the starting point for overall trigger inefficiency predominantly caused by track isolation requirements. The second is a more subtle effect, wherein inefficiency is caused by tracks being reconstructed differently offline and at Level 3. When an extra track in the signal cone is very close to the isolation cone boundary, it may end up rejected offline, leading to an inefficiency. It has been found that other tau variables like tau $P_{t}$ or $\eta$ do not effect efficiency, but goodness of track determination at L3 does.

For absolute normalization, we use a lepton environment as opposed to the jet sample. This allows us to properly quantify non-jet event effects that might infect the tau isolation cone such as tracks from the underlying event, multiple interactions or fakes. We select $Z$ events with non-isolated leptons from the high $P_{t}$ inclusive lepton trigger. Standard lepton requirements are used to select opposite-signed, sameflavored lepton pairs in the $\mathrm{Z}$ window. For each of the leptons in these events, we define all tau-like quantities around the object. For example, we construct all L3 trigger versions of a signal and isolation cone; we count the tracks and $\pi^{0}$ 's in the cones and so on. We then measure the track finding efficiency in the L3 trigger system for these leptons and parameterize the efficiency as a function of the number of vertices. We average the results between electrons and muons, and calculate for all categories of trigger (I-IV). This is the baseline efficiency that serves to normalize overall L3 efficiency, it can be found in Figures 4.5 and 4.6.

The final consideration for L3 trigger efficiency is the so-called 'track-migration' effect wherein additional tracks in the isolation cone offline migrate to the signal 


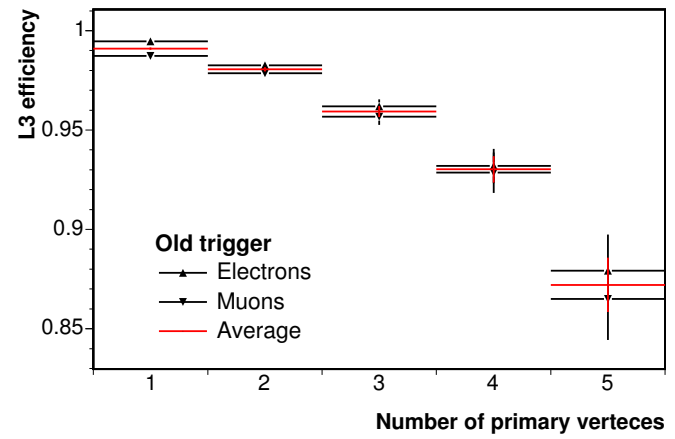

(a) Category I: No L2 requirements

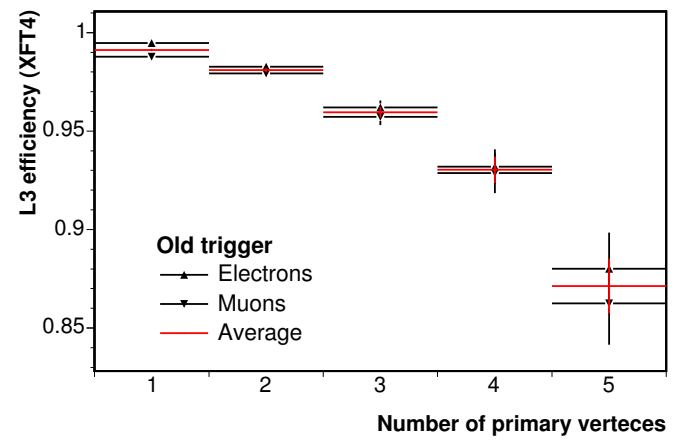

(b) Category II: XFT track matched

Figure 4.5: L3 efficiency parameterized in terms of the number of primary vertices for the old trigger.

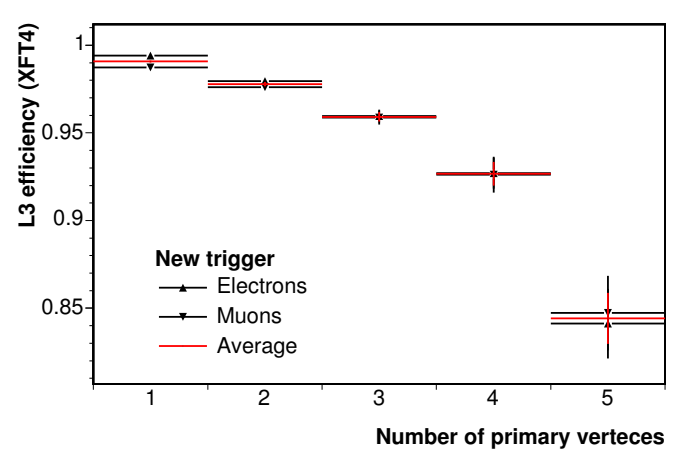

(a) Category III: New Cone and XFT track matched

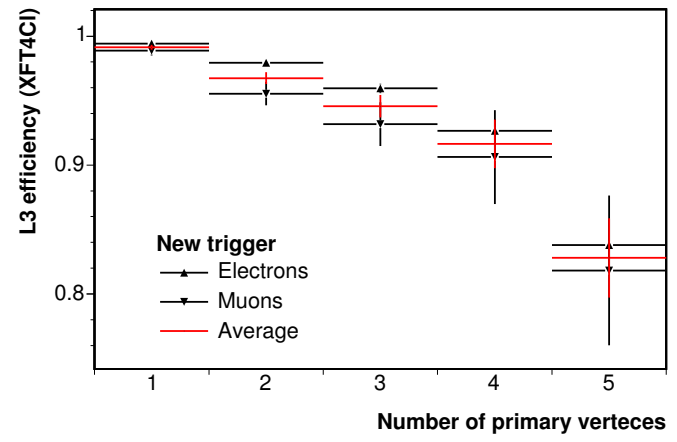

(b) Category III (electron):XFT track and L2Cluster matched

Figure 4.6: L3 efficiency parameterized in terms of the number of primary vertices for the new trigger. 
cone online. This is due to the slightly different reconstruction and variation of parameters between online and offline track reconstruction. Parameters like $P_{t}$ and $\phi$ are reconstructed well at trigger level, but less so with $\cot \theta$. The trigger efficiency drops as the additional track approaches the isolation cone.

We look at the closest extra track in the signal cone to the isolation cone and calculate the smallest $\Delta \theta$ to the boundary $\Delta \theta_{\text {min }}$. For more recent triggers (Category III and IV), we use the angle instead, and parameterize by $\Delta \alpha_{\text {min }}$. We measure the efficiency in a jet sample, which provides us with many fake taus with extra tracks, but may bias us due to differing track multiplicities between tau and jets. To avoid this bias, we only use tau candidates from a very clean environment, requiring there be no extra tracks in a cone of $\Delta R<0.7$. We again calculate the efficiency for all different categories of the triggers and we fit to a turn on function:

$$
\epsilon(x)=\frac{\epsilon_{\text {plateau }}+\epsilon_{\text {slope }} \times x}{1+e^{\frac{C-x}{S}}}
$$

The results are plotted in Figures 4.7 and 4.8 and their parameters are given in Table 4.6.

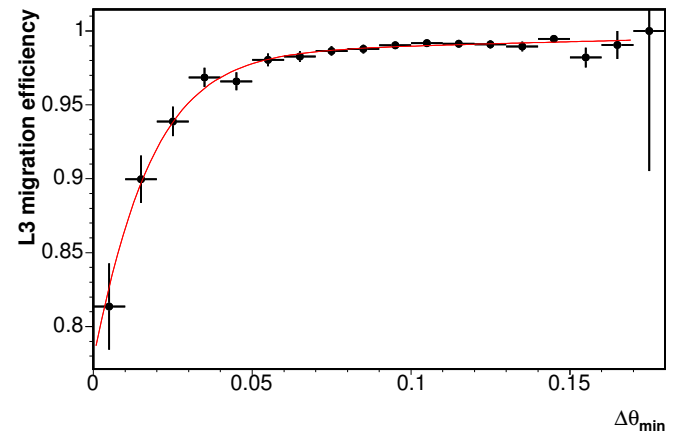

(a) No L2 requirements

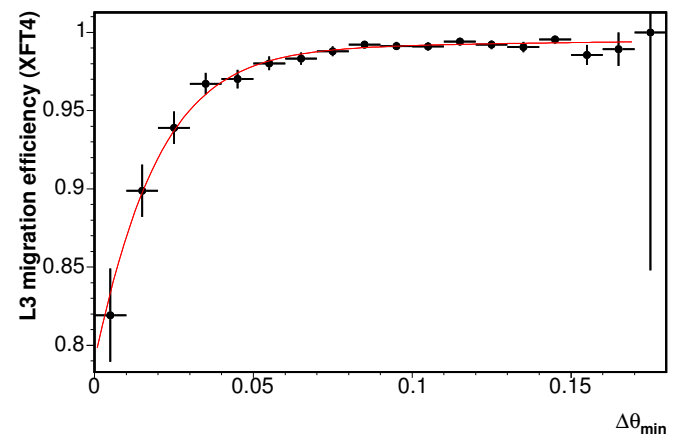

(b) XFT track matched

Figure 4.7: L3 "migration" efficiency for the Category I, II triggers, parameterized in terms of $\Delta \theta_{\text {min }}$. 


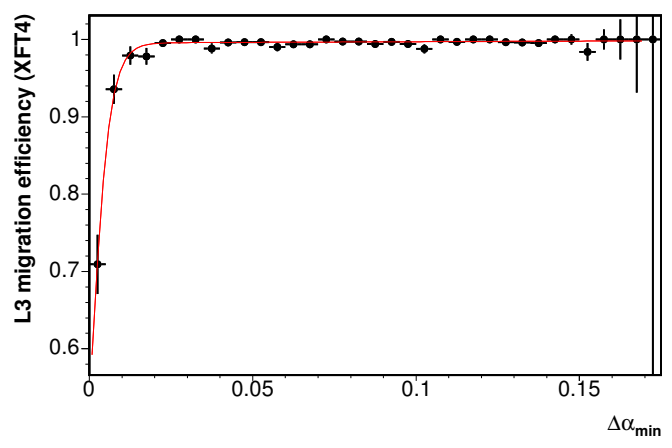

(a) XFT track matched

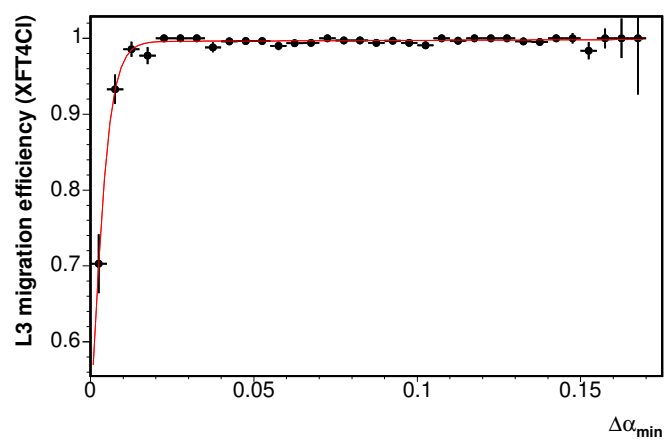

(b) XFT track and L2Cluster matched

Figure 4.8: L3 "migration" efficiency for the Category III trigger, parameterized in terms of $\Delta \alpha_{\text {min }}$

\begin{tabular}{c|rr|rr}
\hline \multirow{2}{*}{ parameter } & \multicolumn{2}{|c|}{ "Category I, II" } & \multicolumn{2}{c}{ "Category III" } \\
& no L2 & XFT & XFT & XFT+L2Cluster \\
\hline$\epsilon_{\text {plateau }}$ & 0.9848 & 0.9896 & 0.9955 & 0.9958 \\
$\epsilon_{\text {slope }}$ & $-2.011 \times 10^{-2}$ & $-2.312 \times 10^{-2}$ & $-2.991 \times 10^{-4}$ & $3.9491 \times 10^{-5}$ \\
$C$ & $1.517 \times 10^{-2}$ & $1.678 \times 10^{-2}$ & $2.985 \times 10^{-3}$ & $2.7921 \times 10^{-3}$ \\
$S$ & $5.272 \times 10^{-2}$ & $2.543 \times 10^{-2}$ & $1.598 \times 10^{-2}$ & $1.5801 \times 10^{-2}$ \\
\hline \hline
\end{tabular}

Table 4.6: Results of the fits to $\Delta \theta_{\min }$ and $\Delta \alpha_{\text {min }}$. 


\subsubsection{Note on Electron Trigger Issue}

A portion of the electron + track trigger encountered a problem for a certain period of data. Because of a trigger issue, events with $\mid \eta($ track $)-\eta(e) \mid \approx 0$ were rejected. The problem was found and fixed. Instead of trying to replicate this effect in the analysis, we simply make a cut $\mid \eta$ (track) $-\eta(e) \mid<0.3$ to cover this region of bad data. The same cut is applied to MC, weighted for the appropriate amount of luminosity. We confirm we model this effect well in various kinematic distributions.

\subsection{Lepton Selection}

We now turn to defining real physics objects as compositions of subdetector hit patterns. CDF has a long history of studying and standardizing the specific response of particles in the detector, and we use many of those standard definitions here.

The bulk of the analysis relies on a detector definition of physics lepton objects. With a few exceptions, we rely on standard CDF object definitions. Higher $P_{T}$ objects use a CDF-wide definition. Lower $P_{T}$ objects use definitions that have evolved as standard for the low- $P_{T}$ SUSY analyses at CDF.

In this section we describe the definitions of electrons and muons. We also study taus in great depth, measuring vital scale factors and corrections so we can accurately describe their interaction with the detector. Generally we will define the cuts for all objects, and then define exactly what quantities are cut on.

\subsubsection{Electron Identification}

We define an electron (CEM) as an isolated central good track in the silicon and the COT with an associated energy deposit in the EM calorimeter [37]. The cuts are as follows.

- Must have a track in the COT (Track Bit)

- The track must be fiducial to the central part of the detector (Fiducial Bit)

- $E_{T}>10 \mathrm{GeV}$ 
- $P_{T}>8 \mathrm{GeV} / c$

- $E_{h a d} / E_{E M}<0.055+0.00045 * E$

- $L_{s h r}<0.2$

- $E / P<2.0$ if $E_{T}<50 \mathrm{GeV}$

- $-3<q \Delta X_{C E S}<1.5 \mathrm{~cm}$

- $\left|\Delta Z_{C E S}\right|<3 \mathrm{~cm}$

- $\chi_{C E S s t r i p}^{2}<10$

- $\left|\Delta Z_{0}\right|<60 \mathrm{~cm}$

- $\left|\Delta d_{0}\right|<0.2 \mathrm{~cm}$

- $Z(r=\mathrm{COT})<140.0 \mathrm{~cm}$

- $>=2$ Axial SL with $>=5$ hits

- $>=3$ Stereo SL with $>=5$ hits

- $E_{\text {rel }}^{i s o}<0.1 \mathrm{GeV}$ or $E^{i s o}<2.0 \mathrm{GeV}$

- $\Sigma P_{T}^{i s o}<2.0 \mathrm{GeV} / c$ tracks in a cone of $\Delta R<0.4$

- $\mid \Delta Z_{0}-$ Event Vertex $\mid<5.0 \mathrm{~cm}$

- Electron is not tagged as a conversion

$E_{T}$ and $P_{T}$ are defined as $P_{t}=P \sin \theta$ and $E_{t}=E \sin \theta$, the transverse component of the momentum and energy 3-vector. $E_{r e l}^{i s o}$ is defined to be $E^{i s o} / E_{T}$ and therefore the isolation requirement above effectively changes at $E_{T}=20 \mathrm{GeV}$. The scale factor for these two isolation cuts have been measured and are almost exactly the same for electrons above and below $E_{T}=20 \mathrm{GeV}$.

$E_{\text {had }} / E_{E M}$ is the ratio of the energies found in the two calorimeters, electrons are expected to lose most their energy in the electromagnetic calorimeter.

$L_{s h r}$ is defined as the lateral shower tracking variable it is an energy isolation cut defined as.

$$
L_{s h r} \equiv \frac{0.14 \Sigma_{i}\left(M_{i}-P_{i}\right)}{\sqrt{\left(0.14 \sqrt{\left.E_{E} M\right)^{2}+\Sigma\left(\Delta P_{i}\right)^{2}}\right.}}
$$


The sums are over the towers in the EM cluster from reconstruction, $M_{i}$ and $P_{i}$ are the measured and predicted energies in the towers adjacent to the seed tower. $\Delta P_{i}$ is an estimate of the uncertainty on $P_{i}$.

$\Delta X_{C E S}$ and $\Delta Z_{C E S}$ are the differences between the extrapolated track in the COT and the cluster found in the CES.

$\chi_{C E S s t r i p}^{2}$ is a $\chi^{2}$ fit between an ideal electron shower profile and the measured shower profile in the event.

$\Delta Z_{0}$ and $\Delta d_{0}$ are the distances of track closest approach from detector center in $\mathrm{Z}$ and $\mathrm{r}$ respectively.

$Z(r=\mathrm{COT})$ is a fiducial requirement ensuring the electron is contained in the full length of the COT.

The Axial and Stereo Superlayer (SL) requirements mandate at least 5 hits in a superlayer with at least a certain number of SLs to make a track.

The track isolation cut is a non-standard cut, and as such, we must calculate its effect and corresponding reconstruction and identification scale factor. This has been done by looking at $Z \rightarrow e e$ samples in both MC and data. The scale factor is measured to be $0.997 \pm 0.001$ indicating $\mathrm{MC}$ does a good job describing the track isolation cut. This scale factor is shown to be stable with electron $E_{T}$.

Conversion removal is done with the standard CDF method with $\left(\left|S_{X Y}\right| \equiv D_{X Y}\right)<$ $0.2 \mathrm{~cm}$ and $(|\Delta \lambda| \equiv \Delta \cot \theta)<0.04$. The efficiency for this cut in data is measured to be $83.8 \% \pm 0.3 \%$. The scale factor for total reconstruction and identification is applied to $\mathrm{MC}$ is measured as standard CDF electrons. We use a single luminosity scale factor weighted per-period, and apply it to every good electron found in every MC sample used in the analysis. For this reason, it is difficult to break the analysis up into smaller data subsamples.

\subsubsection{Muon Identification}

We define muons similarly to electrons, but we require deposits in both calorimeters consistent with muon energy deposits [38]. We also require hits, or stubs in separate muon detectors. These detectors define the muon type. We use muons that have hits in both the overlapping CMU and CMP chambers, we call these CMUP muons. 
We also use muons coming from the CMX chambers. The requirements differ more dramatically than electrons for objects below the $P_{T}=20 \mathrm{GeV} / c$ threshold. The requirements are as follows. We define quantities not common to electrons in section 4.3.1.

- $P_{T}>10 \mathrm{GeV} / c$ for all Muons

- $\left|\Delta Z_{0}\right|<60 \mathrm{~cm}$

- $\left|\Delta d_{0}\right|<0.2 \mathrm{~cm}$

- $Z(r=\mathrm{COT})<140.0 \mathrm{~cm}$

- $>=2$ Axial SL with $>=5$ hits

- $>=3$ Stereo SL with $>=5$ hits

- $E_{\text {rel }}^{i s o}<0.1 \mathrm{GeV}$ or $E^{i s o}<2.0 \mathrm{GeV}$

- $\Sigma P_{T}^{i s o}<2.0 \mathrm{GeV} / \mathrm{c}$ tracks in a cone of $\Delta R<0.4$

- $\mid \Delta Z_{0}-$ Event Vertex $\mid<5.0 \mathrm{~cm}$

$\underline{P_{t}>20 \mathrm{GeV} / c}$

- $E_{E M}<2+\max (0,0.0115 *(p-100)) \mathrm{GeV}$

- $E_{H A D}<6+\max (0,0.028 *(p-100)) \mathrm{GeV}$

- $\left|\Delta X_{C M U}\right|<3 \mathrm{~cm}$ and $\left|\Delta X_{C M P}\right|<7 \mathrm{~cm}$ (CMUP type)

- $\left|\Delta X_{C M X}\right|<6 \mathrm{~cm}$ (CMX type)

$\underline{P_{t}<20 \mathrm{GeV} / c}$

- $E_{E M}<2 \mathrm{GeV}$

- $E_{H A D}<3.5+\left(P_{t} / 8.0\right) \mathrm{GeV} / c$

- $\left(\left|\Delta X_{C M U}\right|<3 \mathrm{~cm}\right.$ or $\left.\chi_{C M U}^{2}<9.0\right)$ and $\left(\left|\Delta X_{C M P}\right|<7 \mathrm{~cm}\right.$ or $\left.\chi_{C M U}^{2}<9.0\right)$ (CMUP type)

- $\left|\Delta X_{C M X}\right|<6 \mathrm{~cm}$ or $\chi_{C M X}^{2}<9.0$ (CMX type) 
The track isolation requirement $\Sigma P_{T}^{i s o}<2.0 \mathrm{GeV} / c$ is measured as in section 4.3.1 and found to contribute a scale factor of $1.00 \pm 0.001$, again without any $P_{T}$ dependence.

The $\Delta X_{C M(U, P, X)}$ quantities are the differences between the extrapolated track and the muon stub, or hit, in the respective muon detectors. As can be seen, we have different requirements for high and low $P_{T}$ muons. Low $P_{t}$ muons can also be accepted based on a good track $\chi^{2}$ which is a track goodness of fit between the hits in the COT and the extrapolated track.

We use the same method to calculate the luminosity weighted reconstruction and ID scale factors for the different muon types using a combination of sources. For muons over $P_{T}=20 \mathrm{GeV} / c$ we simply use standard CDF numbers. For CMUP and CMX we get $.945 \pm .009$ and $.976 \pm .01$ respectively for the $6.0 \mathrm{fb}^{-1}$ data sample we use in this analysis. For muons below the $P_{T}=20 \mathrm{GeV} / c$ threshold, we use numbers that have been calculated previously from separate SUSY analysis that gives a scale factor of .909 \pm .001 for CMUP muons and gives .892 \pm .034 for our CMX muons.

\subsubsection{Tau Definition}

The definition of a hadronic tau lepton decay is by far the most complicated object in this analysis. Our ability to identify real taus, and to differentiate them from QCD jets determines our signal sensitivity.

We aim to reconstruct all 1 and 3 pronged hadronic decay modes of the tau using the visible decay products. The definition of the tau has evolved at CDF [39-42] , and we use the most current and common definition, along with the associated scale factors.

We define two types of tau leptons, loose and tight; the tight tau is a subset of the loose. The final selection in our data will be tight taus, and our background will be determined by a method using loose taus, which are mostly QCD jets faking real taus as described in section 5.2.1. The loose tau is the loosest fiducial object we can define that is tighter than our trigger object. 


\subsubsection{Tau Quantities}

Because of the uniqueness of hadronic taus, we need to specifically define certain quantities associated with taus that are non-obvious.

The four-momentum of taus is defined here as the sum of the tracks and $\pi^{0} \mathrm{~s}$ in the cone that makes up the tau, see Figure 4.9. We use this same momentum vector to determine the mass of the tau, namely $M\left(\right.$ tracks $\left.+\pi^{0} s\right)$. There may be energy loss if we miss a $\pi^{0}$ in the detector, so we must apply an energy correction to reconstructed taus.

We correct energies using both the sum of the tracks and $\pi^{0} \mathrm{~s}$ and the energy deposited in the tau calorimeter cluster. If the energy of the tracks and $\pi^{0} \mathrm{~s}$ are greater that the calorimeter, we simply use the tracks and $\pi^{0} \mathrm{~s}$, otherwise we check to see if we have significant hadronic contributions. This is true if $E_{C L U S T E R}-P_{T R A C K S}>3 * \sigma_{\text {had }}$ where $\sigma_{\text {had }}=0.5 * \sqrt{E_{C L U S T E R} *(1-\mathrm{emfr})}$ where emfr is the fraction of the total energy that is electromagnetic. If this is true, we correct the energy of the tau by using the cluster energy in lieu of the tracks and $\pi^{0} \mathrm{~s}$.

We have a cut on the tau to reduce electrons and muons with large deposits of EM energy faking a tau,

$$
\xi^{\prime}=\frac{E_{t o t}}{\sum|\boldsymbol{p}|}\left(0.95-\frac{E_{E M}}{E_{t o t}}\right)
$$

where $\boldsymbol{p}$ is the momentum of the charged tracks in the tau, $E_{t o t}$ is the total calorimeter energy and $E_{E M}$ is the electromagnetic component of the calorimeter energy.

The tau is defined by a signal cone and an isolation cone as in Fig. 4.9. Unlike QCD jets, a real tau should have activity in the signal cone, but not in the isolation cone. Our signal cone is defined as $\theta_{\text {sig }}=\min \left(0.17, \frac{5.0 \mathrm{rad} / \mathrm{GeV}}{E^{\tau c l}}\right)$. We prevent the cone from getting too small by setting a minimum cone size of $0.05<\theta_{\text {sig }}$ rad for tracks and $0.1<\theta_{\text {sig }}$ rad for $\pi^{0}$ candidates. The isolation cone, $\theta_{\text {iso }}$ is defined as $0.52 \mathrm{rad}$ excluding the enclosed signal cone. To quantify the activity in the isolation cone we use the sum of the $P_{T}$ of the tracks in the isolation cone $\Sigma P_{T, t r k}^{i s o}$ and the sum of the $E_{T}$ of the neutral pions in the isolation cone $P_{T, \pi^{0}}^{i s o}$.

Besides the cone, we construct a tau using energy deposited in the calorimeters. 
We have the energy of a seed tower, $E_{T}^{\text {seedtwr }}$ as well as surrounding "shoulder" towers $E_{T}^{\text {shtwr }}$. Similarly we have seed tracks $P_{T}^{\text {seedtrk }}$ and shoulder tracks $P_{T}^{\text {shtrk }}$.

The cuts for our tau candidates are as follows.

- $E_{T}^{\text {seedtwr }}>6.0 \mathrm{GeV} *$

- $E_{T}^{\text {shtwr }}>1.0 \mathrm{GeV}^{*}$

- $N^{t w r} \leq 6 *$

- $P_{T}^{\text {seedtrk }}>6.0 \mathrm{GeV} / c$

- $P_{T}^{\text {shtrk }}>1.0 \mathrm{GeV} / c$

- $E_{T}^{\tau c l}>9.0 \mathrm{GeV}$

- $P_{T}>15.0 \mathrm{GeV} / c$ for 1 -pronged, $P_{T}>20.0 \mathrm{GeV} / c$ for 3 -pronged

- $\Delta Z^{\text {shtrk }}<5.0 \mathrm{~cm}$

- $\Sigma P_{T, t r k}^{i s o}<2.0 \mathrm{GeV} / c$, and no tracks with $P_{t}>1.5 \mathrm{GeV} / c^{*}$

- $\Sigma E_{T, \pi^{0}}^{i s o}<1.0 \mathrm{GeV}^{*}$

- $N_{\text {sig }}^{\text {trk }}=1,3$

- $\left|\Sigma Q^{t r k}\right|=1$

- $M\left(\operatorname{trks}+\pi^{0} s\right)<1.8 \mathrm{GeV} / c^{2} *$

- $\xi^{\prime}>0.1^{*}$

- Fiducial to all axial SLs of the COT

* Denotes cuts specific to a tight tau.

We suppress electrons accompanied by bremsstrahlung that could fake a tau. We do this by rejecting 1-prong tau candidates if a $\pi^{0}$ with $\left|\Delta Z_{C E S}<2.0\right| \mathrm{cm}$ from the projection of the track, and if $\phi$ lies between the CES intersect of the track helix and its tangential. To account for CES position uncertainty, and track extrapolation resolution, the veto region is extended by 0.01 radians beyond the point the track extrapolates to the CES. 


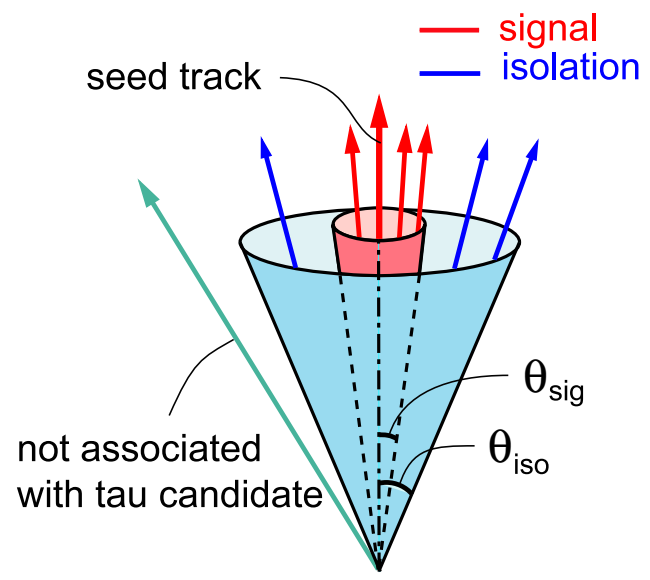

Figure 4.9: Tau isolation and signal cones.

There is one additional cut used only in events containing an electron wherein for 1-pronged taus we require fiduciality in the CES. We require $\left|x_{C E S}\right|<21 \mathrm{~cm}$ since our simulation has deficiencies in regions near $\phi$ cracks, and this cut restricts the track to the fiducial region.

\subsubsection{Tau Scale Factors and Systematics}

We calculate the scale factors to be used for tau MC. Specifically, the isolation cone and the ability to accurately reproduce the $\pi^{0}$ and track activity must be measured. This is done by measuring events that have a similar environment to our event selection. We select $Z \rightarrow e e$ and $Z \rightarrow \mu \mu$ events and implement an isolation requirement for one lepton. As an initial selection, we use an isolation cone between $10^{\circ}-30^{\circ}$ of the track, but the slight difference in isolation definition should be irrelevant both isolation cones describe the same environment. We select events with standard $Z$ selection criteria, opposite-signed electrons and muons are selected with all tight cuts, except for isolation. Events with an invariant mass between $66<M(l l)<116$ $\mathrm{GeV} / c^{2}$ are selected.

With these events, we measure three types of isolation. First we replicate the Level 3 trigger's isolation and measure our efficiency of doing so, $\epsilon^{\text {L3iso }}$. Then we replicate the efficiency of the tau isolation cuts, $\Sigma E_{T, \pi^{0}}^{i s o}<1.0 \mathrm{GeV}$, and $\Sigma P_{T, t r k}^{i s o}<2.0$ $\mathrm{GeV} / c$ and measure the efficiency of these cuts, $\epsilon^{i s o}$.

As described in section 4.2 we have two substantively different trigger periods when 
it comes to isolation we refer to them as the "old trigger" and the "new trigger". The scale factor for the offline replication of the L3 requirements, $\epsilon_{\text {data }}^{L 3 i s o} / \epsilon_{M C}^{L 3 i s o}$ is found to be $0.997 \pm 0.001$ for the old trigger and $0.999 \pm 0.001$ for the new trigger. We then turn to the analysis level isolation cuts. We find the efficiency is dependent on the number of vertices in the event or, correspondingly, the instantaneous luminosity. This makes sense, as high luminosity events contain more activity, isolation cuts suffer in efficiency. For simplification, we integrate over all vertices and measure $\epsilon_{\text {data }}^{\text {iso }} / \epsilon_{M C}^{i s o}$ to be $0.989 \pm 0.001$ for the old trigger and $0.984 \pm 0.001$ for the new.

We also observe an effect called "multiplicity migration" wherein $P_{T}>1.0 \mathrm{GeV}$ tracks from the underlying event are within the $10^{\circ}$ signal cone of the tau. Measuring the additional tracks in the data, we find $0.025 \pm 0.013$ additional on average, and in $\mathrm{MC}$ we find $0.027 \pm 0.003$. This "multiplicity migration" therefore corresponds to a $0.3 \%$ uncertainty on tau selection.

From previous studies, we have found a hadronic scale uncertainty on the tau selection in the MC due to cuts on tau cluster energy, seed tower $E_{T}$ and $\xi^{\prime}$. Previously, we have found an uncertainty of $2 \%$ which is what we use here.

There are additional substantial effects on taus selection due to the $M(t r k s+$ $\left.\pi^{0} s\right)<1.8 \mathrm{GeV} / c^{2}$ cut. We examine the effect of this cut in a separate data sample of $W$ decaying to real taus and missing energy selected with the 'tau $+\mathbb{E}_{T}$ ' trigger. In this body of data, identical tau id is applied, except for accommodating trigger thresholds by raising the seed track $P_{T}$ cut to $P_{T}^{\text {seed }}>10 \mathrm{GeV} / c$. To select clean $W$ events $E_{T}>30 \mathrm{GeV}$ is required and no jets with an $E_{T}>5.0 \mathrm{GeV}$ are allowed in the event. This jet requirement introduces a dependence on the jet modeling which complicates exact determination of tau yield. Since tau yield is not needed, we simply fit the backgrounds to the data. This is done by constraining the MC used to its cross section, and floating the QCD model in the fit. The QCD model used is jet to tau fakes that enter the sample and is taken from templates formed by data from the hadronic jet trigger. With this model now in place, it is simple to cut on $M\left(\operatorname{trks}+\pi^{0} s\right)<1.8 \mathrm{GeV} / c^{2}$ to find the scale factor. As reported, the scale factor is $\epsilon_{\text {data }}^{m} / \epsilon_{M C}^{m}=\frac{0.952 \pm 0.003}{0.969 \pm .003}=0.982 \pm 0.05$. Unlike previous analysis, we do not relax the tau mass cut for 3 pronged taus.

The combination of the above systematics requires a total $3 \%$ systematic on the uncertainty of hadronically decaying taus. Every MC event with a hadronically decaying tau is scaled by the factors above. 


\subsubsection{Tau Energy Scale}

Reconstructing the tau energy accurately is much more difficult than electrons and muons due to the presence of neutrinos in the decay as well as the diffuse nature of tau decays. We measure the effect using the same sample of $W \rightarrow \tau \nu$ events modeled in section 4.3.3.2. The $P_{T}$ of tau candidates is compared between data and MC for both 1 and 3 pronged taus. The consistency of the $P_{T}$ shape distribution is compared with a Kolmogorov-Smirnov test of shape consistency while shifting the energy scale of the MC events. The best agreement is achieved by shifting the data by $+1 \%$ for 1 pronged taus and $-0.5 \%$ for 3 pronged taus. A $1 \%$ systematic on the tau data/MC energy scale is added to account for this effect.

\subsubsection{Jet Definition}

Generally jets originate from hadronic particles and decay immediately to a spray of multiple objects. In the detector, jets look like a deposit of EM energy correlated with a many tracks. To reconstruct jets, we identify them with a standard (JETCLU) algorithm in cones of $\Delta R=0.7$. After the jets are clustered, they are corrected to 'Level 5', which includes corrections for detector calibrations, and detector geometry. This Jet Energy Correction (JEC) will lead to a systematic error due to its influence on the total energy of the event. We select events with a corrected $E_{T}>8 \mathrm{GeV}$ and an EM fraction of $<0.9$. Generally we do not use jets for analysis objects, but identify them for $\mathbb{E}_{T}$ corrections.

\subsection{5 $\quad \ddot{H}_{T}$ Definition}

Neutrinos and, possibly some SUSY particles do not interact with the detector, and therefore carry away energy and momentum, leaving a net imbalance. Because we cannot determine the net initial longitudinal energy of the interacting quarks, we must restrict ourselves to the transverse component when defining $\mathbb{E}_{T}$. $\mathbb{E}_{T}$ is defined as:

$$
E_{T} \equiv-\Sigma_{i=1}^{N} E_{\text {toweri }} \sin \theta_{i} \boldsymbol{n}_{i}
$$

Where $N$ is the total number of EM towers, $i$ is the individual tower and $\boldsymbol{n}_{i}$ is 
the normal of the particular tower.

Corrections for identified physics objects are applied to this generic $\mathbb{E}_{T}$ quantity. When the object can determine the energy better than the generic tower sum, we use the better quantity. So, for example, $\not_{T}$ is corrected for muons for the difference between the well measured muon track $P_{T}$ and $E_{T} \equiv \frac{E_{e m}+E_{h a d}}{\cosh \eta}$. In this analysis, $\mathbb{E}_{T}$ is corrected for electrons, muons, taus, and jets.

\subsubsection{Cosmic Veto}

We veto events containing muons from cosmic rays. When energetic particles interact with Earth's atmosphere, they produce a shower of pions and kaons. These particles then decay into muons and can reach the surface of the Earth and observed in the detector. We use the Cosmic Ray Tagger which looks for events that form back-toback tracks that are out of time with collisions and veto these events, the efficiency of this cut is $99.9 \%$. 


\section{Chapter 5}

\section{Background Model}

In order to discover new SUSY particles with the signature of the lepton + tau channel in our detector we must model well its response to the standard model particles. We develop a background model composed of the complete set of standard model processes that will resemble our signal. These backgrounds can be broken down into two broad categories: those that contain a fake tau, and those that do not. By far, the dominant background of the analysis will be hadronic jets from strong production processes faking the signature of a tau lepton accompanied by a real lepton in the event. Other standard model process with real taus, or with leptons faking taus, will be a small background in our signal region. However these processes will dominate in our OS control region.

In this chapter, I describe the model we use for standard model processes with real taus or lepton faking tau events. I then describe in detail our data-based method that accounts for jet-to-tau fakes. This includes the method, measuring the fake rate, and validating the result in orthogonal regions of data.

In the following chapter we look at plots of the OS control regions, which give us confidence in our MC background method as well as additional confirmation of the fake rate method. Only at that point do we have confidence enough to examine the data in the SS region. 


\subsection{Monte Carlo Backgrounds}

We use MC simulation to model detector response to all MC processes without a jet faking tau. This includes processes a real lepton fakes a tau. We also assume that events wherein objects fake a lepton are simulated by MC. Even to the extent these events are not well modeled by MC, they contribute several orders of magnitude less to the background than the jet-to-tau fake rate.

The following is a list of the processes that are modeled by MC:

- $Z \rightarrow e e, Z \rightarrow \mu \mu, Z \rightarrow \tau \tau: Z$ boson production with decay to leptons. In the case of $Z \rightarrow e e$ and $Z \rightarrow \mu \mu$, one lepton fakes a tau. In the case of $Z \rightarrow \tau \tau$ one tau decays to a lepton and the other is a real tau. In this case, the charge of one of the legs must be mis-reconstructed to appear in the SS signal region. This will be the dominant process in the OS control region.

- $\left(W \rightarrow \tau \nu_{\tau}\right)+$ Jets: The $W$ decays to a real tau, and the jet (or other event activity) fakes a lepton.

- $t \bar{t}$ : The top quark always decays to a bottom quark and a $W$ boson. The combinations of final states vary, but can generally produce an all hadronic final state, a final state with real leptons and hadronic jets, or a dilepton final state. This process has a very small cross section.

- Diboson: $Z Z, W Z$, and $W W$ production can all decay to real leptons, including taus, that mimic the signal. Like $t \bar{t}$ these processes have a small cross section $(\sigma \approx 1 \mathrm{pb})$.

These processes are normalized to their theoretical SM cross sections. A systematic uncertainty on the cross section is applied in accordance with each processes uncertainty. A k-factor is applied to account for the NLO cross section.

\subsection{Tau Fake Rate Backgrounds}

The dominant background for this analysis are processes wherein a QCD jet fakes the hadronic decay of a tau lepton. Because of the similarities between jets and taus, 
the rate at which jets fake a tau can be several orders of magnitude higher than the rate at which jets fake an electron or muon.

We use a method developed in previous CDF searches to measure this fake rate in a sample of QCD jets. Using this measurement in the QCD sample, we turn to our analysis dataset and look at all the jets in every event with an associated lepton. We will carry each jet through our analysis as if it were a real tau, making all the appropriate event level cuts. If the jet survives these cuts, we apply the appropriately parameterized fake rate from our measurement as a weight to the event. These weighted events are then classified as our jet-to-tau fake rate background. The method we use accounts for all processes in which a jet faking tau can enter our final sample, we do not try to account for these processes individually. To enter our final sample, a process must contain a lepton (electron or muon) and a jet capable of faking the tau. We call this jet a loose tau. The processes that fall into this category are as follows.

1. $\mathbf{W}+$ Jets $\mathrm{A} \mathrm{W}$ boson is produced with associated jets. The $\mathrm{W}$ decays to an electron or muon and a neutrino and the jet fakes a hadronic tau. This process has a very high cross section a the Tevatron. Because the neutrino escapes the detector as missing energy, $\mathrm{W}+$ Jets is a dominant background of the analysis. It will tend to have significant missing energy, a real lepton, and jets that fake our tau.

2. $\gamma+$ Jets The photon in $\gamma+$ jet events can interact with the material in the detector and convert to an electron - positron pair. We then pick up the real electron or positron, and the associated jet fakes our hadronic tau. Although we do reject electrons and positrons that come from conversions, normally one of the legs of the conversion is soft and may go undetected. Therefore this conversion veto is only $83.8 \% \pm 0.3 \%$ efficient.

3. $\boldsymbol{p} \overline{\boldsymbol{p}} \rightarrow \mathbf{N}$ Jets Due to the extremely high QCD multi-jet cross section, we can have one jet fake an electron or muon and another jet fake our hadronic tau. Although the QCD rate will be large, it should have a different kinematic shape from our signal, namely low missing energy, and therefore be reducible in our final selection. 


\subsubsection{Jet-to- $\tau$ Fake Rate Measurement}

We must measure the rate at which loose taus fake tight taus so it may be applied to the analysis data sample for an accurate estimate of jets polluting our final selection.

The measurement strategy is as follows. We measure the rate in a sample rich in QCD jets and independent of our analysis dataset. We reconstruct the jets as tight and loose taus to determine what percentage of loose taus successfully fake a tight tau. The fake rate then is the ratio of tight taus over loose. The measurement of this fake rate is described here, and the intricacies of its application is described more fully later in the text, Section 5.2.3.

Because of the tau-like requirements of the track side of our lepton + track trigger, we need a tighter definition of a jet than the standard CDF Jet Cone. Our jet definition must be tighter than the trigger, so we can apply the fake rate directly to our triggered data without losing fake candidates cut by the trigger. We would, however, like our denominator object to be as loose as possible, since the difference between the numerator (tight tau) and denominator (loose tau) determines the statistics of the events used in the background determination. Since the events contributing to this background estimate will have a tau with purity between the tight and loose object, the closer the definitions of the two types of objects, the fewer statistics we will have. This loose tau will be tighter than the trigger requirements, but otherwise as loose as possible. We therefore define a loose tau as our fakeable jet denominator, see section 4.3.3 for the definition. Our standard tight tau will be the numerator object. It is important to note that in defining these fake rates, we restrict their use to data coming from the lepton + track trigger since other triggers may have looser denominator objects.

The jet-rich sample used at CDF is triggered on generic jets at different $E_{t}$ thresholds. These datasets consist of jet events triggered at an $E_{T}$ of $5 \mathrm{GeV}$ (ST5), $20 \mathrm{GeV}$ (JET 20), $50 \mathrm{GeV}$ (JET 50), $70 \mathrm{GeV}$ (JET 70) and $100 \mathrm{GeV}$ (Jet 100). The different thresholds are needed to mitigate the effect of trigger prescale on the data; lower $E_{T}$ jets of $5 \mathrm{GeV}$ are so common the trigger needs to be pre-scaled up to factors of 50,000 whereas the JET 100 trigger needs no prescale. Because these trigger paths overlap, high $E_{T}$ jets are triggered on all trigger paths. We classify individual events only by their highest $E_{T}$ trigger; this eliminates the possibility an event may be used more than once in our measurement. The leading and sub-leading jet are used as a high and low fake rate estimate respectively. It has been observed previously and it will 
be shown below in section 5.2.2 that this serves as a good estimate of the data. In the application of the fake rate, the average of the two measurements is used.

After numerous studies, previous analyses have pinpointed the most efficient fake rate parameterization $[40,42]$. We parameterize in terms of the number of tracks in the tau cone; $E_{T}^{c l}+E_{T}^{c a l i s o}$ the sum of the energies in the tau isolation cone and the tau cone; and $\eta_{\text {det }}$, the $\eta$ at which the tau exits the detector. A sample of these relative fake rates is shown in Figure 5.1. Note that at low transverse energies, the fake rate can be quite large, up to $\approx 40 \%$; which is why tau fakes make such a significant contribution.

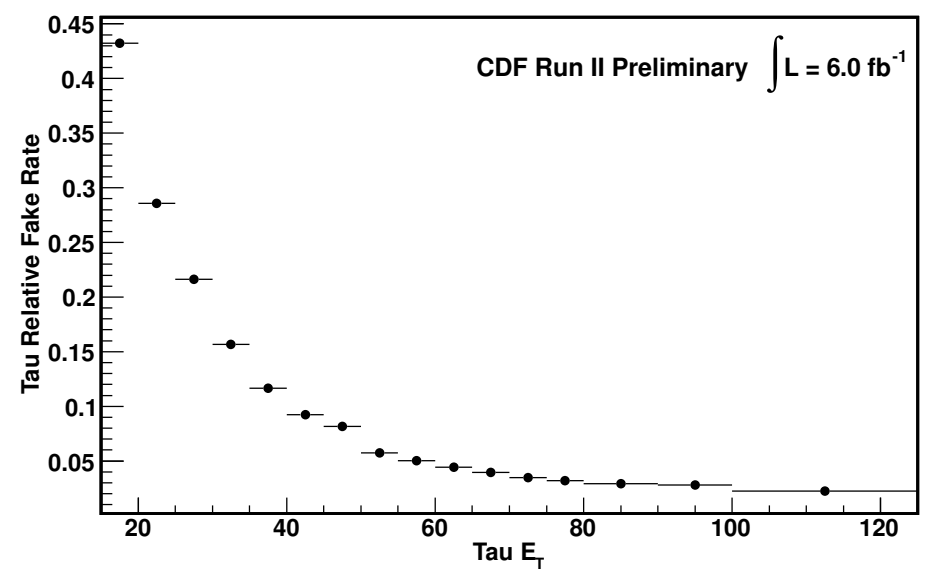

Figure 5.1: Tau Relative Fake Rates. For simplicity, these particular fake rates are calculated with any jet in the event, for any number of tracks, and for all $\eta_{\text {det }}$. The used fake rates are parameterized as described in the text.

In section 5.2.3 we will see that in order to properly apply these fake rates, we will need a measurement of the efficiency of finding a tight tau. Because there is no suitable data-driven method to do this, we must use Monte Carlo. We look at the Monte Carlo truth information to find the real tau, and then we simply determine the rate at which we reconstruct these taus after full detector simulation.

\subsubsection{Jet-to- $\tau$ Fake Rate Verification}

Since tau fakes are the dominant background in the analysis, it is vital we validate these rates in a sample orthogonal to our signal sample. We identify three distinct samples to test predicted fakes against observed tight taus. All the samples necessarily 
come from our trigger path, and represent unique physical processes to account for possible jet shape differences. All samples are fully carried through the analysis as normal with the exception of the noted cut alterations.

1. Non-isolated leptons The isolation selection of the electron/muon leg is altered by requiring activity in the isolation cone. Specifically, for electrons and muons, EM activity is required $3 \mathrm{GeV}<E_{\text {cal }}^{i s o}<20 \mathrm{GeV}$. Track isolation is also reversed so $3 \mathrm{GeV}<E_{T}^{i s o}<20 \mathrm{GeV}$. Isolation is not directly inverted to provide some separation from production cuts, creating a more distinct sample. These events should mimic a multi-jet, QCD like sample.

2. $\mathbf{W}+$ Jets In this sample, we select events in a $\mathrm{W}+$ Jets rich environment. We use a 2 dimensional $\zeta$ cut to include processes that do not have kinematics similar to processes coming from 2 body decays without $\mathbb{E}_{T}$, see for a description of the cut. This cut will tend to veto $\mathrm{Z}$ boson production and enhance $\mathrm{W}+$ Jet production. This region is similar to our signal region, so we use opposite signed events to keep the verification orthogonal to our selection.

3. $\gamma+$ Jets For the electron channel only, we select the $\gamma+$ Jet process by explicitly requiring a conversion in the event. We simply invert the conversion veto on the electron, we assume that these tagged conversions come from $\gamma+$ Jets processes.

We develop a high and low banded prediction of the fake rate, and compare the results to what is seen in data. The band is the estimated fake rate range, which is identified by selecting fake candidates (loose taus) in the sample. We then apply to these loose taus the high and low measured fake rate according to our parameterization. The high range is the fake rate calculated with the leading jet in the fake rate measurement, and the low range is the rate measured for the subleading jet. Remember, for the application of the fake rates in the analysis, we apply the average of these two rates. Thus, we expect the majority of the data to fall between the bands formed by the two measurements. These data points are simply events with identified tight taus.

This verification method is valid under two assumptions. Firstly, that the process examined is dominated by fake taus, which we have assured by selecting jet-rich processes. As a precaution however, we use the correction procedure for tight taus 
as described in Section 5.2.3. Second, we assume the process has minimal standard model contributions from other processes, which we have confirmed with Monte Carlo.

We plot several distributions for every sample, some of which are included below. We do not expect the shapes of the distributions to resemble the shapes of the final analysis as we are modeling distinct processes.

We see that the data falls within the expected region in most all these samples and regions giving us confidence in our measurement. As the systematic uncertainty for this method we take as the half difference between the bands formed by this check.

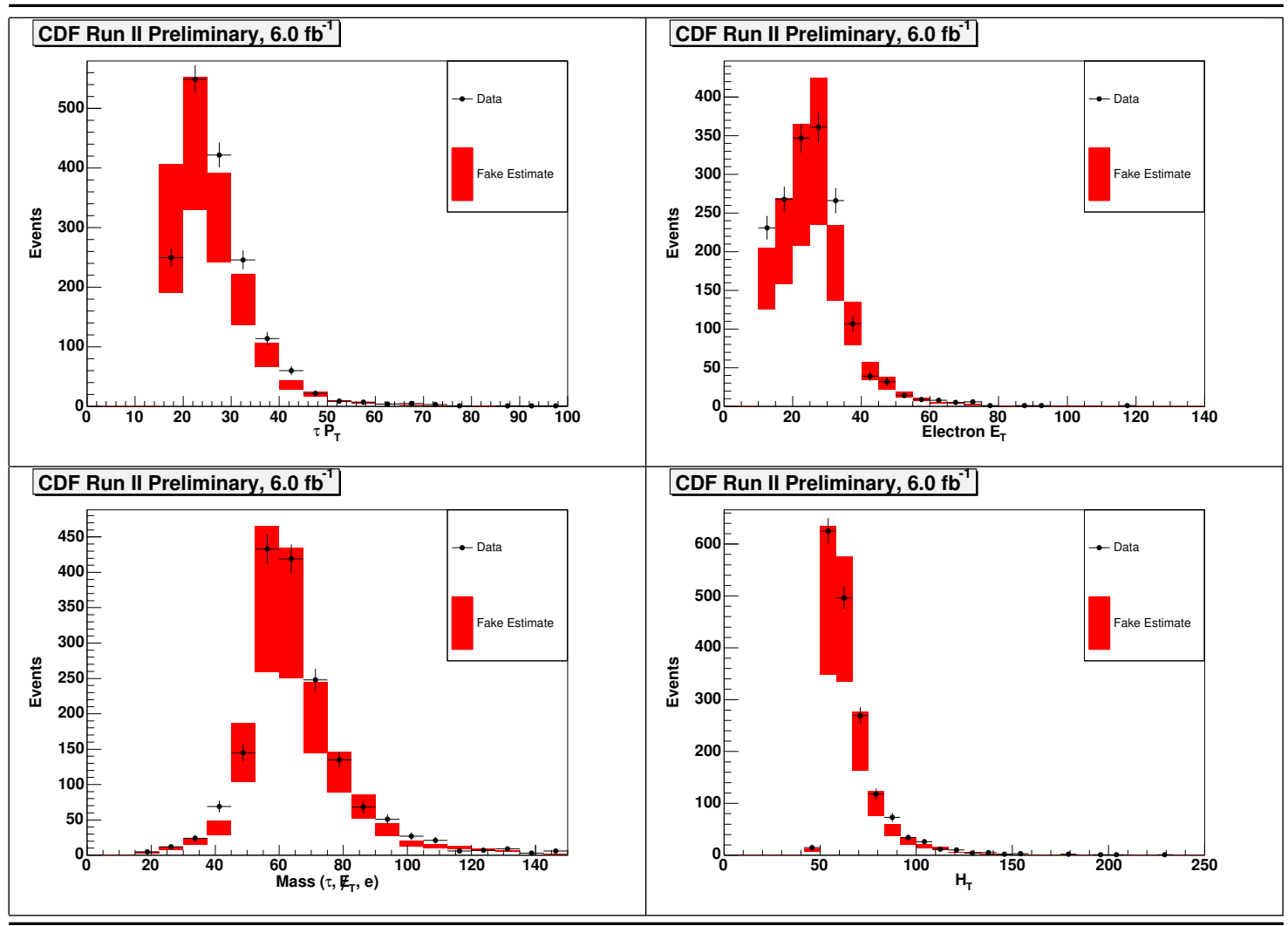

Figure 5.2: Fake Rate Verification. Conversion tagged electron events. Bands formed by leading and sub-leading jets in fake rate measurement. Data points are from tight tau identification.

\subsubsection{Jet-to- $\tau$ Fake Rate Application}

To apply the measured fake rates, we select events with an electron or muon and a loose tau and carry these taus through the analysis exactly as if they were real 


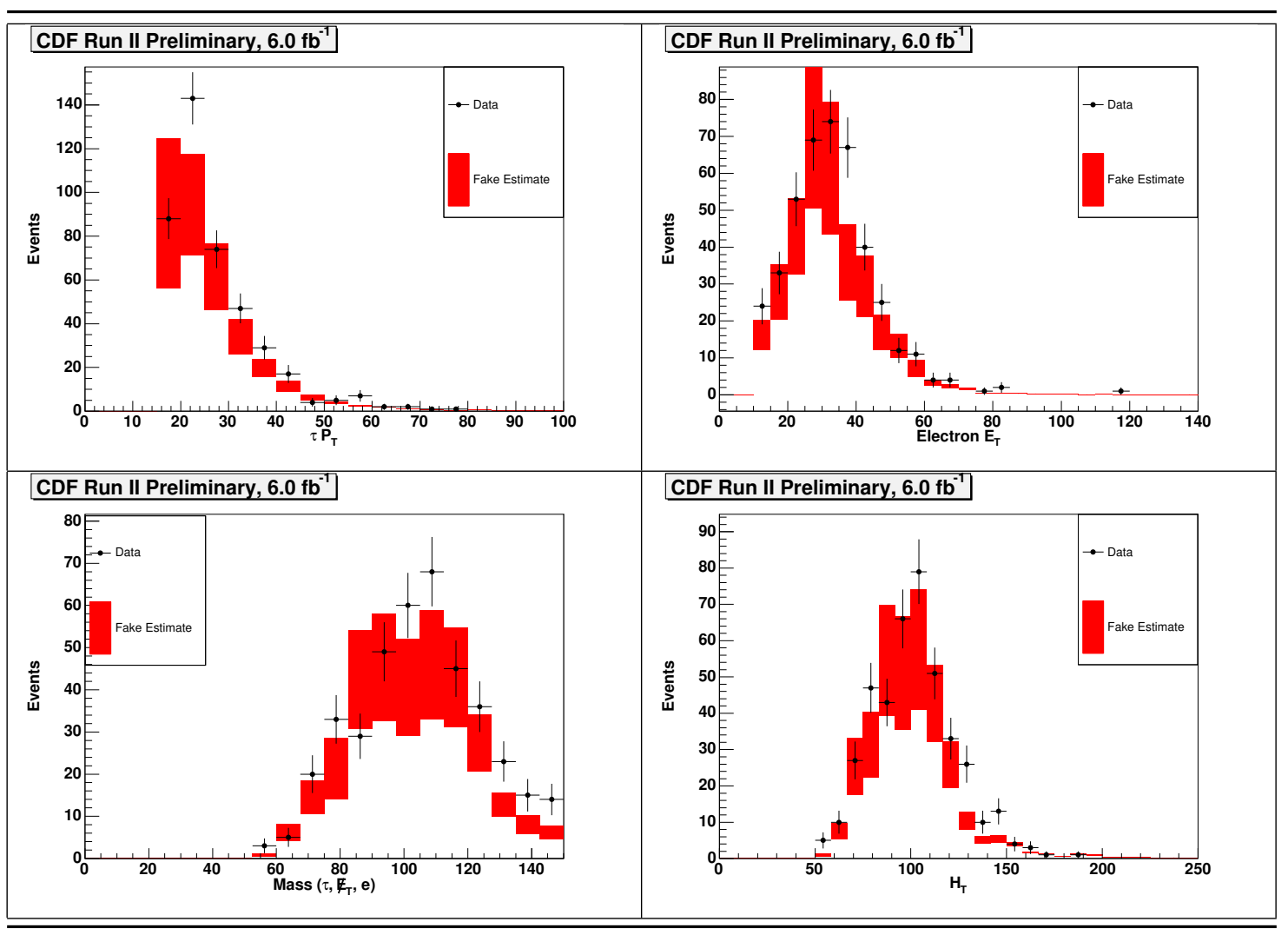

Figure 5.3: Fake Rate Verification. $W+$ Jet events with $W \rightarrow e \nu_{e}$. Bands formed by leading and sub-leading jets in fake rate measurement. Data points are from tight tau identification. 


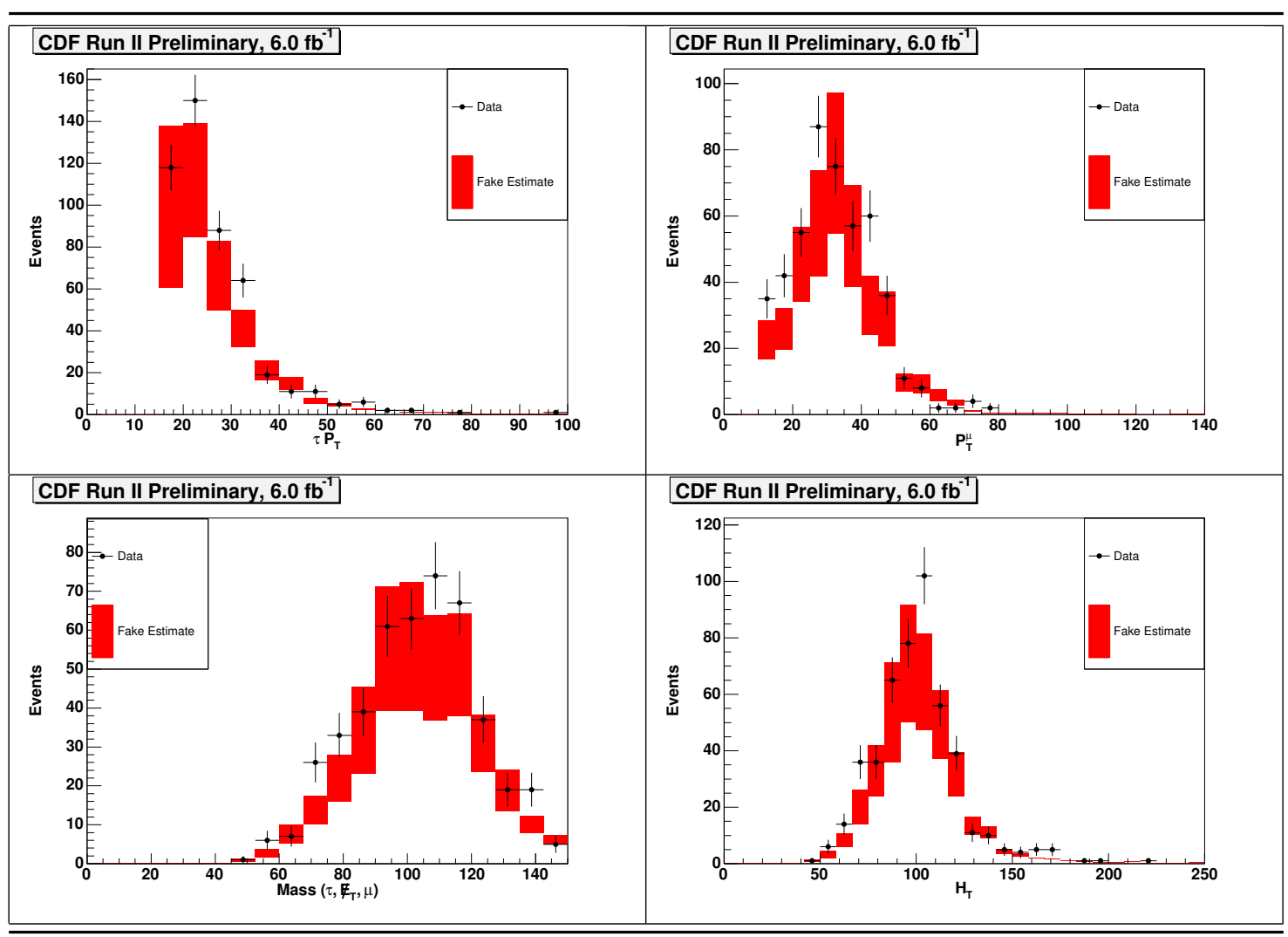

Figure 5.4: Fake Rate Verification. $W+$ Jet events with $W \rightarrow \mu \nu_{\mu}$. Bands formed by leading and sub-leading jets in fake rate measurement. Data points are from tight tau identification. 


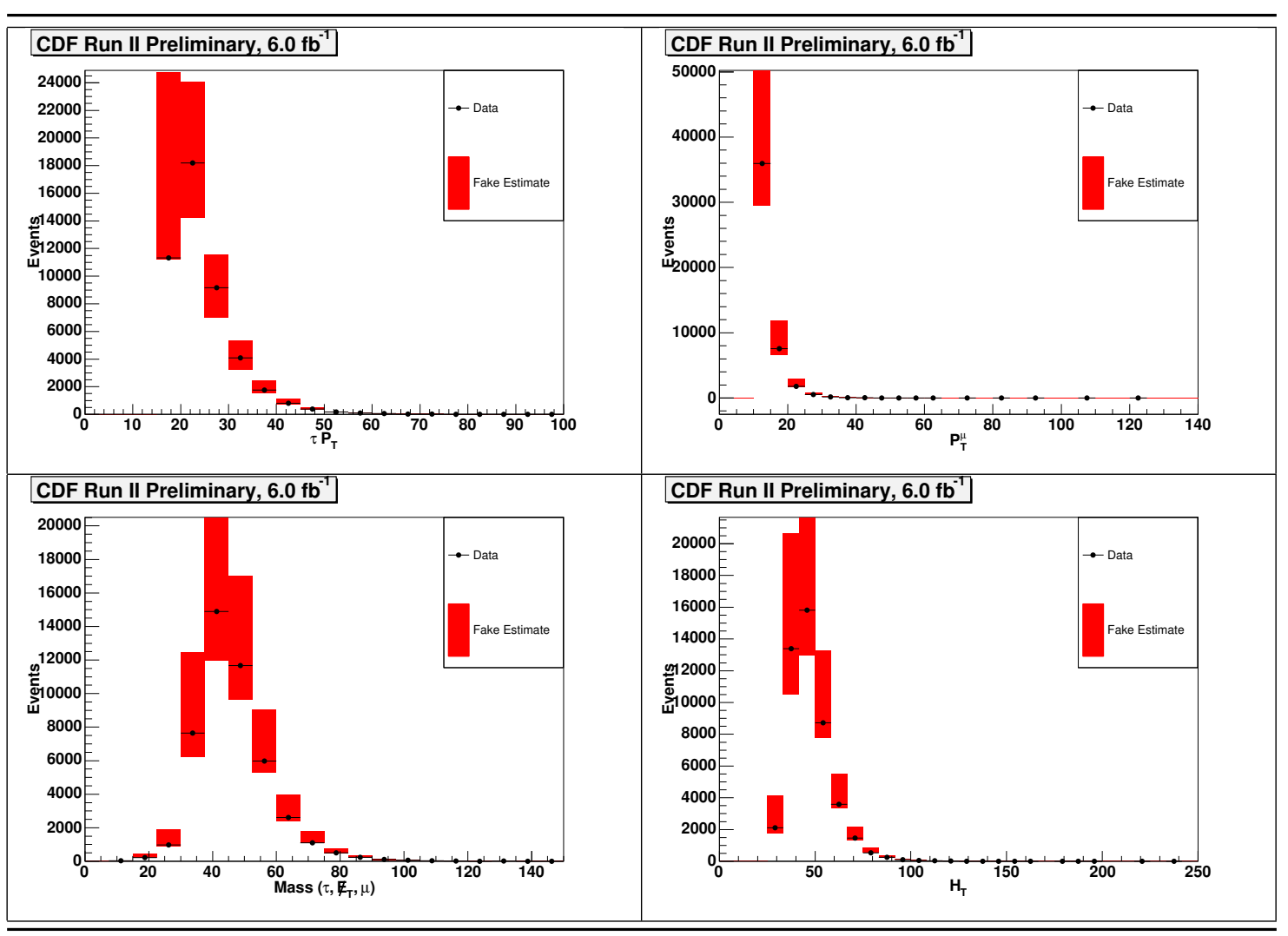

Figure 5.5: Fake Rate Verification. Non-isolated muons. Bands formed by leading and sub-leading jets in fake rate measurement. Data points are from tight tau identification. 


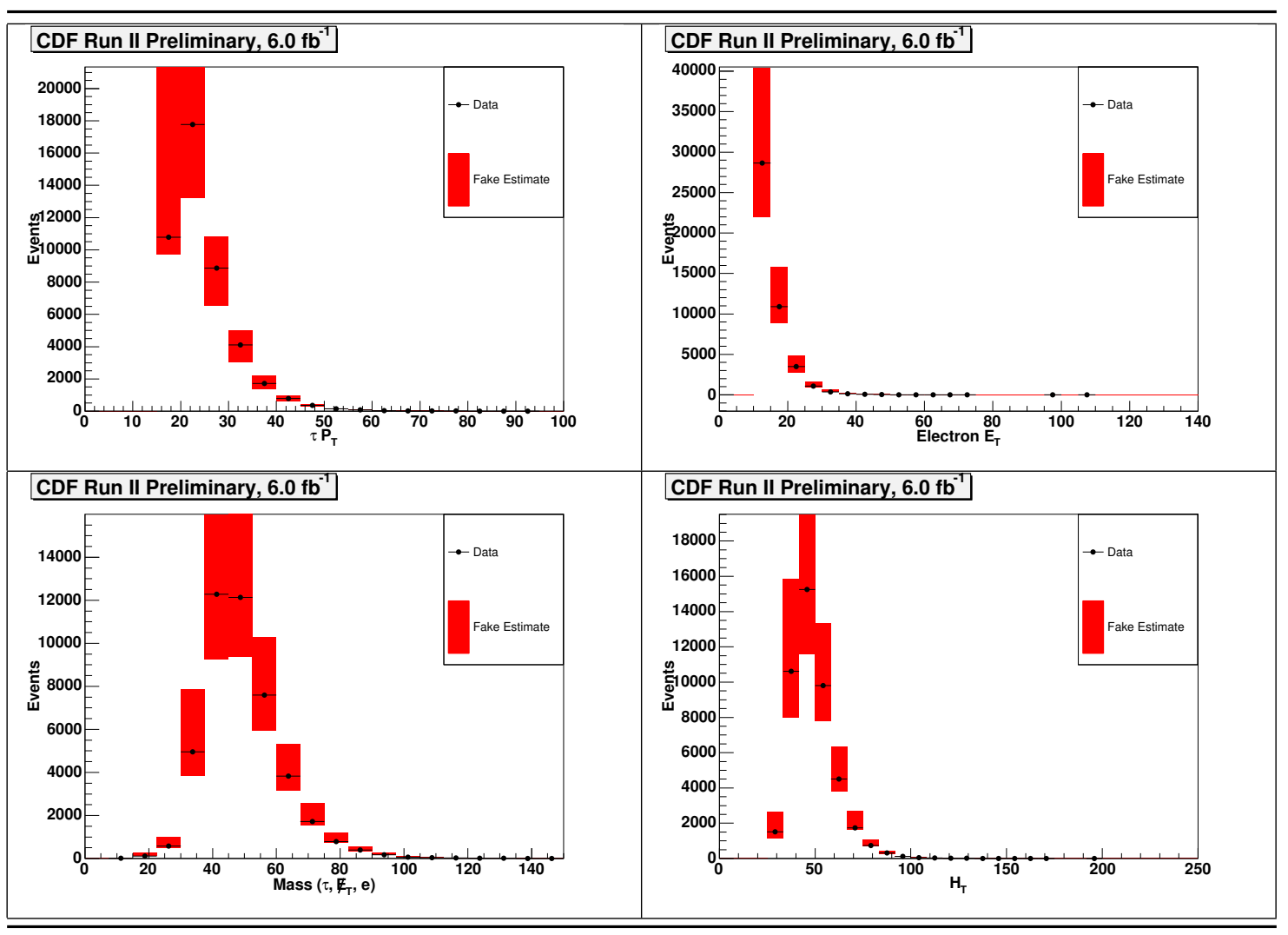

Figure 5.6: Fake Rate Verification. Non-isolated electrons. Bands formed by leading and sub-leading jets in fake rate measurement. Data points are from tight tau identification. 
taus. All event level cuts are also applied. We then weight them by the appropriately parameterized fake rate.

Applying the fake rate to the analysis dataset is complicated by the fact that the track side of the lepton + track trigger is very isolated, and therefore, by design, has a high efficiency of selecting real taus. Because the jet-to-tau fake rate method should account only for QCD jets faking taus, if real taus are a significant fraction of events, they will pass loose tau cuts, and cause an overestimation in the fake rate. Thus, when applying the fake rate, real tau contamination must be subtracted off. We follow the procedure here as outlined previously.

If $\hat{N}$ is the number of loose taus and $N$ is the number of tight taus, both real taus and jets contribute to candidates passing our tight and loose cuts.

$$
\begin{array}{r}
\hat{N}=\hat{N}^{\tau}+\hat{N}^{j e t} \\
N=N^{\tau}+N^{j e t} \\
N^{\tau}=\epsilon \hat{N}^{\tau} \\
N^{j e t}=f \hat{N}^{j e t}
\end{array}
$$

Where (5.3) is the expression for the efficiency of finding a real tau and (5.4) is the expression for the fake rate. We can rewrite the expression for $N^{j e t}$ as:

$$
N^{j e t}=\frac{f}{\epsilon-f}(\epsilon \hat{N}-N)
$$

We parameterize the fake fate with some arbitrary variables $(\Omega)$ such as cone $E_{T}$ and detector $\eta$, to take this into account we write the above equation for small regions in parameter space to get the expression in terms of the fake tau density $\hat{n}$ and $n$ as a function of $\Omega$, instead of the numbers of events $\hat{N}$ and $N$ : 


$$
\begin{array}{r}
n^{j e t}(\Omega)=\frac{f(\Omega)}{\epsilon(\Omega)-f(\Omega)}[\epsilon(\Omega) \hat{n}(\Omega)-n(\Omega)] \\
\hat{n}(\Omega)=\sum_{i}^{\hat{N}} \delta\left(\Omega-\Omega_{i}\right) \\
n(\Omega)=\sum_{i}^{N} \delta\left(\Omega-\Omega_{i}\right)
\end{array}
$$

We substitute the densities for $\hat{n}(\Omega)$ and $n(\Omega)$ into 5.6, integrate and extract the coefficients of $\hat{n}(\Omega)$ and $n(\Omega)$. Note that we define $\hat{N}$ as loose taus that are not tight, so even though $N$ is a subset of $\hat{N}$, they contribute only via 5.8 below, and $\hat{N}+N$ is the total number of taus that contribute to this estimate. The results for tight and loose taus are:

$$
\begin{array}{r}
w_{i}^{\text {loose }}=\frac{f\left(\Omega_{i}\right) \epsilon\left(\Omega_{i}\right)}{\epsilon\left(\Omega_{i}\right)-f\left(\Omega_{i}\right)} \\
w_{i}^{\text {tight }}=\frac{f\left(\Omega_{i}\right) \epsilon\left(\Omega_{i}\right)-1}{\epsilon\left(\Omega_{i}\right)-f\left(\Omega_{i}\right)}
\end{array}
$$

Notice the efficiency will generally be larger than fake rate, therefore the contribution from tight taus is negative. This confirms the fact that we are subtracting from the fake rate estimate the contamination from real taus.

\subsubsection{Jet-to- $\tau$ Fake Rate Conversion Corrections}

We observe one verification region in Figure 5.2 in which the data and fake rate prediction are systematically discrepant. In the measurement of the fake rate for conversion rich events, there is a slight measurement bias by choosing the leading jet for our upper limit on the fake rate, since the highest $E_{t}$ jet in QCD events is most likely to be a quark jet. However, in the process $q \bar{q} \rightarrow \gamma+g$ we have dominantly gluon jets to first and second order. This effect can be seen only in the electron channel, with a jet faking a tau and a photon converting to an electron. So, although we have the correctly tagged events in our validation sample, we need to apply a correction to arrive at the appropriate normalization. To do this, we use a sideband of the SS 
data, $\mathbb{E}_{T}<20 \mathrm{GeV}$ that will not be used. We normalize all backgrounds to their measured cross sections, apply the fake rate as described in Section 5.2.3 and float a sample of tagged conversions to fit to data. We arrive at a contribution of $289.6 \pm 10.7$ conversion events that will be added to both the OS and SS sample.

We then crosscheck this extra conversion contribution with a different method. We measure a scale factor in the OS subset of Figure 5.2 and find it to be 1.55 between data and our central fake prediction. This scale factor needs to be applied to the fraction of events in our final sample that are conversions. To get the conversion composition of our events we measure the effect of the conversion veto on the analysis, which is 778 vetoed events. Combining this with the knowledge that the efficiency of the conversion cut is $60 \%$, we calculate that we need to add 285 additional conversion events to the final background. This agrees well with the $289.6 \pm 10.7$ we calculated by fitting to data.

Because of the shape of this particular background, very few events will make it into our final signal sample, for a $\mathbb{E}_{T}$ cut of $20 \mathrm{GeV}$ it comprises less than $4 \%$ of the total background in the electron channel.

A systematic error on the conversion correction is applied. The difference in scale factors between the OS and SS subsets of Figure 5.2 is $25 \%$ and the error on the conversion cut efficiency is about $5 \%$. We then use a generous systematic error of $30 \%$ on the conversion correction background. 


\section{Chapter 6}

\section{Opposite-Signed Control Region}

Our primary control region is the opposite-signed region. Unlike our signal region, we expect this to be dominated by $Z \rightarrow \tau \tau$ events with $W+$ Jet events the secondary background. This control region allows us to test both the scale factors, which do not affect the fakes; as well as the fake rate method. Systematic errors are discussed in the following chapter.

\section{$6.1 \quad H_{T}$ Cut}

All standard event level cuts have been mentioned in this note except the $H_{T}$ cut, which serves to reduce the QCD background and is applied in both OS and SS regions. We define $H_{t}$ as:

$$
H_{t}=\left|P_{t}^{\tau}\right|+\left|P_{t}^{l}\right|+\mathbb{E}_{T}
$$

We vary the value of this cut based on the expected contamination from misidentified jets. Three-pronged jets provide a large fake contamination, so we place our highest $H_{T}$ cut of $55 \mathrm{GeV}$ on all 3-pronged tau events. For one-pronged taus, the electron has a slightly higher fake rate than a muon, so our cuts are lowered to 50 $\mathrm{GeV}$ for the former, and $45 \mathrm{GeV}$ for the latter. 


\subsection{Opposite-Signed Results}

The event counts are given in Tables 6.1, 6.2, and 6.3 for the opposite-signed control regions.

\begin{tabular}{|c|c|}
\hline \multicolumn{2}{|c|}{$\begin{array}{l}\text { CDF Run II Preliminary } 6.0 \mathrm{fb}^{-1} \\
\text { OS } e-\tau\end{array}$} \\
\hline Process & Events \pm stat \pm syst \\
\hline $\mathrm{Z} \rightarrow \tau \tau$ & $3258.5 \pm 38.5 \pm 260.7$ \\
\hline $\mathrm{Jet} \rightarrow \tau$ & $2570.2 \pm 21.1 \pm 577.6$ \\
\hline $\mathrm{Z} \rightarrow \mu \mu$ & $0.5 \pm 0.9 \pm 0.0$ \\
\hline $\mathrm{Z} \rightarrow e e$ & $82.5 \pm 8.6 \pm 6.6$ \\
\hline $\mathrm{W} \rightarrow \tau \nu$ & $182.0 \pm 8.7 \pm 17.8$ \\
\hline$t \bar{t}$ & $17.8 \pm 0.2 \pm 2.5$ \\
\hline Diboson & $30.3 \pm 0.7 \pm 3.0$ \\
\hline Total & $6141.9 \pm 45.6 \pm 634.0$ \\
\hline Data & 6058 \\
\hline
\end{tabular}

Table 6.1: OS electron channel control region.

Below are some representative plots followed by additional plots with backgrounds broken down by component. The agreement of the data with background gives us confidence in our background model. 


\begin{tabular}{l|r|}
\hline \multicolumn{2}{|l|}{ CDF Run II Preliminary $6.0 \mathrm{fb}^{-1}$} \\
OS $\mu-\tau$ \\
\hline \hline Process & Events \pm stat \pm syst \\
\hline $\mathrm{Z} \rightarrow \tau \tau$ & $3708.8 \pm 41.1 \pm 296.7$ \\
$\mathrm{Jet} \rightarrow \tau$ & $1956.2 \pm 16.5 \pm 489.8$ \\
$\mathrm{Z} \rightarrow \mu \mu$ & $262.0 \pm 20.1 \pm 21.0$ \\
$\mathrm{Z} \rightarrow e e$ & $0.0 \pm 0.0 \pm 0.0$ \\
$\mathrm{~W} \rightarrow \tau \nu$ & $189.5 \pm 8.9 \pm 18.6$ \\
$\mathrm{t} \overline{\mathrm{t}}$ & $18.5 \pm 0.2 \pm 2.6$ \\
Diboson & $31.0 \pm 0.7 \pm 3.0$ \\
\hline Total & $6166.1 \pm 49.4 \pm 573.4$ \\
Data & 6210 \\
\hline \hline
\end{tabular}

Table 6.2: OS muon channel control region.

\begin{tabular}{|c|c|}
\hline \multicolumn{2}{|c|}{$\begin{array}{l}\text { CDF Run II Preliminary } 6.0 \mathrm{fb}^{-1} \\
\text { OS } \ell-\tau\end{array}$} \\
\hline Process & Events \pm stat \pm syst \\
\hline $\mathrm{Z} \rightarrow \tau \tau$ & $6967.3 \pm 56.4 \pm 557.4$ \\
\hline Jet $\rightarrow \tau$ & $4526.5 \pm 26.8 \pm 1064.5$ \\
\hline $\mathrm{Z} \rightarrow \mu \mu$ & $262.5 \pm 20.1 \pm 21.0$ \\
\hline $\mathrm{Z} \rightarrow e e$ & $82.5 \pm 8.6 \pm 6.6$ \\
\hline $\mathrm{W} \rightarrow \tau \nu$ & $371.5 \pm 12.4 \pm 36.4$ \\
\hline $\mathrm{t} \overline{\mathrm{t}}$ & $36.3 \pm 0.3 \pm 5.1$ \\
\hline Diboson & $61.3 \pm 0.9 \pm 6.0$ \\
\hline Total & $12308.0 \pm 67.3 \pm 1202.3$ \\
\hline Data & 12268 \\
\hline
\end{tabular}

Table 6.3: Total OS control region. 


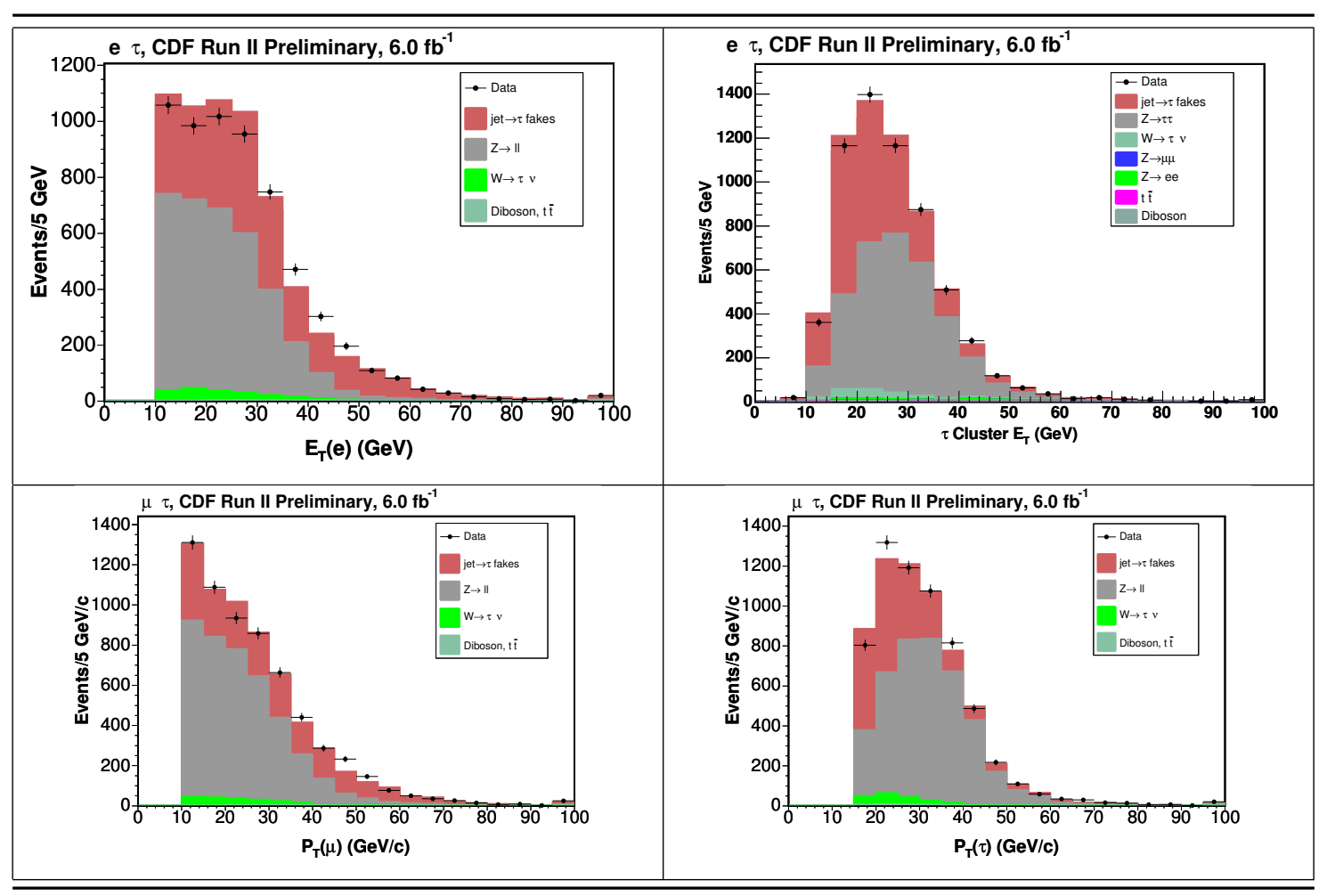

Figure 6.1: OS Control region plots. Electron channel (top) : Electron $E_{t}$ (left) and tau cluster $E_{T}$. Muon channel (bottom): Muon $P_{t}$ (left) and tau $P_{t}$ (right).

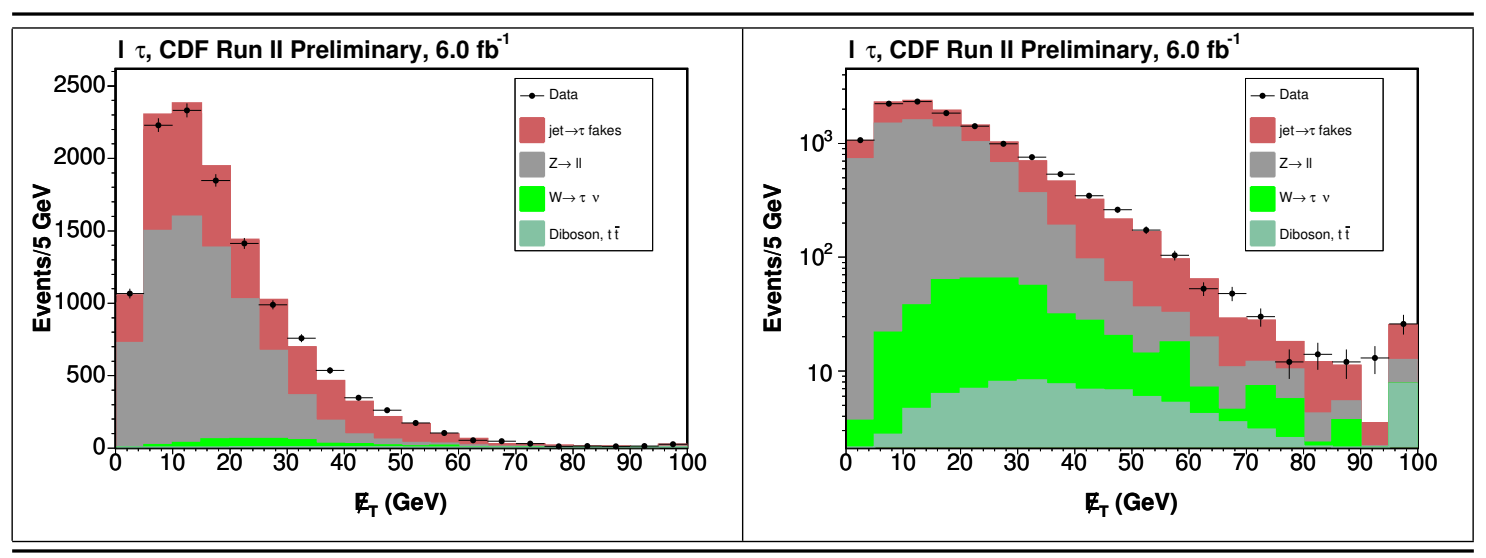

Figure 6.2: OS Control region plots. $\mathbb{E}_{T}$ linear scale (left) and log scale (right). 


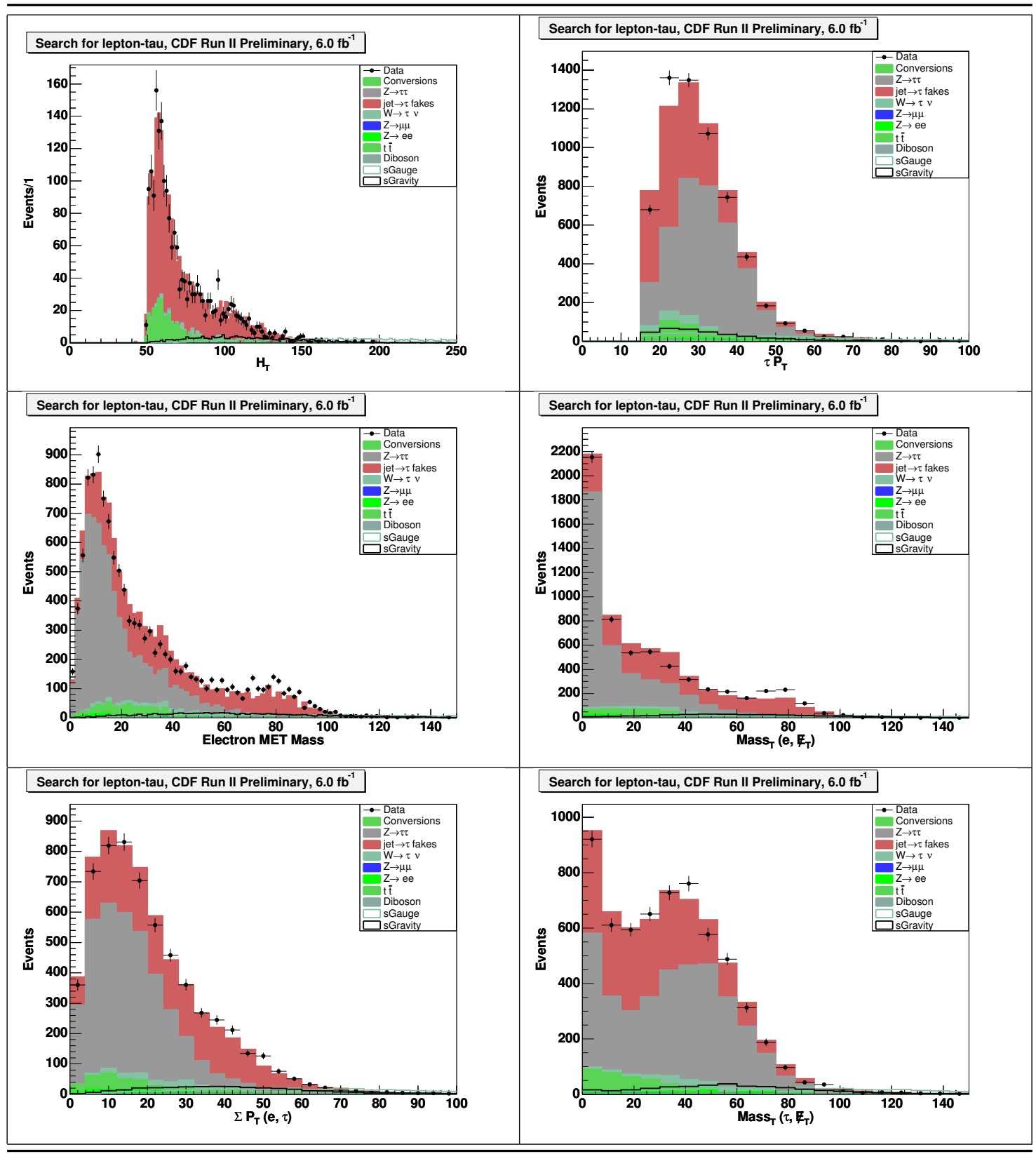

Figure 6.3: OS Control region plots. Electron channel: Electron $H_{t}$ (top left) and tau $P_{T}$ (top right). Mass of e and $\mathbb{E}_{T}$ (middle left), transverse mass of e and $E_{T}$ (middle right). Sum $P_{T}$ of the e and tau, (bottom left) and the transverse mass of the tau and the $\mathbb{E}_{T}$. 


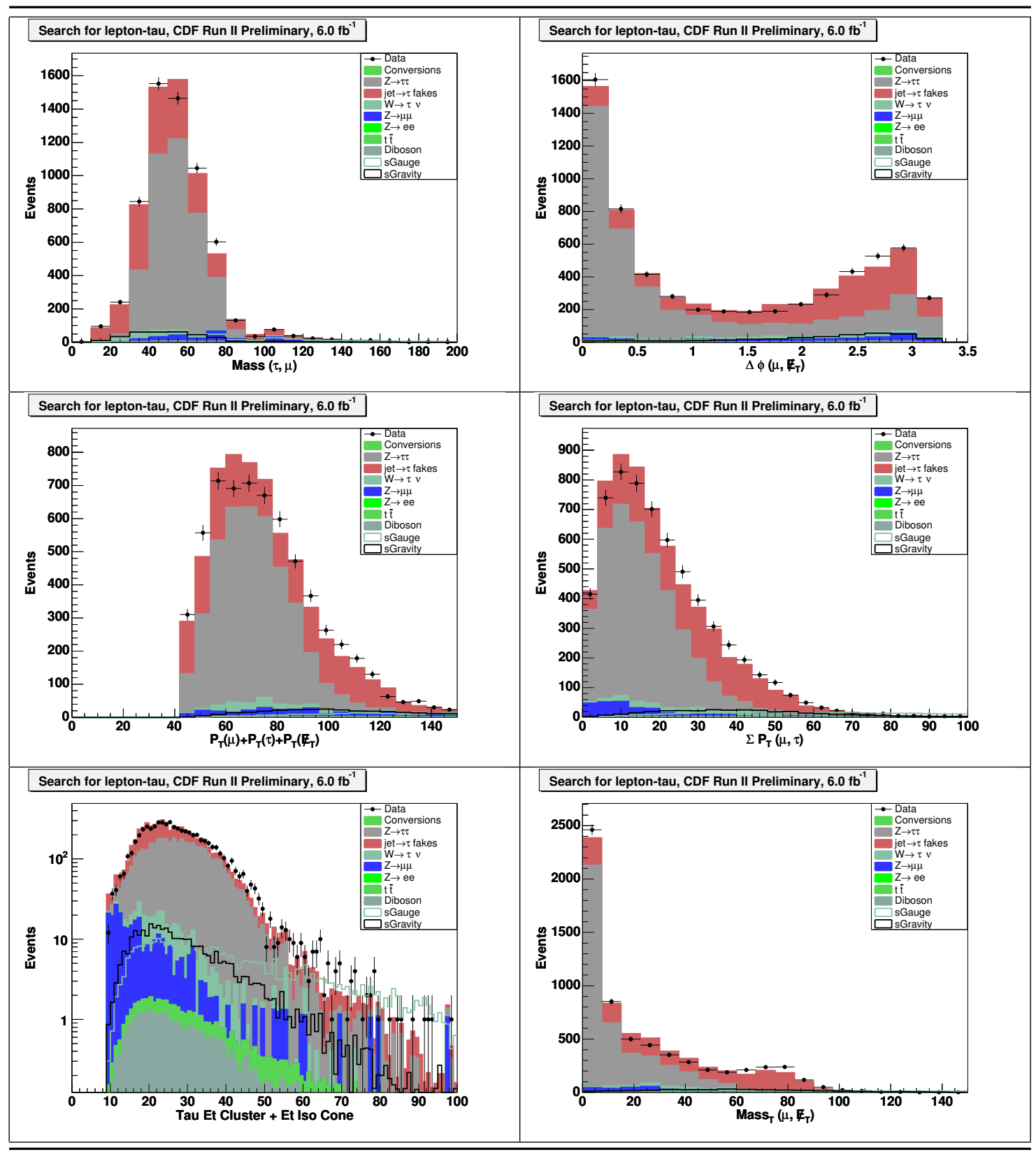

Figure 6.4: OS Control region plots. Muon channel: Muon, tau invariant mass (top left) and $d \phi\left(\mu, \mathbb{E}_{T}\right)$ (top right). Sum $P_{t}\left(\mu, \tau, \mathbb{E}_{T}\right)$ (middle left), Sum $P_{t}(\mu, \tau)$ (middle right). $E_{T}$ of the tau in both the siganl and iso cone, (bottom left) and the transverse mass of the muon and the $\mathbb{E}_{T}$. 


\section{Chapter 7}

\section{Systematic Uncertainties}

The total uncertainty on the analysis results will be a combination of statistical and systematic uncertainties. Many systematic uncertainties have been mentioned throughout the text, we summarize a full list here. Generally, this will be a counting analysis, so we only have rate uncertainties and do not have shape fits that effect our final result. The systematic uncertainties comprise the following categories: trigger efficiency, identification and reconstruction efficiency, event cuts, background estimation, parton distribution functions (PDF) and luminosity. Generally, because of the composition of our background, the uncertainty on the tau fake rate will dominate. See Table 7.1 for a summary of all systematics used.

\subsection{Trigger Uncertainty}

The trigger efficiency measurement is covered in depth in section 4.2. Generally, we assign an uncertainty to each lepton and tau separately in MC, so each event has contributions from the lepton side and the tau side of the lepton + track trigger. For the tau side, we use a 3.0\% uncertainty. The electron trigger efficiency is calculated and the associated uncertainty is $0.3 \%$. The same is true for the two muons types, the CMUP trigger has a luminosity-weighted $0.7 \%$ uncertainty and the CMX trigger has the same, so all muon events have a $0.7 \%$ systematic. As mentioned in 4.2 , trigger efficiency measurements have been shown to be stable below the $20 \mathrm{GeV} / c$ threshold of the standard CDF inclusive lepton triggers. 


\subsection{Reconstruction and ID Uncertainty}

We need to measure the uncertainty on the scale factors used in the analysis to correct for differences between data and simulated MC event reconstruction in the detector. Here we examine uncertainties both leptons and taus spanning their whole $P_{T}$ range.

As mentioned in section 4.3, for the MC modeled processes in the background, we use standard CDF selection for high $P_{T}(>20 \mathrm{GeV} / c)$ leptons up to the isolation requirement. There are different selection requirements used for leptons below this threshold. We weight the systematic by the fraction of the leptons in the final sample in each category.

Including isolation, a $1 \%$ reconstruction uncertainty for muons over $P_{T}>20$ $\mathrm{GeV} / c$ covers all muon types. For low $P_{T}$ muons we use a $3.4 \%$ systematic that covers both the muon types. We take the fraction of muons above and below the 20 $\mathrm{GeV} / c$ threshold and assign a $2.0 \%$ systematic for muon ID and Reconstruction.

Electrons are similarly divided into categories above and below $E_{t}=20 \mathrm{GeV}$. For high $E_{t}$ electrons we use the common CDF $0.6 \%$ systematic uncertainty and we use the measurements of low $E_{T}$ electrons for a $1.2 \%$ uncertainty. Again weighing the final uncertainty by the final sample's high (65\%) and low (35\%) fraction, we assign a $0.7 \%$ uncertainty on electron ID and reconstruction.

For hadronically decaying taus, the total uncertainty is measured taking into account all object selection factors at once. It is found to be $3.0 \%$, which is applied to all taus in MC. This includes all effects mentioned in Section 4.3.3.2.

\section{$7.3 \quad Z_{0}$ Cut}

We apply a systematic uncertainty on the event-level cut requiring the primary vertex to be $\left|Z_{0}\right|<60 \mathrm{~cm}$. This is measured by CDF to have a $0.1 \%$ systematic uncertainty.

\subsection{Standard Model Cross Sections}

There is a systematic uncertainty on all standard model cross sections. We normalize the MC signal to its known cross section, so the uncertainty on the known cross 
section corresponds to the systematic uncertainty on each respective MC process in the analysis. The standard uncertainties used at CDF are documented in many analysis. The uncertainty is $2.2 \%$ for $\mathrm{Z} \rightarrow e e, \mu \mu$ [43], $10.0 \%$ for $\mathrm{t} \overline{\mathrm{t}}$ production [44] and $6.0 \%$ for the diboson processes [45].

\subsection{Conversions}

The uncertainty on the conversion finding efficiency is $30 \%$ as measured by the deviation between two measurement techniques as described in Section 5.2.4.

\subsection{Luminosity}

CDF has a standard luminosity measurement systematic uncertainty of $6.0 \%$, applied only to the MC backgrounds [46].

\subsection{Jet $\rightarrow \tau$ Fake Rate}

By far the dominant systematic uncertainty of the analysis is the Jet $\rightarrow \tau$ fake rate as described in Section 5.2. It is measured to be $25 \%$ and is applied to all jet fake backgrounds.

\subsection{Jet Energy Scale}

Following the standard CDF prescription, we alter our jet energy corrections by $\pm 1 \sigma$ and note the change in acceptance in our sample. Our systematic uncertainty from jet energy scale is $1.5 \%$ and is applicable to all backgrounds [47].

\subsection{Tau Energy Scale}

The tau energy is shifted as outlined in Section 4.3.3.2, we take a $1 \%$ systematic uncertainty due to this shifting and apply it to all MC taus [39]. 


\begin{tabular}{llr}
\hline \hline Parameter & Applied To & Value $(\%)$ \\
\hline \hline Tau Side Trigger & Taus in MC & 3.0 \\
Electron Side Trigger & Electrons in MC & 0.3 \\
Muon Side Trigger & Muons in MC & 0.7 \\
\hline Muon ID/Reco & Muons in MC & 2.0 \\
Electron ID/Reco & Electrons in MC & 0.7 \\
Tau ID/Reco & Taus in MC & 3.0 \\
\hline$Z_{0} \mid<60$ cm Cut & All MC & 0.5 \\
\hline$\sigma(Z \rightarrow l$ l) & Z MC & 2.2 \\
$\sigma(\mathrm{t} \overline{\mathrm{t}})$ & $\mathrm{t} \overline{\mathrm{t}} \mathrm{MC}$ & 10.0 \\
$\sigma($ diboson $)$ & diboson MC & 6.0 \\
$\sigma($ W $)$ & W MC & 6.0 \\
Conversions $\epsilon$ & Conversions & 30.0 \\
\hline Luminosity & All MC & 6.0 \\
\hline Jet $\rightarrow \tau$ Fake Rate & Fake Rate Background & 25.0 \\
\hline Jet Energy Scale & All Backgrounds & 1.5 \\
\hline Tau Energy Scale & All MC & 1.0 \\
\hline \hline
\end{tabular}

Table 7.1: A summary of all systematic uncertainties used in the analysis, what they are applied to, and their value (\%). 


\section{Chapter 8}

\section{Results}

After establishing confidence in our fake rate measurement, as well as our background model, we unblind the data in the signal region. We now present the results for the same signed signal region. Since we see no excess in data over the expected background, we set limits on the cross section of our two generic signal models.

In this chapter I present the observed data in the same signed signal regions, including tables and plots of various distributions. I then look at certain low background sub-regions of data that were defined a priori, and the data that lies in those regions. I then set limits on the simplified models by finding optimized $\mathbb{E}_{T}$ cuts at each point in exclusion space. Extrapolated SUSY cross section contours are presented for each of the models and represent the final product of this analysis.

\subsection{Same Signed Data Results}

After unblinding the signal region, we look at the same signed signal region. Our ultimate limits are set with data above $\mathbb{E}_{T}=20 \mathrm{GeV}$. The result for that region is shown in Tables 8.1, 8.2 and 8.3.

We now show plots of various distributions for the SS signal region. The kinematical plots show there is good agreement between the background prediction and the data. We show several versions of the vital plots, for example the $\mathbb{E}_{T}$ plots are shown 


\begin{tabular}{l|r|}
\hline \multicolumn{2}{|l|}{ CDF Run II Preliminary $6.0 \mathrm{fb}^{-1}$} \\
$\mathrm{SS} \ell-\tau$ \\
\hline \hline Process & Events \pm stat. \pm syst. \\
\hline $\mathrm{Z} \rightarrow \tau \tau$ & $10.2 \pm 2.2 \pm 0.8$ \\
$\mathrm{Jet} \rightarrow \tau$ & $1152.7 \pm 15.2 \pm 283.1$ \\
$\mathrm{Z} \rightarrow \mu \mu$ & $0.0 \pm 0.0 \pm 0.0$ \\
$\mathrm{Z} \rightarrow e e$ & $0.0 \pm 0.0 \pm 0.0$ \\
$\mathrm{~W} \rightarrow \tau \nu$ & $96.9 \pm 6.4 \pm 9.5$ \\
$\mathrm{t} \overline{\mathrm{t}}$ & $0.7 \pm 0.0 \pm 0.1$ \\
Diboson & $4.3 \pm 0.2 \pm 0.4$ \\
\hline Total & $1264.8 \pm 16.6 \pm 283.3$ \\
Data & 1116 \\
\hline \hline
\end{tabular}

Table 8.1: SS signal region used in limit setting, $\mathbb{E}_{T}>20 \mathrm{GeV}$. Both e and $\mu$ channels.

\begin{tabular}{l|r|}
\hline \multicolumn{2}{|l|}{ CDF Run II Preliminary $6.0 \mathrm{fb}^{-1}$} \\
$\mathrm{SS} e-\tau$ \\
\hline \hline Process & Events \pm stat. \pm syst. \\
\hline $\mathrm{Z} \rightarrow \tau \tau$ & $5.0 \pm 1.5 \pm 0.4$ \\
$\mathrm{Jet} \rightarrow \tau$ & $537.0 \pm 10.4 \pm 129.0$ \\
$\mathrm{Z} \rightarrow \mu \mu$ & $0.0 \pm 0.0 \pm 0.0$ \\
$\mathrm{Z} \rightarrow e e$ & $0.0 \pm 0.0 \pm 0.0$ \\
$\mathrm{~W} \rightarrow \tau \nu$ & $43.2 \pm 4.2 \pm 4.2$ \\
$\mathrm{t} \overline{\mathrm{t}}$ & $0.4 \pm 0.0 \pm 0.0$ \\
Diboson & $2.1 \pm 0.2 \pm 0.2$ \\
\hline Total & $587.7 \pm 11.3 \pm 129.1$ \\
Data & 518 \\
\hline \hline
\end{tabular}

Table 8.2: SS signal region used in limit setting, $\mathbb{E}_{T}>20 \mathrm{GeV}$. Electron Channel.

in several formats since these are used in limit setting. 


\begin{tabular}{l|r|}
\hline \multicolumn{2}{|l|}{ CDF Run II Preliminary $6.0 \mathrm{fb}^{-1}$} \\
$\mathrm{SS} \mu-\tau$ \\
\hline \hline Process & Events \pm stat. \pm syst. \\
\hline $\mathrm{Z} \rightarrow \tau \tau$ & $5.1 \pm 1.5 \pm 0.4$ \\
$\mathrm{Jet} \rightarrow \tau$ & $615.7 \pm 11.2 \pm 154.2$ \\
$\mathrm{Z} \rightarrow \mu \mu$ & $0.0 \pm 0.0 \pm 0.0$ \\
$\mathrm{Z} \rightarrow e e$ & $0.0 \pm 0.0 \pm 0.0$ \\
$\mathrm{~W} \rightarrow \tau \nu$ & $53.7 \pm 4.7 \pm 5.3$ \\
$\mathrm{t} \overline{\mathrm{t}}$ & $0.4 \pm 0.0 \pm 0.0$ \\
Diboson & $2.3 \pm 0.2 \pm 0.2$ \\
\hline Total & $677.1 \pm 12.2 \pm 154.3$ \\
Data & 598 \\
\hline \hline
\end{tabular}

Table 8.3: SS signal region used in limit setting, $\mathbb{E}_{T}>20 \mathrm{GeV}$. Muon Channel. 


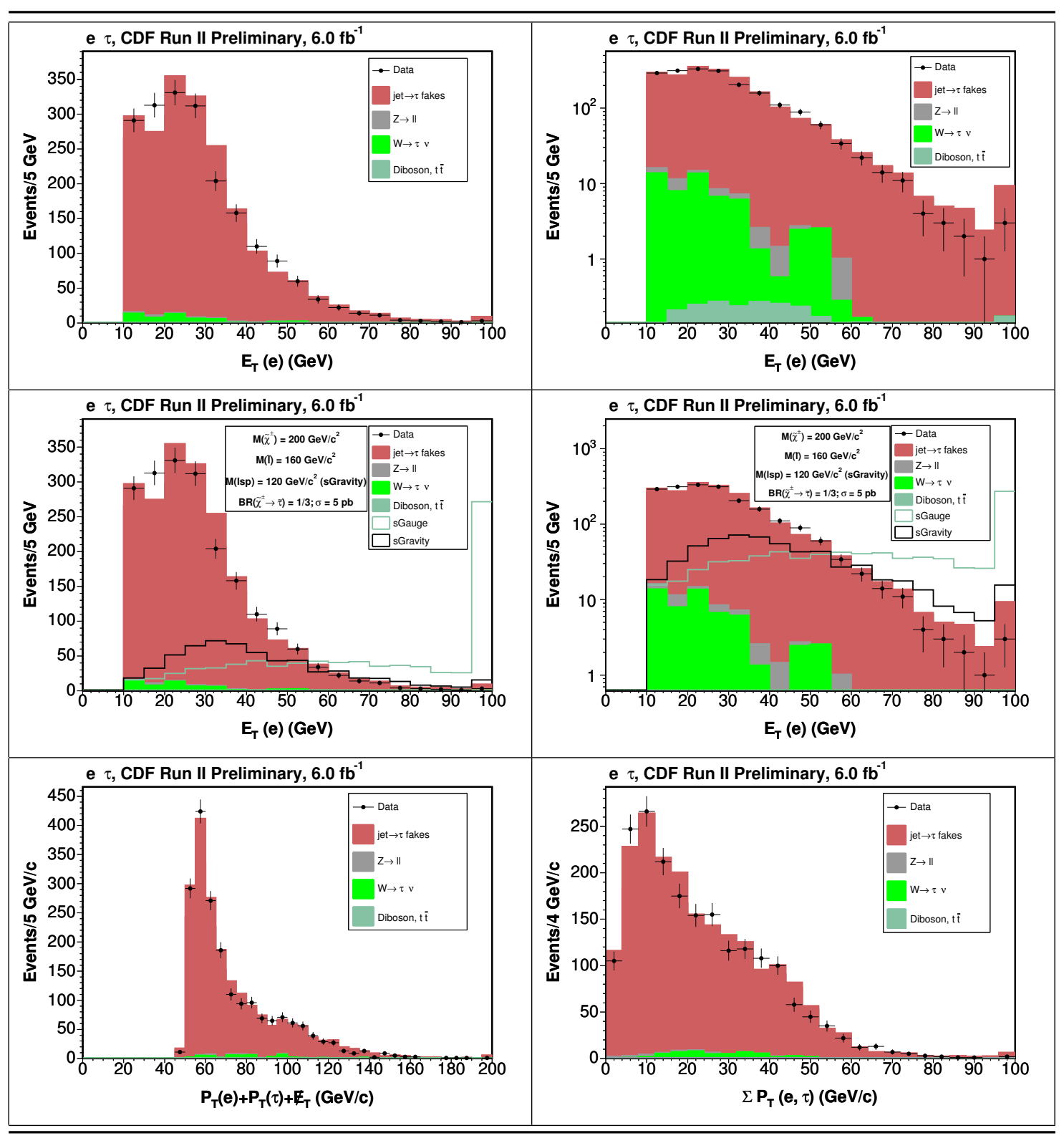

Figure 8.1: SS signal region plots, Electron channel: Electron $E_{t}$ (top left) and log version (top right). With signal points (middle left) and log versions (middle right). Electron $H_{T}$ (bottom left) and the $\Sigma P_{T}(e, \tau)$ (bottom right). 


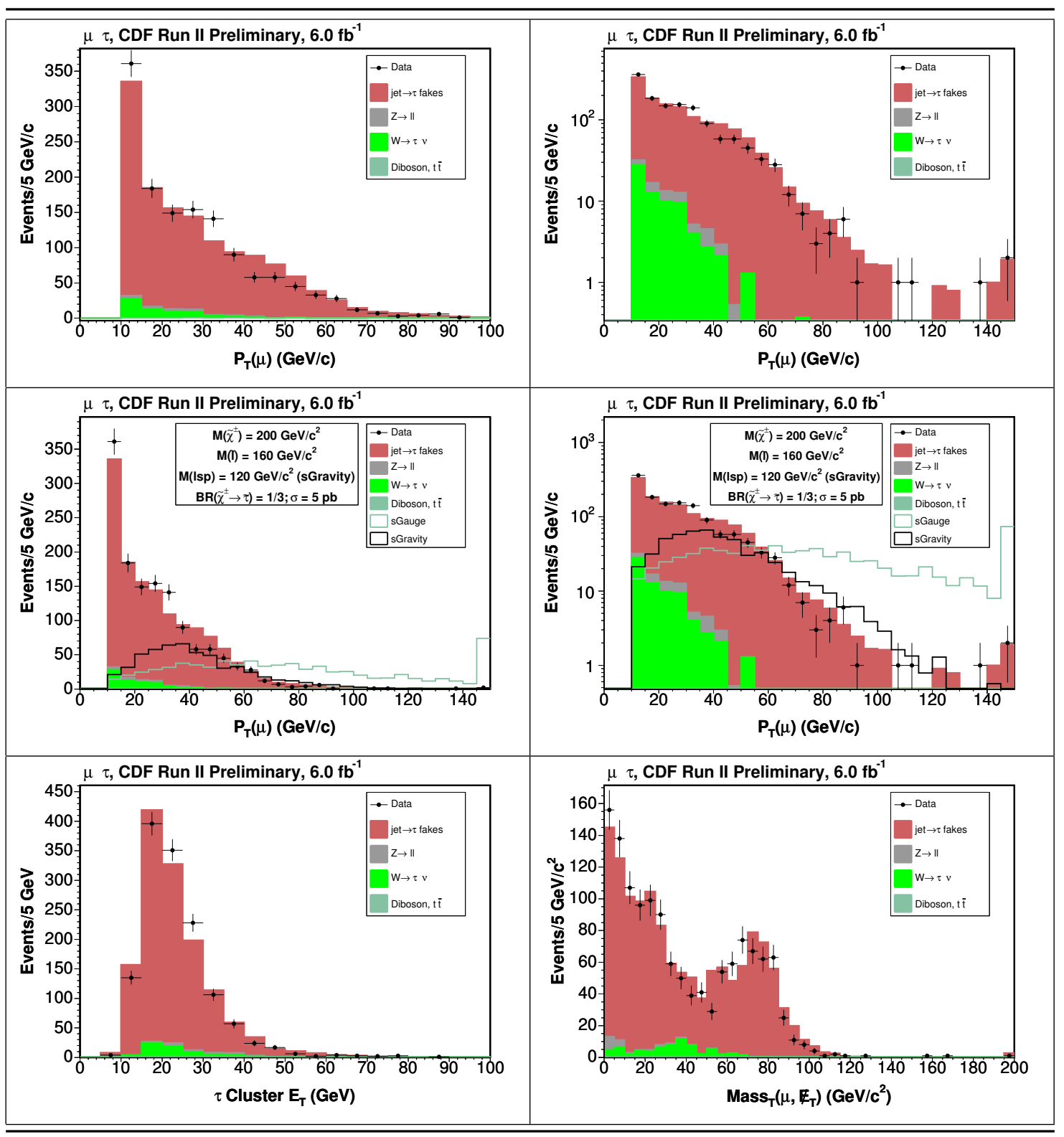

Figure 8.2: SS signal region plots, Muon channel: Muon $P_{t}$ (top left) and log version (top right). With signal points (middle left) and log versions (middle right). Tau Cluster $E_{T}$ (bottom left) and the $M_{T}\left(\mu, E_{T}\right)$ (bottom right). 


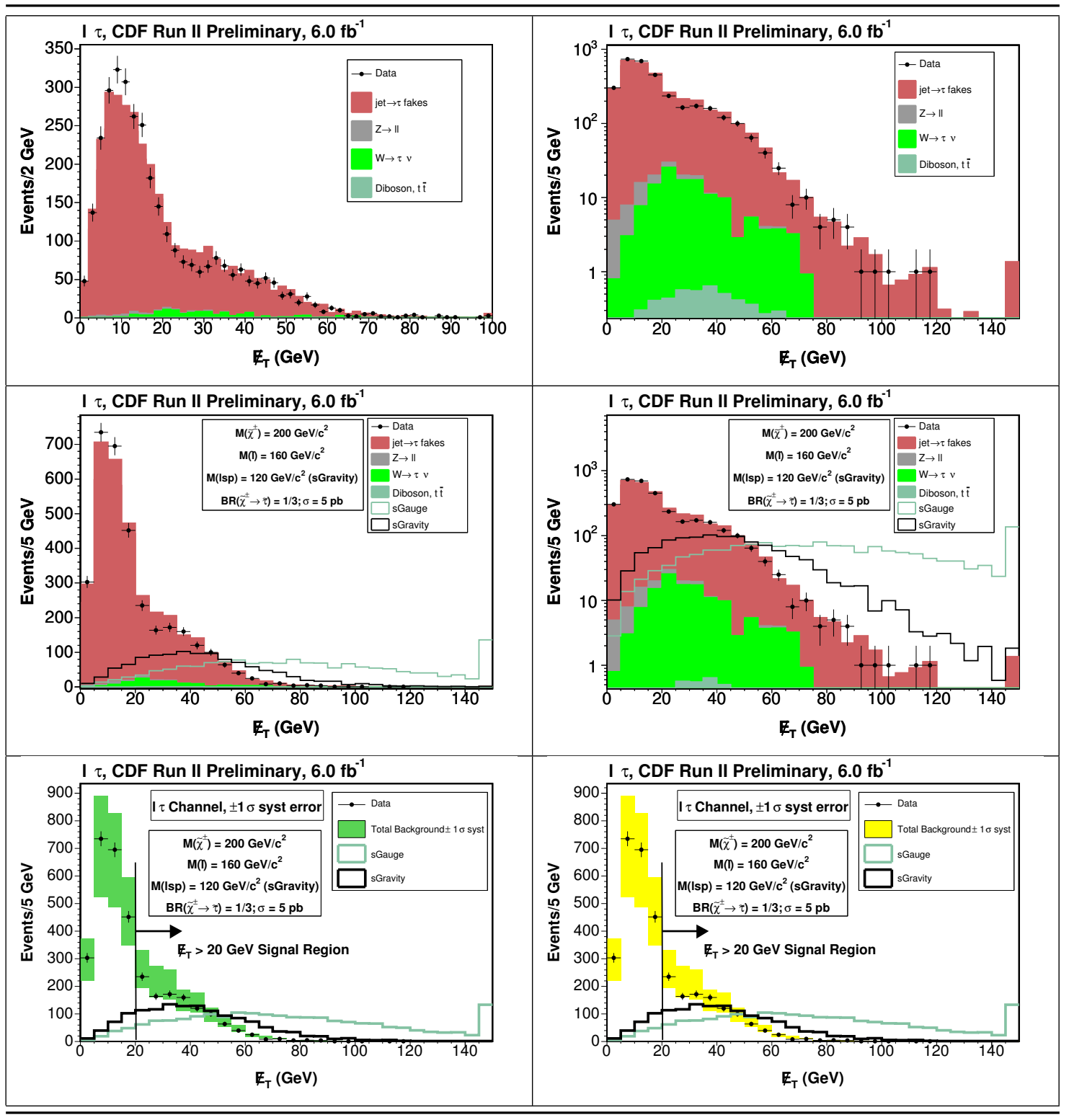

Figure 8.3: SS signal region plots, $\mathbb{E}_{T}$ plots for both channels: $\mathbb{E}_{T}$ (top left) and $\log$ version (top right). With signal points (middle left) and log versions (middle right). Total background with total systematic errors (bottom left) and (bottom right). 


\subsection{Reserved Signal Sub-Regions}

Since we are performing a blind analysis, it is prudent to determine, apriori, how to interpret any excess in particularly interesting low background sub-regions. To this end, we define three signal sub-regions and the expected backgrounds in each. We do this with no SUSY signal model, but merely to claim an excess if one does indeed appear in data. The regions are chosen in particularly high energy parameter space, away from standard model backgrounds. We observe no excess in these regions.

The regions are defined as follows:

- $M(\tau, 1)>200 \mathrm{GeV}$

- $H_{t}>300 \mathrm{GeV}$

- $P_{t}(\tau)>100 \mathrm{GeV} / c$ and $P_{t}(l)>100 \mathrm{GeV} / c$

Where $l$ represents either the electron or the muon in the event. The prediction and results for these sub-regions are shown below.

\subsection{1 $M(\tau, 1)>200 \mathrm{GeV} / c^{2}$}

The background prediction is show in Table 8.4.

\subsection{2 $H_{t}>300 \mathrm{GeV} / c$}

The background prediction is show in Table 8.5 and the distribution for this quantity is shown in Figure 8.4.

\subsection{3 $P_{t}(\tau)>100 \mathrm{GeV} / c$ and $P_{t}(l)>100 \mathrm{GeV} / c$}

The background prediction and result is show in Table 8.6. 


\begin{tabular}{|c|c|}
\hline \multicolumn{2}{|c|}{$\begin{array}{l}\text { CDF Run II Preliminary } 6.0 \mathrm{fb}^{-1} \\
\text { SS } \ell-\tau\end{array}$} \\
\hline Process & Events \pm stat \pm syst \\
\hline $\mathrm{Z} \rightarrow \tau \tau$ & $0.0 \pm 0.0 \pm 0.0$ \\
\hline Jet $\rightarrow \tau$ & $3.3 \pm 0.4 \pm 0.8$ \\
\hline $\mathrm{Z} \rightarrow \mu \mu$ & $0.0 \pm 0.0 \pm 0.0$ \\
\hline $\mathrm{Z} \rightarrow e e$ & $0.0 \pm 0.0 \pm 0.0$ \\
\hline $\mathrm{W} \rightarrow \tau \nu$ & $0.0 \pm 0.0 \pm 0.0$ \\
\hline $\mathrm{t} \overline{\mathrm{t}}$ & $0.0 \pm 0.0 \pm 0.0$ \\
\hline Diboson & $0.1 \pm 0.0 \pm 0.0$ \\
\hline Total & $3.4 \pm 0.4 \pm 0.8$ \\
\hline Data & 0 \\
\hline
\end{tabular}

Table 8.4: Prediction for Sub-region: $M(\tau, l)>200 \mathrm{GeV} / c^{2}$

\begin{tabular}{|c|c|}
\hline \multicolumn{2}{|c|}{$\begin{array}{l}\text { CDF Run II Preliminary } 6.0 \mathrm{fb}^{-1} \\
\text { SS } \ell-\tau\end{array}$} \\
\hline Process & Events \pm stat \pm syst \\
\hline $\mathrm{Z} \rightarrow \tau \tau$ & $0.0 \pm 0.0 \pm 0.0$ \\
\hline Jet $\rightarrow \tau$ & $2.1 \pm 0.4 \pm 0.5$ \\
\hline $\mathrm{Z} \rightarrow \mu \mu$ & $0.0 \pm 0.0 \pm 0.0$ \\
\hline $\mathrm{Z} \rightarrow e e$ & $0.0 \pm 0.0 \pm 0.0$ \\
\hline $\mathrm{W} \rightarrow \tau \nu$ & $0.0 \pm 0.0 \pm 0.0$ \\
\hline$t \bar{t}$ & $0.0 \pm 0.0 \pm 0.0$ \\
\hline Diboson & $0.1 \pm 0.0 \pm 0.0$ \\
\hline Total & $2.2 \pm 0.4 \pm 0.5$ \\
\hline Data & 1 \\
\hline
\end{tabular}

Table 8.5: Prediction for Sub-region: $H_{t}>300 \mathrm{GeV} / c$ 


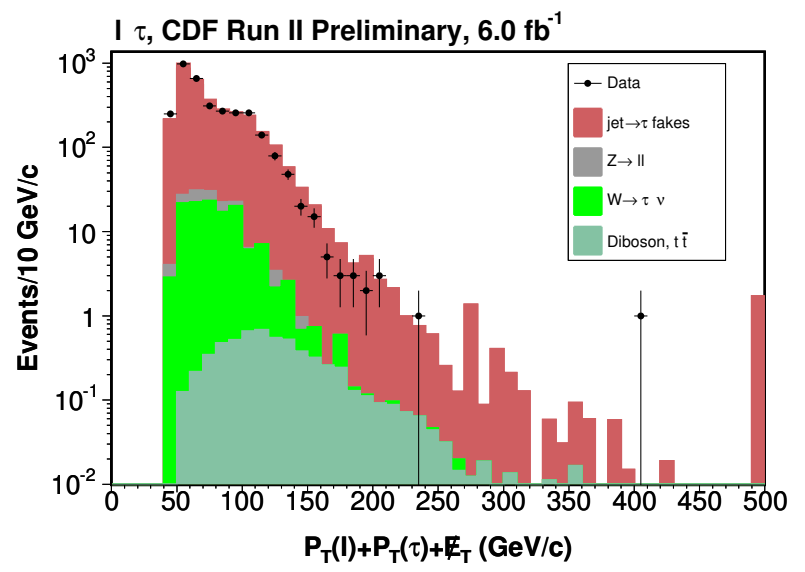

Figure 8.4: Distribution for Sub-region: $H_{t}$.

\begin{tabular}{|l|r|}
\hline \multicolumn{2}{|l|}{ CDF Run II Preliminary $6.0 \mathrm{fb}^{-1}$} \\
$\mathrm{SS} \ell-\tau$ \\
\hline \hline Process & Events \pm stat \pm syst \\
\hline $\mathrm{Z} \rightarrow \tau \tau$ & $0.0 \pm 0.0 \pm 0.0$ \\
$\mathrm{Jet} \rightarrow \tau$ & $0.7 \pm 0.1 \pm 0.2$ \\
$\mathrm{Z} \rightarrow \mu \mu$ & $0.0 \pm 0.0 \pm 0.0$ \\
$\mathrm{Z} \rightarrow e e$ & $0.0 \pm 0.0 \pm 0.0$ \\
$\mathrm{~W} \rightarrow \tau \nu$ & $0.0 \pm 0.0 \pm 0.0$ \\
$\mathrm{t} \overline{\mathrm{t}}$ & $0.0 \pm 0.0 \pm 0.0$ \\
Diboson & $0.0 \pm 0.0 \pm 0.0$ \\
\hline Total & $0.7 \pm 0.1 \pm 0.2$ \\
Data & 0 \\
\hline \hline
\end{tabular}

Table 8.6: Prediction for Sub-region: $P_{t}(\tau)>100 \mathrm{GeV} / c \& P_{t}(l)>100$ $\mathrm{GeV} / c$. 


\subsection{Observed Limits}

We set limits in the context of two simplified models: 'simplified gravity' and 'simplified gauge' as described in Section 2.6.7. The final limit contours for the SUSY cross section are set in the $\mathrm{M}$ (Chargino) vs. $\mathrm{M}$ (Slepton) plane for certain chosen values of the LSP mass. An example of the signal points generated our the exclusion plane is shown in Figure 8.5. Limits are calculated at each signal point to then form a limit contour. To improve the $\mathrm{s} / \sqrt{\mathrm{b}}$ ratio we implement a $\mathbb{E}_{T}$ cut optimal for the kinematics of each signal point used in the exclusion plane. We define these event-level $E_{T}$ cuts in an analytic form so they can easily be determined by theorists to reinterpret the cross section limits. This allows us the flexibility to have different $\mathbb{E}_{T}$ cuts accommodating the different kinematics of various signal points, while preserving a simple interpretation of the resultant limits. To find the limits, the analysis is performed at the given $\mathbb{E}_{T}$ cut for the signal point, and limits are found for the expected signal and background. All systematic and statistical errors are incorporated in limit calculations.

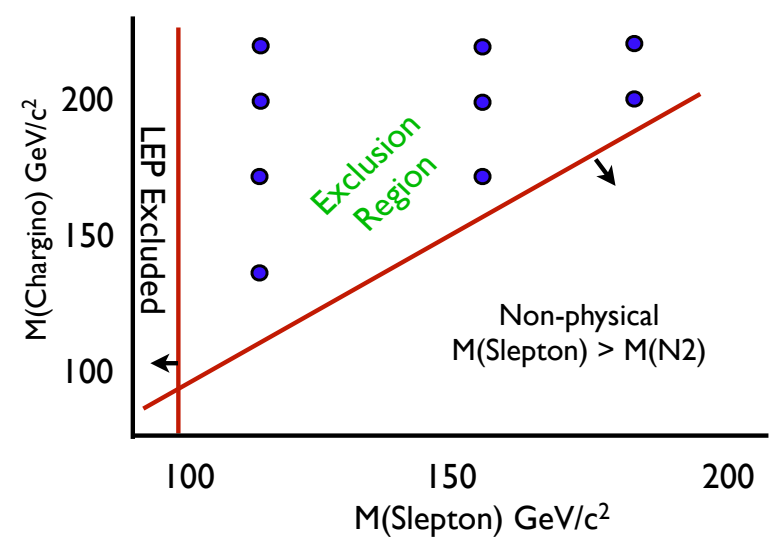

Figure 8.5: Schematic of the chosen signal points for the simplified gravity point with $M(L S P)=120 \mathrm{GeV} / c^{2}$.

\subsection{1 $\ddot{E}_{T}$ Cut Optimization}

In an effort to create a simple analytical expression for the best $\mathbb{E}_{T}$ cut at each signal points, we first scan over the point and find the optimal $s / \sqrt{b}$ in $10 \mathrm{GeV}$ steps from $E_{T}=0$ to $150 \mathrm{GeV}$. An example of such a scan is shown in Figure 8.6. For each chosen signal point point there is a maximum $s / \sqrt{b}$ as expected. We then correlate 
this best $\mathbb{E}_{T}$ cut with the parameters of the signal point.

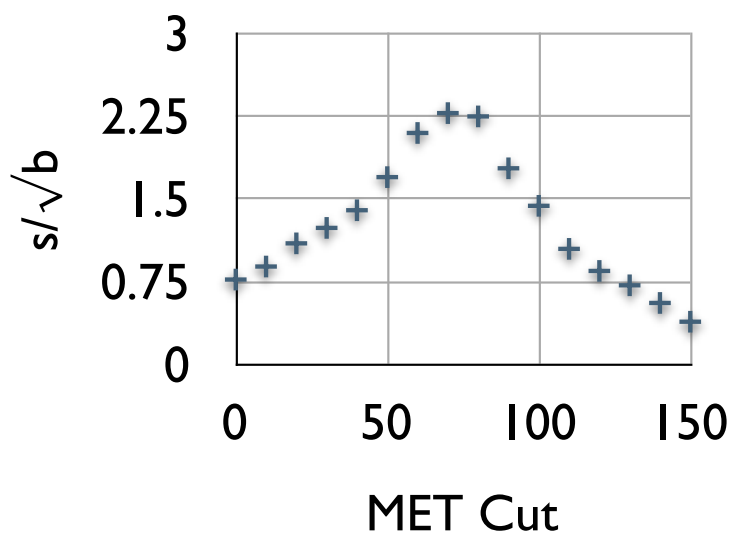

Figure 8.6: A scan of $s / \sqrt{b}$ for a 'simplified gravity' signal point with $M\left(\tilde{\chi}_{1}^{ \pm}\right)=220 \mathrm{GeV} / c^{2}, M(\tilde{l})=180 \mathrm{GeV} / c^{2}, M\left(\tilde{\chi}_{0}^{0}\right)=120 \mathrm{GeV} / c^{2}$.

A prevailing issue with chargino - neutralino searches is the abundance of $\mathbb{E}_{T}$ caused by the, single neutrino and two LSP particles in the decay chain. Leptonic tau decays add yet more $\mathbb{E}_{T}$ to the event. Because of the various uncorrelated angles of the particles that escape detection, there is no kinematic $\mathbb{E}_{T}$ cut that can be used to extract signal besides magnitude. To see how $\mathbb{E}_{T}$ magnitude might be correlated to signal points we go back to the Feynman diagram of our decays, as shown in Figure 8.7.

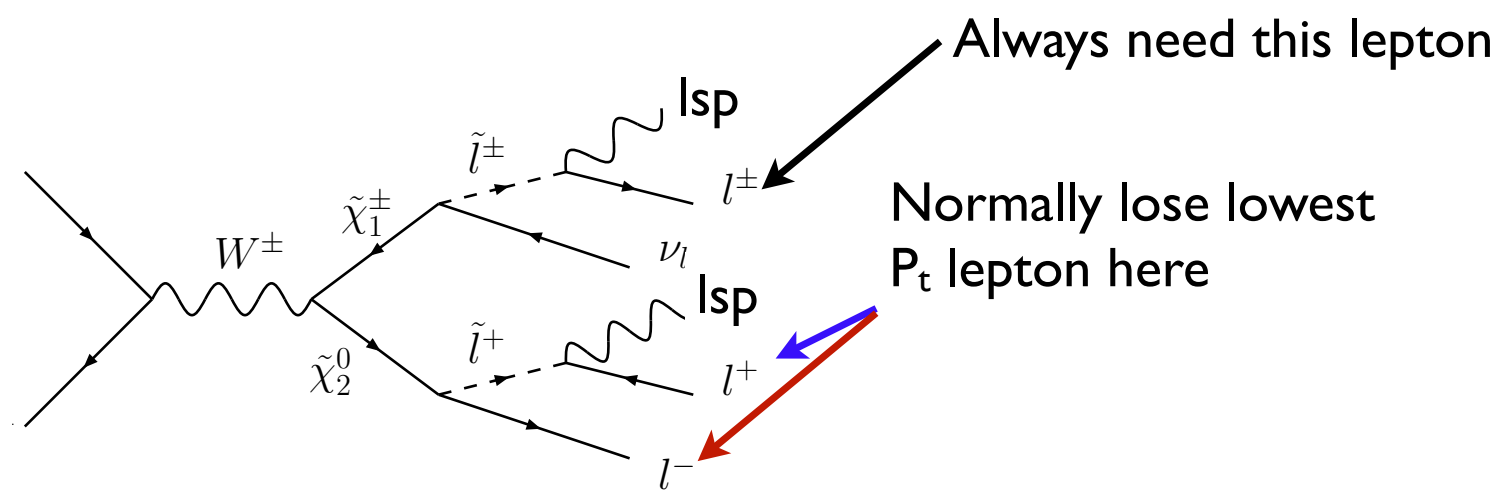

Figure 8.7: A Feynman diagram of our decays, to get a SS pair, we always need the top lepton coming from the chargino, and normally lose the lowest $P_{t}$ lepton coming from the neutralino.

We start with the assumption that the $\mathbb{E}_{T}$ will be correlated to the total energy in the event less the energy we miss. For the reasons described above, this is not a 
direct equivalence, just a correlated quantity. To get such a quantity we note that for a SS pair of leptons, we require detection of the lepton coming from the chargino. We then need the one same signed lepton coming from the neutralino, but we will normally detect the lepton with the highest $P_{t}$. Looking at the chargino branch then, we start with a generated $M\left(\tilde{\chi}_{1}^{ \pm}\right)$and do not detect the LSP or $M\left(\tilde{\chi}_{1}^{0}\right)$ for a correlated quantity that is then $M\left(\tilde{\chi}_{1}^{ \pm}\right)-M\left(\tilde{\chi}_{1}^{0}\right)$. For the neutralino branch of the decay, we again lose a LSP and we need to assume we detect the highest $P_{t}$ lepton. We either lose the lepton directly from the neutralino decay, in which case we lose roughly $\Delta M\left(\tilde{\chi}_{2}^{0}, \tilde{l}\right)$ or the lepton coming from the slepton, in which case the quantity lost is $\Delta M\left(\tilde{l}, \tilde{\chi}_{1}^{0}\right)$. We therefore have from the neutralino decays the quantity $M\left(\tilde{\chi}_{1}^{0}\right)-\operatorname{MIN}\left(\Delta M\left(\tilde{\chi}_{2}^{0}, \tilde{l}\right), M\left(\tilde{l}, \tilde{\chi}_{1}^{0}\right)\right)-M\left(\tilde{\chi}_{0}^{0}\right)$. Taking the two quantities together, and noting that in our model $M\left(\tilde{\chi}_{2}^{0}\right)=M\left(\tilde{\chi}_{1}^{ \pm}\right)$, we have:

$$
\mathbb{E}_{T} \approx 2 *\left(\mathrm{M}\left(\tilde{\chi}_{2}^{0}\right)-\mathrm{M}\left(\tilde{\chi}_{1}^{0}\right)\right)-\min \left(\Delta \mathrm{M}\left(\tilde{\chi}_{2}^{0}, \tilde{\mathrm{l}}\right), \Delta \mathrm{M}\left(\tilde{\mathrm{l}}, \tilde{\chi}_{1}^{0}\right)\right)
$$

This is the quantity we plot against the optimal $\mathbb{E}_{T}$ cuts we found in scanning $\mathrm{s} / \sqrt{\mathrm{b}}$ for each point. We have separate plots for each model and, for simplified gravity, each branching ratio used, for a total of three different $\mathbb{E}_{T}$ cut expressions, see Table 8.7. A sample of such a plot is shown in Figure 8.8 for the simplified gravity model and a branching ratio of $100 \%$. We fit a line to the points which becomes the functional form of our $\mathbb{E}_{T}$ cut.

The result is a fit to the data. Some points will not be cut at their optimal $s / \sqrt{b}$. For these points, none are suboptimal by more than about $20 \mathrm{GeV}$ which leads to a fairly insignificant effect on sensitivity, see Figure 8.6. We also see that there is a minimum at $\mathbb{E}_{T}=20 \mathrm{GeV}$ which appears due to the large backgrounds below that level in the $\mathbb{E}_{T}$ plots, as shown in Figure 8.3. The full set of cuts for all models are listed in Table 8.7.

\subsubsection{Limit Contours}

With optimal $\mathbb{E}_{T}$ cuts applied for every signal point generated, we can proceed to finding the expected limits. Again, we have a grid of points for each model and branching ratio to taus as seen in Figure 8.5. For the simplified gravity model we 


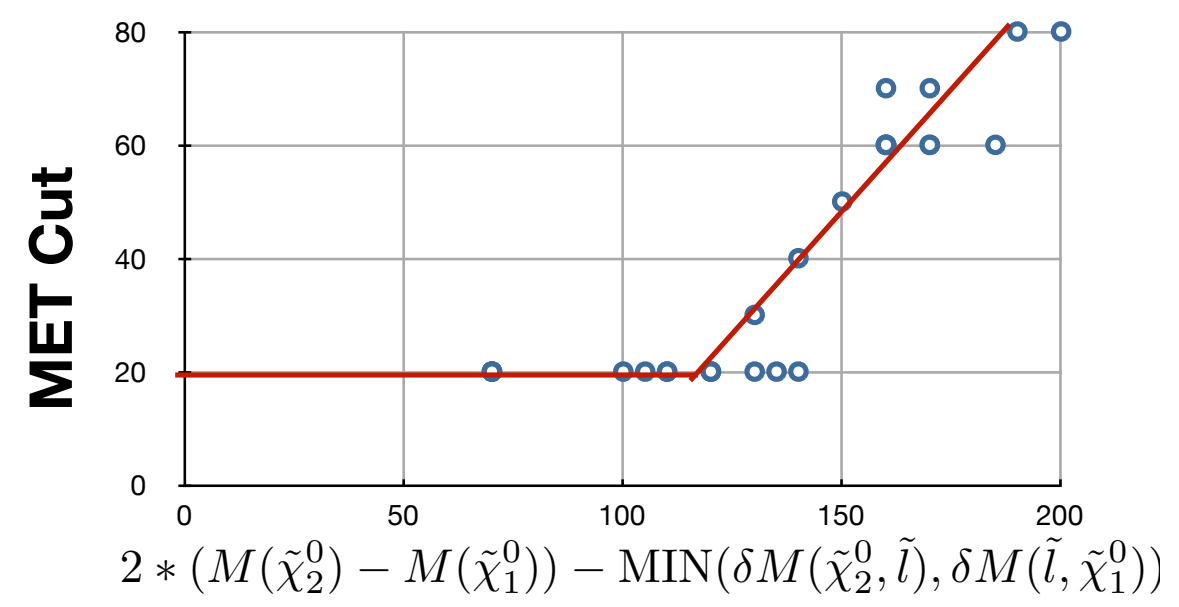

Figure 8.8: Best $\mathbb{E}_{T}$ cut versus our correlated quantity, Equation 8.1 for simplified gravity model with a branching ration to taus of $100 \%$. A red line shows the $\mathbb{E}_{T}$ cut functional form.

\begin{tabular}{|l|c|c|}
\hline \hline Model & BR to Taus $(\%)$ & $\mathscr{E}_{T} \mathrm{Cut}(\mathrm{GeV})$ \\
\hline Gauge & All Models & $\operatorname{MAX}(20, .20 * X+26)$ \\
\hline Gravity & 33 & $\operatorname{MAX}(20, .43 * X-4)$ \\
\hline Gravity & 100 & $\operatorname{MAX}(20, .85 * X-83)$ \\
\hline
\end{tabular}

Table 8.7: Functional forms for $\mathbb{E}_{T}$ cut for all models where $\mathrm{X}$ is defined in Equation 8.1.

have various LSP values as well. We apply the cuts in Table 8.7 at each point. We then get an expected background and expected signal. MCLimit [48] is used to find the expected limits at each signal point. We then use ROOT to interpolate limit contours of constant cross section limit at reasonable values for each model.

Below we show the expected limits for all models, branching ratios and LSP values. 


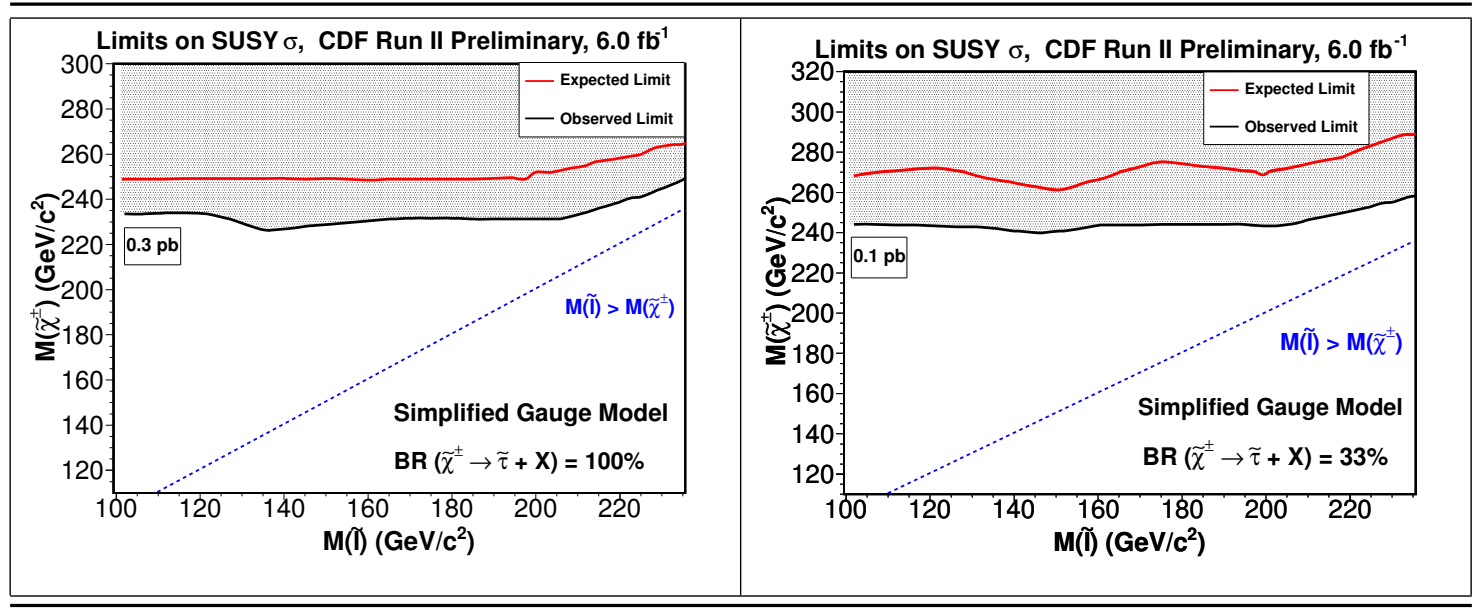

Figure 8.9: Expected limits (pb) for simplified gauge model for BR to taus of $100 \%$ (left), and $33 \%$ (right)

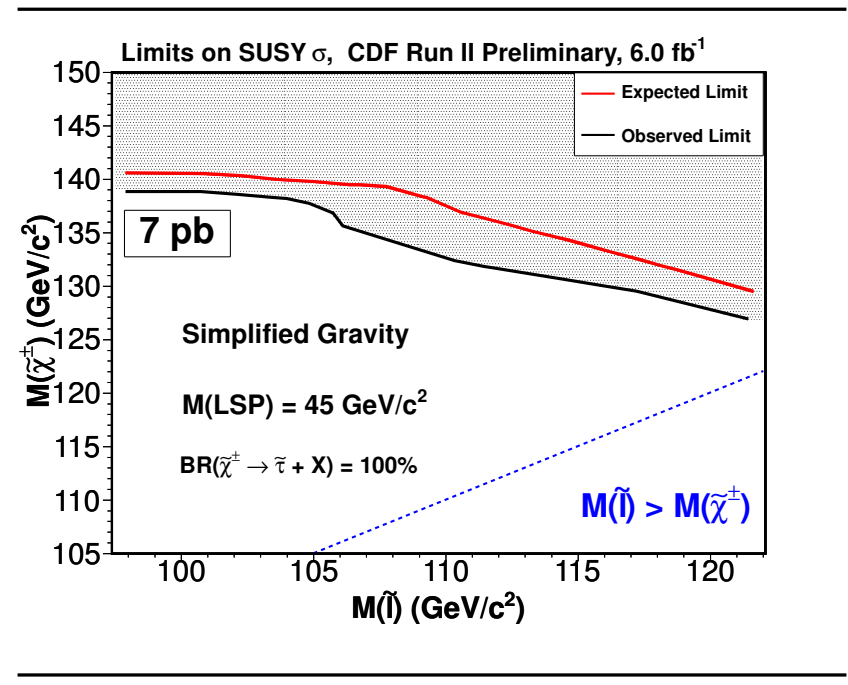

Figure 8.10: Expected limits (pb) for simplified gravity model with LSP = $45 \mathrm{GeV} / c^{2}$ for BR to taus of $100 \%$. 

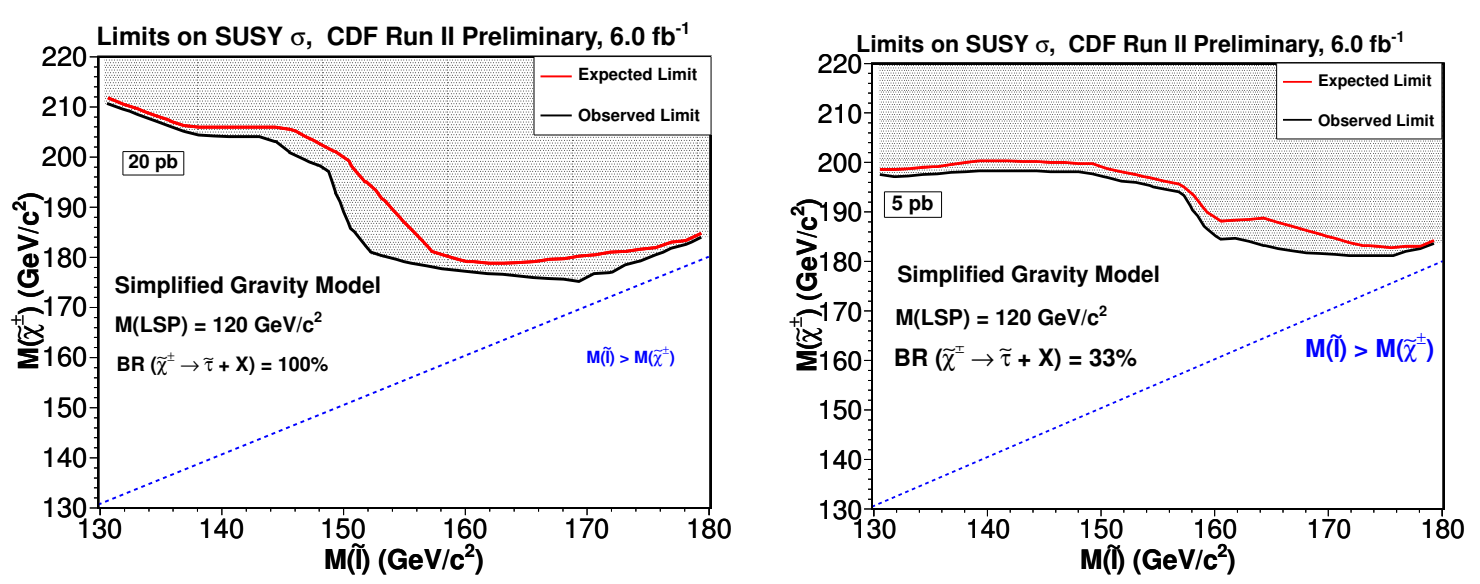

Figure 8.11: Expected limits (pb) for simplified gravity model with LSP = $120 \mathrm{GeV} / c^{2}$ for BR to taus of $100 \%$ (left), $33 \%$ (right).
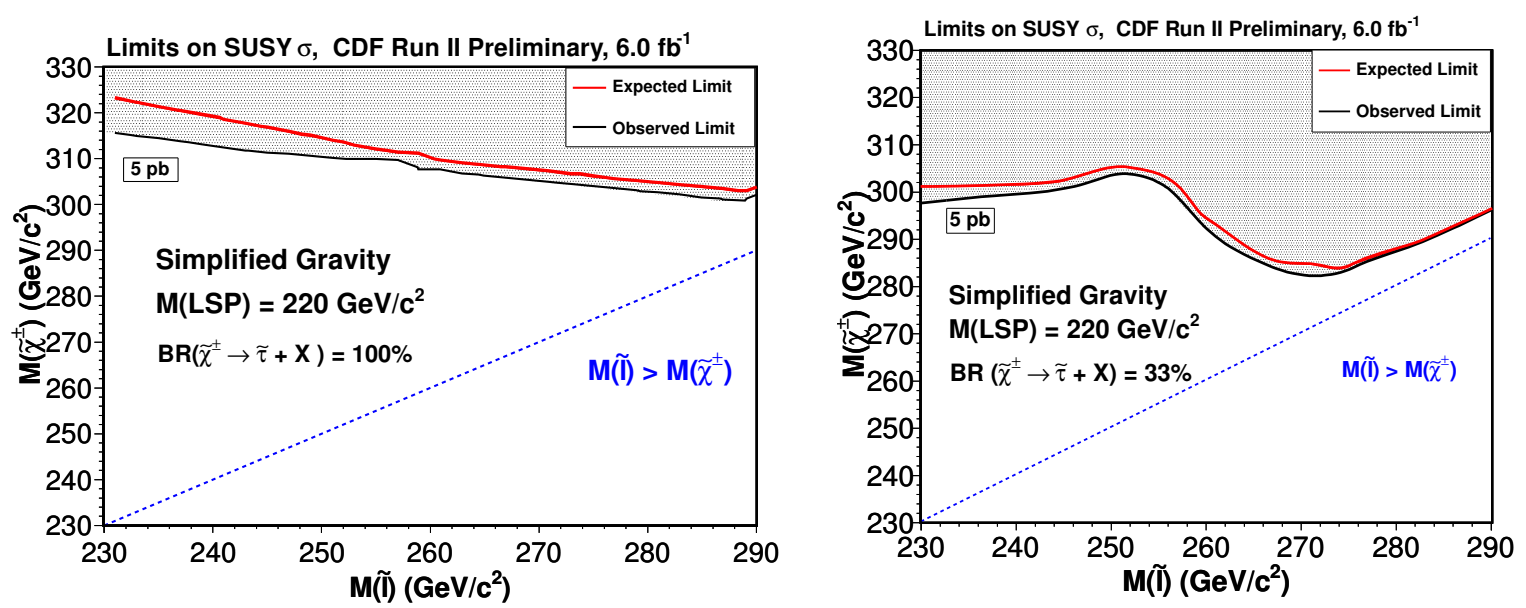

Figure 8.12: Expected limits (pb) for simplified gravity model with LSP = $220 \mathrm{GeV} / c^{2}$ for BR to taus of $100 \%$ (left), $33 \%$ (right). 


\section{Chapter 9}

\section{Conclusions}

We preformed a SUSY search for chargino-neutralino production by looking for an excess of same signed dilepton events over the background in $6.0 \mathrm{fb}^{-1}$ of data. We find no evidence of signal, and therefore set limits on SUSY production cross sections. We use simplified gauge and gravity models of SUSY breaking to improve the interpretation of our final limits for theorists.

The uniqueness of this analysis lies in several aspects of the way it is performed. Primarily, the inclusion of hadronic tau decays, as well as model parameters that force decays to taus, gives increased sensitivity to high $\tan \beta$ SUSY space. Additionally, we use a background model for fake taus that is almost totally data driven in the signal region. Finally, the use of generic simplified models frees us from being bound by particular models of SUSY.

This analysis is complimentary to similar analysis of same signed dileptons using only electrons and muons [49] and may potentially be combined with such analysis to increase sensitivity. Additionally, the results of this analysis may be used to limit other new physics models, such a a doubly charged Higgs boson [50].

We can improve on the results of this analysis in a few ways. The full CDF dataset will represent about $11.0 \mathrm{fb}^{-1}$ of data, expanding our sensitivity. We can also use additional parts of the CDF detectors which were non-fiducial to this analysis, dramatically adding to our sensitivity.

Ultimately, the LHC at CERN in Europe will have the last say on Supersymmetry. Already, the LHC limits on strong SUSY production channels exceed those from the 
Tevatron. Exclusion or discovery of electroweak modes of SUSY production will be close behind. Regardless, we will soon learn if the laws of nature contain Supersymmetry, and more importantly, what mysteries lie beyond the standard model. 


\section{Bibliography}

[1] W. M. Yao et al. Review of Particle Physics. J. Phys., G33:1-1232, 2006.

[2] David Griffiths. Introduction to Elementary Particles. Wiley, 1987.

[3] Jens Erler and Paul Langacker. Electroweak Model and Constraints on New Physics. 2004, hep-ph/0407097. Phys.Lett.B592:1(2004).

[4] David Griffiths. Introduction to Quantum Mechanics. Addison-Wesley, 2nd edition, 2005.

[5] R. Keith Ellis, W. James Stirling, and B. R. Webber. QCD and Collider Physics. Camb. Monogr. Part. Phys. Nucl. Phys. Cosmol., 8:1-435, 1996.

[6] M.E. Peskin and D.V. Schroeder. Introduction to Quantum Field Theory. Advanced Book Program. Addison-Wesley Pub. Co., 1995.

[7] Steven Weinberg. A Model of Leptons. Phys. Rev. Lett., 19:1264-1266, 1967.

[8] J.F. Gunion, H. Haber, G. Kane, and S. Dawson. The Higgs Hunter's Guide. Frontiers in physics. Perseus Publishing, 2000.

[9] G. Arnison et al. Experimental Observation of Lepton Pairs of Invariant Mass Around $95 \mathrm{GeV} / c^{2}$ at the CERN SPS collider. Phys. Lett., B126:398-410, 1983.

[10] G. Arnison et al. Experimental Observation of Isolated Large Transverse Energy Electrons with Associated Missing Energy at $\sqrt{s}=540-\mathrm{GeV}$. Phys. Lett., B122:103-116, 1983.

[11] Beat Jegerlehner, Frank Neubig, and Georg Raffelt. Neutrino Oscillations and the Supernova 1987a Signal. Phys. Rev. D, 54(1):1194-1203, Jul 1996. 
[12] Hinshaw, G. and Weiland, et al. Five-Year Wilkinson Microwave Anisotropy Probe Observations: Data Processing, Sky Maps, and Basic Results. 180:225245, February 2009, 0803.0732.

[13] Markevitch, M. and Gonzalez, A. H. and Clowe, D. et al. Direct Constraints on the Dark Matter Self-Interaction Cross Section from the Merging Galaxy Cluster 1E 0657-56. 606:819-824, May 2004, arXiv:astro-ph/0309303.

[14] Stephen P. Martin. A Supersymmetry Primer. 1997, hep-ph/9709356.

[15] S. Dawson. The MSSM and Why it Works. 1997, hep-ph/9712464.

[16] Leonard Susskind. Dynamics of Spontaneous Symmetry Breaking in the Weinberg- Salam Theory. Phys. Rev., D20:2619-2625, 1979.

[17] Glennys R. Farrar and Pierre Fayet. Phenomenology of the Production, Decay, and Detection of New Hadronic States Associated with Supersymmetry. Phys. Lett., B76:575-579, 1978.

[18] Lawrence J. Hall and Mahiko Suzuki. Explicit R-Parity Breaking in Supersymmetric Models. Nucl. Phys., B231:419, 1984.

[19] Hitoshi Murayama. Supersymmetry Phenomenology. 2000, hep-ph/0002232.

[20] R. Forrest. Search for Supersymmetry in $p \bar{p}$ Collisions at $\sqrt{s}=1.96 \mathrm{TeV}$ Using the Trilepton Signature of Chargino-Neutralino Production. 2009, 0910.1931.

[21] Joshua T. Ruderman and David Shih. Slepton co-NLSPs at the Tevatron. JHEP, 11:046, 2010, 1009.1665.

[22] Fermilab Beams Division. Website: http://www-bd.fnal.gov.

[23] Fermilab Beams Division. Run II Handbook. Website: http://wwwbd.fnal.gov/runII/index.html.

[24] R. Blair et al. The CDF-II Detector: Technical Design Report. Technical report, FNAL, 1996. FERMILAB-PUB-96/390-E.

[25] A. Sill. CDF Run II Silicon Tracking Projects. Nucl. Instrum. Meth., A447:1-8, 2000 .

[26] A. Allen Affolder et al. CDF Central Outer Tracker. Nucl. Instrum. Meth., A526:249-299, 2004. 
[27] L. Balka et al. The CDF Central Electromagnetic Calorimeter. Nucl. Instrum. Meth., A267:272, 1988.

[28] F. Abe et al. Measurement of the $\bar{p} p$ total cross-section at $\sqrt{s}=546 \mathrm{GeV}$ and 1800-GeV. Phys. Rev., D50:5550-5561, 1994.

[29] D. Acosta et al. The CDF Cherenkov Luminosity Monitor. Nucl. Instrum. Meth., A461:540-544, 2001.

[30] D. Acosta et al. The Performance of the CDF Luminosity Monitor. Nucl. Instrum. Meth., A494:57-62, 2002.

[31] The Trigger and Datasets Working Group. Run II Trigger Table and Datasets Plan. CDF/PHYS/TRIGGER/CDFR/4718, 2001.

[32] Torbjörn Sjöstrand, Stephen Mrenna, and Peter Z. Skands. PYTHIA 6.4 Physics and Manual. JHEP, 05:026, 2006, hep-ph/0603175.

[33] Johan Alwall, Michel Herquet, Fabio Maltoni, Olivier Mattelaer, and Tim Stelzer. MadGraph 5 : Going Beyond. JHEP, 1106:128, 2011, 1106.0522.

[34] Patrick Meade and Matthew Reece. BRIDGE: Branching Ratio Inquiry / Decay Generated Events. 2007, hep-ph/0703031.

[35] R. Brun et al. GEANT 3 Manual, CERN Program Library Long Writeup. 1994, W5013.

[36] H. L. Lai et al. Global QCD analysis of Parton Structure of the Nucleon: CTEQ5 Parton Distributions. Eur. Phys. J., C12:375-392, 2000, hep-ph/9903282.

[37] Sunil Somalwar Sourabh Dube, John Zhou. Medium ET Electron Identification Efficiency and Scale-Factors. CDF/8321, 2006.

[38] Ulysses Grundler Anyes Taffard Xiaojian Zhang. High-Pt Muons Recommended Cuts and Efficiencies for Summer 2006. CDF/8262, 2006.

[39] S. Baroiant, M. Chertok, M. Goncharov, et al. Energy Measurement for Hadronic Taus. CDF/6654, 2003.

[40] A. Anastassov, C. Cuenca, J. Conway, et al. Search for Neutral MSSM Higgs Bosons in the Tau Tau Decay Channel. CDF/8639, 2007. 
[41] A. Abulencia. Measurement of Sigma $p \bar{p} \rightarrow \mathrm{Z} . \mathrm{Br}(\mathrm{Z} \rightarrow 2 \tau)$ in $p \bar{p}$ Collisions at $\sqrt{s}=1.96 \mathrm{TeV}$. Phys. Rev., D75:092004, 2007.

[42] A. Abulencia et al. Search for Neutral MSSM Higgs Bosons Decaying to Tau Pairs in $p \bar{p}$ Collisions at $\sqrt{s}=1.96 \mathrm{TeV}$. Phys. Rev. Lett., 96:011802, 2006, hep-ex/0508051.

[43] Aidan Robson. Electroweak Cross-sections and Widths. 2008, 0810.2675.

[44] T. Aaltonen et al. Measurement of the ttbar Production Cross Section in ppbar Collisions at sqrt(s) $=1.96 \mathrm{TeV}$ Using Events with Large Missing Transverse Energy and Jets. 2011, 1105.1806.

[45] T. Aaltonen et al. Measurement of the $W W+W Z$ Production Cross Section Using a Matrix Element Technique in Lepton + Jets Events. Phys. Rev., D82:112001, 2010, 1008.4404.

[46] S. Jundariani et al. Luminosity Uncertainty for Run 2 up until August 2004. $\mathrm{CDF} / \mathrm{ANA} / 7446,2005$.

[47] A. Bhatti et al. Determination of the Jet Energy Scale at the Collider Detector at Fermilab. Nucl.Instrum.Meth., A566:375-412, 2006, hep-ex/0510047.

[48] T. Junk. Sensitivity, Exclusion and Discovery with Small Signals, Large Backgrounds, and Large Systematic Uncertainties. CDF/8128, 2007.

[49] T. Aaltonen et al. Search for New Physics in High $p_{T}$ Like-Sign Dilepton Events at CDF II. 2011, 1108.0101.

[50] A. G. Akeroyd, Cheng-Wei Chiang, and Naveen Gaur. Leptonic Signatures of Doubly Charged Higgs Boson Production at the LHC. JHEP, 11:005, 2010, 1009.2780. 


\section{List of Figures}

2.1 Particles of the standard model . . . . . . . . . . . . . . 6

2.2 Fundamental Feynman Diagram for QED. . . . . . . . . . . . 9

2.3 Corrections to the Higgs Mass - Fermion . . . . . . . . . . . . . . . . 14

2.4 Corrections to the Higgs Mass - Scalar . . . . . . . . . . . . . . . . 15

2.5 Chargino Neutralino Production mechanisms . . . . . . . . . . . 20

2.6 Diagrams of Neutralino Decay . . . . . . . . . . . . . . . . 22

2.7 Diagrams of Chargino Decay . . . . . . . . . . . . . . . . . 22

2.8 mSugra Exclusion Region . . . . . . . . . . . . . . . 23

3.1 Fermilab's acceleration chain. . . . . . . . . . . . . . . 27

3.2 Tevatron Collider Run II Integrated Luminosity . . . . . . . . . . . . 31

3.3 CDF Run II Detector Overview . . . . . . . . . . . . . . . . . 32

3.4 Wire planes on a COT endplate . . . . . . . . . . . . . 34

3.5 CDF Muon system coverage. . . . . . . . . . . . . . . 36

3.6 CDF Dead-timeless Trigger. . . . . . . . . . . . . . 38

3.7 CDF Trigger System Block Diagram. . . . . . . . . . . . . . 39 
4.1 XFT track finding efficiency for tracks that cross the $Z=0$ plane for two periods corresponding to category I and II triggers. We parameterized this efficiency as a function of the radius at which the tracks cross that plane, $R Z 0 . \ldots \ldots \ldots \ldots \ldots$

4.2 XFT track finding efficiency for tracks that cross the $Z=0$ plane wit SLAM confirmation (category IV triggers). We parameterized this efficiency as a function of the radius at which the tracks cross that plane, $R Z 0$. . . . . . . . . . . . . . . . .

4.3 XFT track finding efficiency for tracks that do not cross the $Z=0$ plane. We parameterized this efficiency as a function of the length of the track path in the $r-z$ plane. We measure for several different periods, here we show early data 3 layer tracks (left) as well as newer data from 4 layer tracks in the COT (right). . . . . . . . . . . .

4.4 We compare the efficiency in Jet20 data and QCD $18 \mathrm{MC}$ (top left) in order to obtain a correction scale factor (top right) that we then apply to $Z \rightarrow \tau \tau$ MC efficiency (bottom). . . . . . . . . . . .

4.5 L3 efficiency parameterized in terms of the number of primary vertices for the old trigger. . . . . . . . . . . . . . . . .

4.6 L3 efficiency parameterized in terms of the number of primary vertices for the new trigger. . . . . . . . . . . . . . . . . . . .

4.7 L3 "migration" efficiency for the Category I, II triggers, parameterized in terms of $\Delta \theta_{\text {min }} \ldots \ldots \ldots \ldots \ldots$

4.8 L3 "migration" efficiency for the Category III trigger, parameterized in terms of $\Delta \alpha_{m i n} \ldots \ldots \ldots \ldots \ldots \ldots$

4.9 Tau isolation and signal cones. . . . . . . . . . . . . . . .

5.1 Tau Relative Fake Rates. For simplicity, these particular fake rates are calculated with any jet in the event, for any number of tracks, and for all $\eta_{\text {det }}$. The used fake rates are parameterized as described in the text. 71

5.2 Fake Rate Verification. Conversion tagged electron events. Bands formed by leading and sub-leading jets in fake rate measurement. Data points are from tight tau identification. 
5.3 Fake Rate Verification. $W+$ Jet events with $W \rightarrow e \nu_{e}$. Bands formed by leading and sub-leading jets in fake rate measurement. Data points are from tight tau identification. . . . . . . . . . . . . .

5.4 Fake Rate Verification. $W+$ Jet events with $W \rightarrow \mu \nu_{\mu}$. Bands formed by leading and sub-leading jets in fake rate measurement. Data points are from tight tau identification. . . . . . . . . . . . . .

5.5 Fake Rate Verification. Non-isolated muons. Bands formed by leading and sub-leading jets in fake rate measurement. Data points are from tight tau identification. . . . . . . . . . . . . . .

5.6 Fake Rate Verification. Non-isolated electrons. Bands formed by leading and sub-leading jets in fake rate measurement. Data points are from tight tau identification. . . . . . . . . . . . . .

6.1 OS Control region plots. Electron channel (top) : Electron $E_{t}$ (left) and tau cluster $E_{T}$. Muon channel (bottom): Muon $P_{t}$ (left) and tau $P_{t}$ (right). . . . . . . . . . . . . . .

6.2 OS Control region plots. $\mathbb{E}_{T}$ linear scale (left) and log scale (right). .

6.3 OS Control region plots. Electron channel: Electron $H_{t}$ (top left) and tau $P_{T}$ (top right). Mass of e and $\mathbb{E}_{T}$ (middle left), transverse mass of e and $\mathscr{E}_{T}$ (middle right). Sum $P_{T}$ of the e and tau, (bottom left) and the transverse mass of the tau and the $\mathbb{E}_{T} . \ldots \ldots \ldots$

6.4 OS Control region plots. Muon channel: Muon, tau invariant mass (top left) and $d \phi\left(\mu, \mathbb{E}_{T}\right)$ (top right). Sum $P_{t}\left(\mu, \tau, \mathbb{E}_{T}\right)$ (middle left), Sum $P_{t}(\mu, \tau)$ (middle right). $E_{T}$ of the tau in both the siganl and iso cone, (bottom left) and the transverse mass of the muon and the $\mathbb{E}_{T}$.

8.1 SS signal region plots, Electron channel: Electron $E_{t}$ (top left) and log version (top right). With signal points (middle left) and log versions (middle right). Electron $H_{T}$ (bottom left) and the $\Sigma P_{T}(e, \tau)$ (bottom

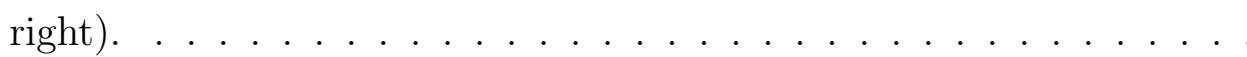


8.2 SS signal region plots, Muon channel: Muon $P_{t}$ (top left) and log version (top right). With signal points (middle left) and log versions (middle right). Tau Cluster $E_{T}$ (bottom left) and the $M_{T}\left(\mu, \mathbb{E}_{T}\right)$ (bottom right). . . . . . . . . . . . . . . .

8.3 SS signal region plots, $\mathbb{E}_{T}$ plots for both channels: $\mathbb{E}_{T}$ (top left) and log version (top right). With signal points (middle left) and log versions (middle right). Total background with total systematic errors (bottom left) and (bottom right). . . . . . . . . . . . . . . . 96

8.4 Distribution for Sub-region: $H_{t} \ldots \ldots \ldots$. . . . . . . . . . . 99

8.5 Schematic of the chosen signal points for the simplified gravity point with $M(L S P)=120 \mathrm{GeV} / c^{2}$. . . . . . . . . . . . . . . . . . 100

8.6 A scan of $s / \sqrt{b}$ for a 'simplified gravity' signal point with $M\left(\tilde{\chi}_{1}^{ \pm}\right)=$ $220 \mathrm{GeV} / c^{2}, M(\tilde{l})=180 \mathrm{GeV} / c^{2}, M\left(\tilde{\chi}_{0}^{0}\right)=120 \mathrm{GeV} / c^{2} \ldots \ldots 101$

8.7 A Feynman diagram of our decays, to get a SS pair, we always need the top lepton coming from the chargino, and normally lose the lowest $P_{t}$ lepton coming from the neutralino. . . . . . . . . . . . . . 101

8.8 Best $E_{T}$ cut versus our correlated quantity, Equation 8.1 for simplified gravity model with a branching ration to taus of $100 \%$. A red line shows the $\mathbb{E}_{T}$ cut functional form. . . . . . . . . . . . . 103

8.9 Expected limits (pb) for simplified gauge model for BR to taus of $100 \%$ (left), and 33\% (right) . . . . . . . . . . . . . . 104

8.10 Expected limits ( $\mathrm{pb}$ ) for simplified gravity model with LSP $=45 \mathrm{GeV} / c^{2}$ for BR to taus of $100 \%$. . . . . . . . . . . . . . . . 104

8.11 Expected limits (pb) for simplified gravity model with LSP $=120$ $\mathrm{GeV} / c^{2}$ for BR to taus of $100 \%$ (left), 33\% (right). . . . . . . . . 105

8.12 Expected limits (pb) for simplified gravity model with LSP $=220$ $\mathrm{GeV} / c^{2}$ for BR to taus of $100 \%$ (left), $33 \%$ (right). 


\section{List of Tables}

2.1 Fermionic sector of the standard model, antiparticles have opposite charge. . . . . . . . . . . . . . . . . . 4

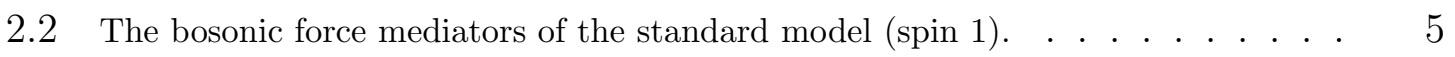

2.3 Chiral supermultiplets of the MSSM. . . . . . . . . . . . . 17

2.4 Gauge supermultiplets of the MSSM. . . . . . . . . . . . . . . . . 17

3.1 Tevatron parameters for Run II. . . . . . . . . . . . . . . . . . . 30

4.1 "Lepton plus track" trigger paths used in this analysis, grouped by common trigger features or categories. . . . . . . . . . . . . 45

4.2 L2 cluster cuts. . . . . . . . . . . . . . . . . . . . . . . 46

4.3 L3 Isolation annulus definition, and cuts for isolation tracks . . . . . 46

4.4 Fitted parameters for the L2 trigger efficiency as a function of $R Z 0$ (see Sub-Section 4.2.2) for tracks that cross $Z=0$. . . . . . . . . . . 50

4.5 Fitted parameters for the L2 trigger efficiency as a function of $\operatorname{Lr} z$ for 3 (left) and 4 (right) Layer tracks that do not cross $z=0 . \quad$. . . . . 52

4.6 Results of the fits to $\Delta \theta_{\text {min }}$ and $\Delta \alpha_{\text {min }} \ldots \ldots \ldots$. . . . . . . 55

6.1 OS electron channel control region. . . . . . . . . . . . . . . . . . . 82

6.2 OS muon channel control region. . . . . . . . . . . . . . . 83

6.3 Total OS control region. . . . . . . . . . . . . 83 
7.1 A summary of all systematic uncertainties used in the analysis, what they are applied to, and their value $(\%)$. . . . . . . . . . . . 90

8.1 SS signal region used in limit setting, $\mathbb{E}_{T}>20 \mathrm{GeV}$. Both e and $\mu$

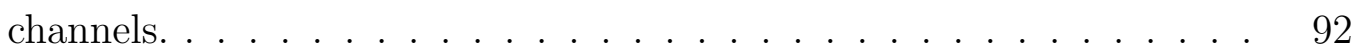

8.2 SS signal region used in limit setting, $\mathbb{E}_{T}>20 \mathrm{GeV}$. Electron Channel. 92

8.3 SS signal region used in limit setting, $\mathbb{E}_{T}>20 \mathrm{GeV}$. Muon Channel. 93

8.4 Prediction for Sub-region: $M(\tau, l)>200 \mathrm{GeV} / c^{2} \ldots \ldots . . \ldots 98$

8.5 Prediction for Sub-region: $H_{t}>300 \mathrm{GeV} / c \ldots \ldots . \ldots 9$

8.6 Prediction for Sub-region: $P_{t}(\tau)>100 \mathrm{GeV} / c \& P_{t}(l)>100 \mathrm{GeV} / c . . \quad 99$

8.7 Functional forms for $\mathbb{E}_{T}$ cut for all models where $\mathrm{X}$ is defined in Equation 8.1. . . . . . . . . . . . . . . . . . . . 103 


\section{Glossary}

COT Central Outer Tracker

L00 Layer 00

SVXII Silicon Vertex Detector

ISL Intermediate Silicon Layer

CEM Central Electromagnetic Calorimeter

CHA Central Hadronic Calorimeter

WHA Endwall Hadron Calorimeter

CES Shower Maximum Detector

PEM Electromagnetic Plug Calorimeter

PHA Hadronic Plug Calorimeter

PES Plug Shower Maximum

CPR Central Preradiator

PPR Plug Preradiator

CMU Central Muon Detector

CMP Central Muon Upgrade Detector

CMX Central Muon Extension Detector

IMU Intermediate Muon Detector

BMU Barrel Chambers 
CSP Central Muon Scintillator Upgrade

CSX Central Muon Extension Scintillator

BSU Barrel Scintillator Upgrade

TSU Toroid Scintillator Upgrade

CLC Čerenkov Luminosity Counters

XFT eXtremely Fast Tracker

XTRP Extrapolation Module

XCES Shower Maximum Locator

SVT Silicon Vertex Tracker 